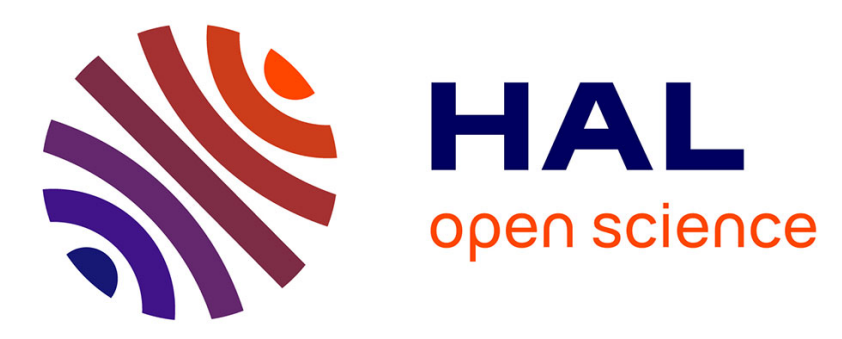

\title{
Multiscale modelling of complex fluids: A mathematical initiation.
}

Claude Le Bris, Tony Lelièvre

\section{To cite this version:}

Claude Le Bris, Tony Lelièvre. Multiscale modelling of complex fluids: A mathematical initiation.. [Research Report] RR-6275, INRIA. 2007. inria-00165171v3

\section{HAL Id: inria-00165171 https://hal.inria.fr/inria-00165171v3}

Submitted on 31 Aug 2007

HAL is a multi-disciplinary open access archive for the deposit and dissemination of scientific research documents, whether they are published or not. The documents may come from teaching and research institutions in France or abroad, or from public or private research centers.
L'archive ouverte pluridisciplinaire HAL, est destinée au dépôt et à la diffusion de documents scientifiques de niveau recherche, publiés ou non, émanant des établissements d'enseignement et de recherche français ou étrangers, des laboratoires publics ou privés. 
INSTITUT NATIONAL DE RECHERCHE EN INFORMATIQUE ET EN AUTOMATIQUE

\title{
Multiscale modelling of complex fluids: A mathematical initiation.
}

\author{
C. Le Bris - T. Lelièvre
}

$\mathbf{N}^{\circ} 6275$

Août 2007

Thème NUM 



\title{
Multiscale modelling of complex fluids: A mathematical initiation.
}

\author{
C. Le Bris 田, T. Lelièvre * \\ Thème NUM — Systèmes numériques \\ Projets MicMac \\ Rapport de recherche $\mathrm{n}^{\circ} 6275$ - Août 2007 -99 pages
}

\begin{abstract}
We present a general introduction to the multiscale modelling and simulation of complex fluids. The perspective is mathematical. The level is elementary. For illustration purposes, we choose the context of incompressible flows of infinitely dilute solutions of flexible polymers, only briefly mentioning some other types of complex fluids. We describe the modelling steps, compare the multiscale approach and the purely macroscopic, more traditional, approach. We also introduce the reader with the appropriate mathematical and numerical tools. A complete set of codes for the numerical simulation is provided, in the simple situation of a Couette flow. This serves as a test-bed for the numerical strategies described in a more general context throughout the text. A dedicated section of our article addresses the mathematical challenges on the front of research.
\end{abstract}

Key-words: non-Newtonian flows, complex fluids, polymer flow, multiscale modelling, Couette flow, Hookean and FENE dumbbell models, Oldroyd-B model, Fokker-Planck equation, stochastic differential equation

\footnotetext{
* CERMICS, École Nationale des Ponts et Chaussées, 6 \& 8, avenue Blaise Pascal, 77455 Marne-LaVallée Cedex 2, and INRIA Rocquencourt, MICMAC project-team, Domaine de Voluceau, B.P. 105, 78153 Le Chesnay Cedex, FRANCE, e-mail: \{lebris, lelievre\}@cermics.enpc.fr
} 


\section{Modèles multiéchelles pour les fluides complexes: Une initiation mathématique.}

Résumé : Ce document est une introduction à la modélisation et la simulation multiéchelles des fluides complexes, d'un point de vue mathématique, en restant à un niveau élémentaire. Nous nous concentrons principalement sur les écoulements incompressibles de solutions diluées de polymères flexibles, et nous mentionnons plus brièvement des modèles multi-échelles pour d'autres fluides complexes. Nous décrivons les différentes étapes de la modélisation, en comparant l'approche multi-échelles et l'approche purement macroscopique, plus traditionnelle. Les outils mathématiques et numériques nécessaires sont introduits. Un ensemble de codes Matlab pour la simulation numérique d'un écoulement de Couette est fourni. Ces codes illustrent les stratégies numériques décrites dans un cadre plus général au cours du texte. Une section est dédiée aux aspects les plus récents de la recherche en mathématiques appliquées sur le thème des fluides complexes en général, et en particulier sur les modèles multi-échelles pour les fluides complexes.

Mots-clés : Fluides non-newtoniens, fluides complexes, fluides polymériques, modélisation multi-échelles, écoulement de Couette, modèles des dumbbells hookéens et FENE, modèle Oldroyd-B, équation de Fokker-Planck, équation différentielle stochastique 


\section{Contents}

1 Introduction 4

2 Newtonian and non-Newtonian fluids 6

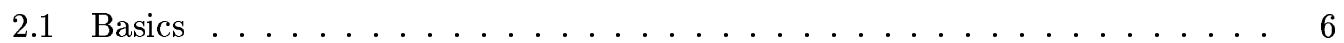

2.2 Non-Newtonian fluids . . . . . . . . . . . . . . . . . . 7

2.2.1 Some experimental observations . . . . . . . . . . . . 7

2.2.2 Modelling of non-Newtonian fluids . . . . . . . . . . . . . . . 10

2.3 Some macroscopic models for viscoelastic fluids . . . . . . . . . . . . . . . 12

2.3.1 More on differential models . . . . . . . . . . . . . . . . . 12

2.3 .2 More on integral models . . . . . . . . . . . . . . . . . . 15

\begin{tabular}{|lll}
3 & Multiscale modelling of polymeric fluids & 17
\end{tabular}

3.1 Statistical mechanics of the free chain . . . . . . . . . . . . . . . . 20

3.1.1 Generalities . . . . . . . . . . . . . . . . . . . 20

3.1 .2 The Hookean model . . . . . . . . . . . . . . . . . . . . 22

3.2 The multiscale model . . . . . . . . . . . . . . . . . . . . . 23

$\begin{array}{lll}4 & \text { The stochastic approach } & 28\end{array}$

4.1 Initiation to Stochastic Differential Equations . . . . . . . . . . . . . . . 28

4.1.1 The Monte Carlo method . . . . . . . . . . . . . . . . . . . . 28

4.1.2 Brownian motion and simple stochastic differential equations . . . . . 29

4.1 .3 Stochastic integration . . . . . . . . . . . . . . . . . 31

4.1 .4 Itô calculus and Fokker Planck equation . . . . . . . . . . . . 32

4.1 .5 Discretization of SDEs . . . . . . . . . . . . . . . . 35

4.2 Back to the modelling . . . . . . . . . . . . . . . . . . 37

4.2 .1 The microscopic equation of motion . . . . . . . . . . . . . 37

4.2 .2 The stress tensor . . . . . . . . . . . . . . . . . . 40

4.2 .3 The force . . . . . . . . . . . . . . . . . . . . . . 41

4.3 The multiscale model . . . . . . . . . . . . . . . . . . . . . 44

4.4 Schematic overview of the simulation . . . . . . . . . . . . . . 46

4.4.1 Numerical methods . . . . . . . . . . . . . . . . . . . . . . . . 46

4.4 .2 Main difficulties . . . . . . . . . . . . . . . . . . 4 47

4.5 Upsides and downsides of multiscale modelling for complex fluids . . . . . . . 48

4.5.1 Micro-macro vs macro-macro modelling . . . . . . . . . . . . . . 48

4.5 .2 Fokker-Planck vs SDE formulation . . . . . . . . . . . . . . . . . 49

5 Numerical simulation of a test case $\quad 51$

5.1 Setting of the problem . . . . . . . . . . . . . . . . . . 51

5.2 Discretization of the macroscopic equation . . . . . . . . . . . 53

5.3 Microscopic problem: the deterministic approach . . . . . . . . . . . 55

5.4 Microscopic problem: the stochastic approach . . . . . . . . . . . . 56 
5.5 Extension to the FENE model . . . . . . . . . . . . . . . 60

5.6 Matlab codes . . . . . . . . . . . . . . . . . . 61

6 Mathematical and numerical issues $\quad 69$

6.1 Overview of the main difficulties . . . . . . . . . . . . . . . . . . . 69

6.2 Macroscopic models . . . . . . . . . . . . . . . . . . . . . . . . . . . . . . . . . . 70

6.2.1 Mathematical results . . . . . . . . . . . . . . . . 70

6.2 .2 Numerical methods . . . . . . . . . . . . . . . . 71

6.3 Multiscale models ...................... . . 73

6.3.1 Simplifications of the equations . . . . . . . . . . . . . 74

6.3.2 Mathematical Analvsis . . . . . . . . . . . . . . . . 75

6.3.3 Numerical methods . . . . . . . . . . . . . . . 81

7 Other types of complex fluids $\quad 84$

7.1 Liquid crvstals . . . . . . . . . . . . . . . . . . 84

7.2 Suspensions . . . . . . . . . . . . . . . . . . . . . 86

7.3 Blood flows . . . . . . . . . . . . . . . . . . 87

\section{Introduction}

This article presents a general introduction to the multiscale modelling and simulation of complex fluids. The perspective is mathematical. The level is elementary. For illustration purposes, we choose the context of incompressible flows of infinitely dilute solutions of flexible polymers. This category of fluids is that for which the mathematical understanding is the most comprehensive one to date. It is therefore an adequate prototypical context for explaining the recently developed multiscale approach for the modelling of complex fluids, and more precisely for that of fluids with microstructures. Other types of complex fluids, also with microstructures, such as liquid crystals, suspensions, blood, may also be modeled by such types of models. However the modelling is either less understood mathematically, or more intricate and technical to describe (or both). The former case is therefore more appropriate for an initiation.

We describe the modelling steps, compare the multiscale approach and the purely macroscopic, more traditional, approach. We also introduce the reader to the appropriate mathematical and numerical tools.

The readership we wish to reach with our text consists of two categories, and our purpose is thus twofold.

Our primary purpose is to describe to mathematics (or applied mathematics) students, typically at undergraduate level, or in their early years of graduate studies, the various steps involved in a modern modelling endeavor. The multiscale simulation of complex fluids is an excellent example for this. Thinking to this audience, we concentrate ourselves on key issues in the modelling, assuming only the knowledge of some basic notions of continuum mechanics (briefly recalled in Section 2) and elaborating on those in Section 3 to construct the simplest 
multiscale models for complex fluids. We also assume that these students are familiar with some standard notions about partial differential equations and the discretization techniques commonly used for their simulation. On the other hand, because we know from our teaching experience that such students often have only a limited knowledge in probability theory and stochastic analysis, we choose to give (in Section 4) a crash course on the elements of stochastic analysis needed to manipulate the stochastic versions of the models for complex fluids. The latter are introduced in the second part of Section 4 To illustrate the notions introduced on a very simple case, and to allow our readers to get into the heart in the matter, we devote the entire Section [5 to several possible variants of numerical approaches for the simulation of start-up Couette flows. This simple illustrative case serves as a test-bed for the numerical strategies described in a more general context throughout the text. A complete set of codes for the numerical simulation is provided, which we encourage the readers to work with like in a hands-on session.

The second category of readers we would like to get interested in the present article consists of practitioners of the field, namely experts in complex fluids rheology and mechanics, or chemical engineers. The present text could serve, we believe, as an introduction to a mathematical viewpoint on their activity. Clearly, the issues we, as mathematicians and computational scientists, emphasize, are somewhat different from those they consider on a regular basis. The perspective also is different. We are looking forward to their feedback on the text.

For both communities above, we are aware that an introductory text, although useful, is not fully satisfactory. This is the reason why we devote a section of our article, Section 6 . to a short, however comprehensive, description of the mathematical and numerical challenges of the field. This section is clearly more technical, and more mathematical in nature, than the preceding ones. It is, hopefully, interesting for advanced graduate students and researchers, professionals in mathematics, applied mathematics or scientific computing. The other readers are of course welcome to discover there what the exciting unsolved questions of the field are.

Finally, because we do not want our readers to believe that the modelling of infinitely dilute solutions of flexible polymers is the only context within complex fluids science where mathematics and multiscale simulation can bring a lot, we close the loop, describing in our last Section 7 some other types of complex fluids where the same multiscale approach can be employed. 


\section{Incompressible fluid mechanics: Newtonian and non- Newtonian fluids}

\subsection{Basics}

To begin with, we recall here some basic elements on the modelling of incompressible fluids.

Consider a viscous fluid with volumic mass (or density) $\rho$, flowing at the velocity $\boldsymbol{u}$. It experiences external forces $\boldsymbol{f}$ per unit mass. Denote by $\boldsymbol{T}$ the stress tensor.

The equation of conservation of mass for this fluid reads

$$
\frac{\partial \rho}{\partial t}+\operatorname{div}(\rho \boldsymbol{u})=0
$$

On the other hand, the equation expressing the conservation of momentum is

$$
\frac{\partial(\rho \boldsymbol{u})}{\partial t}+\operatorname{div}(\rho \boldsymbol{u} \otimes \boldsymbol{u})-\operatorname{div} \boldsymbol{T}=\rho \boldsymbol{f}
$$

For such a viscous fluid, the stress tensor reads

$$
\boldsymbol{T}=-p I d+\tau,
$$

where $p$ is the (hydrodynamic) pressure, and $\tau$ is the tensor of viscous stresses. In order to close the above set of equations, a constitutive law (or constitutive relation) is needed, which relates the viscous stress $\boldsymbol{\tau}$ and the velocity field $\boldsymbol{u}$, namely

$$
\boldsymbol{\tau}=\boldsymbol{\tau}(\boldsymbol{u}, \rho, \ldots) .
$$

Note that (2.4) is symbolic. A more precise formulation could involve derivatives in time, or in space, of the various fields $\boldsymbol{\tau}, \boldsymbol{u}, \rho, \ldots$

Assuming that $\boldsymbol{\tau}$ linearly depends on $\boldsymbol{u}$, that $\boldsymbol{\tau}$ is invariant under the change of Galilean referential, and that the fluid has isotropic physical properties, it may be shown that the relation between $\boldsymbol{\tau}$ and $\boldsymbol{u}$ necessarily takes the following form

$$
\boldsymbol{\tau}=\lambda(\operatorname{div} \boldsymbol{u}) I d+2 \eta \boldsymbol{d}
$$

where $\lambda$ and $\eta$ are two scalar coefficients (called the Lamé coefficients). The latter depend, in full generality, of the density $\rho$ and the temperature. In (2.5), $d$ denotes the (linearized) rate of deformation tensor (or rate of strain tensor)

$$
\boldsymbol{d}=\frac{1}{2}\left(\nabla \boldsymbol{u}+\nabla \boldsymbol{u}^{T}\right)
$$

When a fluid obeys the above assumptions, it is called a Newtonian fluid. The kinetic theory of gases allows to show that

$$
\lambda=-\frac{2}{3} \eta
$$


and the common practice is to consider both coefficients $\lambda$ and $\eta$ constant.

The system of equations (2.1)-(2.2)-(2.3)-(2.5)-(2.6)-(2.7) allows then to describe the motion of the fluid. When accounting for temperature effects, or for compressible effects, the system is complemented by two additional equations, the energy equation and an equation of state (relating $p, \rho$ and $T$ ). We will neglect such effects in the following and assume the temperature is constant and the fluid is incompressible:

$$
\operatorname{div} \boldsymbol{u}=0 .
$$

Then, equations (2.1)-(2.2)-(2.3)-(2.5)-(2.6)-(2.7)-(2.8) provide the complete description of the evolution of the Newtonian fluid.

Let us additionally assume the fluid has constant density

$$
\rho=\rho_{0} .
$$

Such a fluid is often called homogeneous. The equation of conservation of momentum then rewrites

$$
\rho\left(\frac{\partial \boldsymbol{u}}{\partial t}+(\boldsymbol{u} \cdot \nabla) \boldsymbol{u}\right)-\eta \Delta \boldsymbol{u}+\nabla p=\rho \boldsymbol{f}
$$

It is supplied with the divergence-free condition

$$
\operatorname{div} \boldsymbol{u}=0 .
$$

The couple of equations (2.9)-(2.10) form is the celebrated Navier-Stokes equation for the motion of incompressible homogeneous viscous Newtonian fluids.

\subsection{Non-Newtonian fluids}

\subsubsection{Some experimental observations}

Non-Newtonian fluids, and, in particular, viscoelastic fluids are ubiquitous in industry (oil industry, food industry, rubber industry, for example), as well as in nature (blood is a viscoelastic fluid). As mentioned above, Newtonian fluids are characterized by the fact that the stress is proportional to the rate of deformation $\frac{1}{2}\left(\nabla \boldsymbol{u}+\nabla \boldsymbol{u}^{T}\right)$ : this is viscosity. For elastic solids, the stress is proportional to the deformation (see the tensors (2.35) $C_{t}$ or (2.36) $\boldsymbol{F}_{t}$ below for some measure of deformation): this is elasticity. The characteristic feature of viscoelastic fluids is that their behavior is both viscous and elastic. Polymeric fluids are one instance of viscoelastic fluids.

To explore the rheological behavior of viscoelastic fluids (rheology is the science studying why and how fluids flow), physicists study their response to so-called simple flows (typically flows in pipes or between two cylinders) to obtain so-called material functions (such as shear viscosity, differences of normal stress, see below). Typically, for such flows, the velocity field is known and is not influenced by the non-Newtonian features of the fluid. This owes to the 
fact that the velocity field is homogeneous, which means that $\nabla \boldsymbol{u}$ does not depend on the space variable. Such flows are called homogeneous flows. Two types of simple flows are very often used in practice: simple shear flows and elongational flows (see R.B. Bird, R.C. Armstrong and O. Hassager [11, Chapter 3]). We focus here on simple shear flows. In practical situations (in an industrial context for example), flows are generally more complicated than the simple flows used to characterize the rheological properties of the fluids: such flows are called complex flows. Complex flows are typically not homogeneous: $\nabla \boldsymbol{u}$ depends on the space variable $\boldsymbol{x}$.
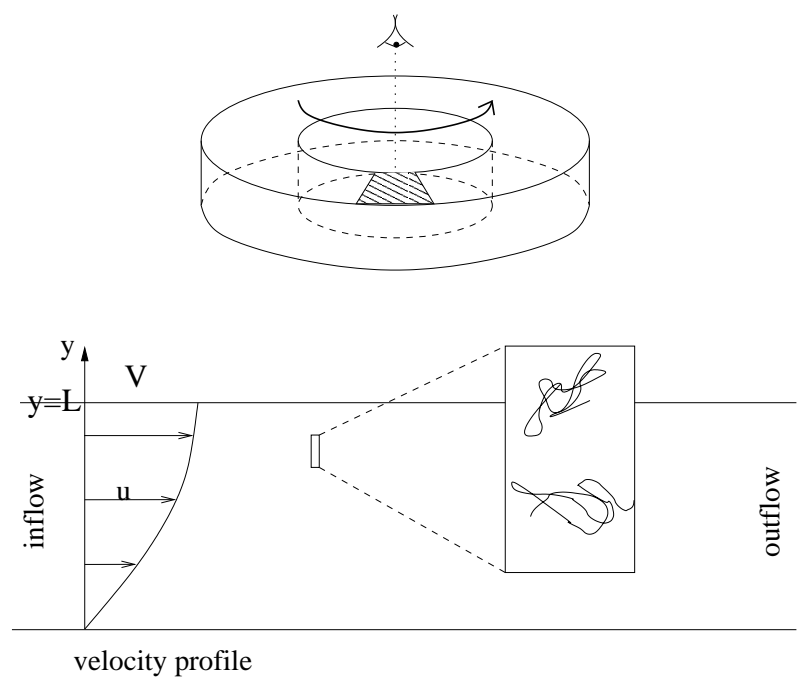

Figure 1: Schematic representation of a rheometer. On an infinitesimal angular portion, seen from the top, the flow is a simple shear flow (Couette flow) confined between two planes with velocity profile $(u(t, y), 0,0)$.

In a simple shear flow, the velocity $\boldsymbol{u}$ has the following form:

$$
\boldsymbol{u}(t, \boldsymbol{x})=(\dot{\gamma}(t) y, 0,0),
$$

where $\boldsymbol{x}=(x, y, z)$ and $\dot{\gamma}$ is the shear rate. The shear viscosity $\eta$ :

$$
\eta(t)=\frac{\boldsymbol{\tau}_{x, y}(t)}{\dot{\gamma}(t)},
$$

and the first and second differences of normal stress:

$$
\begin{aligned}
& N_{1}(t)=\boldsymbol{\tau}_{x, x}(t)-\boldsymbol{\tau}_{y, y}(t), \\
& N_{2}(t)=\boldsymbol{\tau}_{y, y}(t)-\boldsymbol{\tau}_{z, z}(t),
\end{aligned}
$$



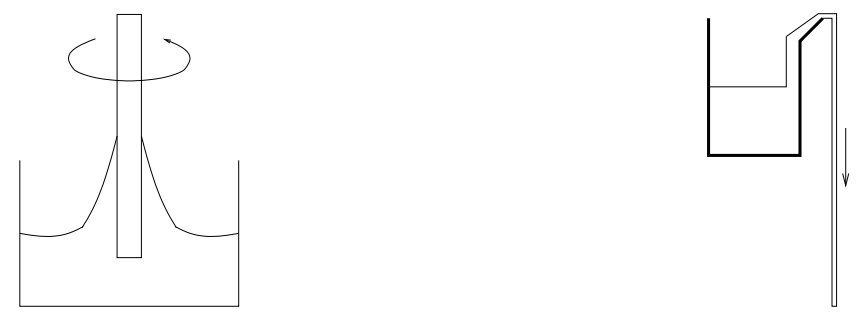

Figure 2: Schematic representation of two unexpected, counterintuitive behaviors for some polymeric fluids: the rod-climbing effect (left) and the open syphon effect (right).

may be measured experimentally. For Newtonian fluids, the shear viscosity is constant, and both $N_{1}$ and $N_{2}$ vanish. This is not the case in general for viscoelastic fluids. In particular, for many non-Newtonian fluids, $\eta$ is a decreasing function of $\dot{\gamma}$ (this property is called shearthinning), goes to a constant $\eta_{\infty}$ when $\dot{\gamma}$ goes to infinity, and goes to some value $\eta_{0}$ (the zero-shear rate viscosity) when $\dot{\gamma}$ goes to zero.

In practice, such flows are studied in rheometers, the fluid being confined between two cylinders. The outer cylinder is fixed, the inner one is rotating (see Figure 1). On an infinitesimal portion, the flow can be approximated by a simple shear flow. We will return to this in Section $[5$

The simple shear flow may also be useful to study the dynamics of the fluid using an oscillating excitation: $\dot{\gamma}(t)=\gamma_{0} \cos (\omega t)$. The in phase response with the deformation is related to the elasticity of the fluid. The out of phase response is related to the viscosity of the fluid. This can be easily understood for example on the simple Maxwell model presented below, and an analogy with electric circuits (see Figure 4 ).

Before addressing the modelling in details, let us mention some peculiar behaviors of some non-Newtonian fluids.

We first describe the rod-climbing effect (see Figure2 2 or R.G. Owens and T.N. Phillips 103 Fig 1.9]). A rod is introduced in the fluid and is rotated: for a Newtonian fluid, inertia causes the fluid to dip near the rod and rise at the walls. For some non-Newtonian fluids, the fluid may actually climb the rod (this is called the Weissenberg effect). This phenomenon is related to non zero normal stress differences (see A.S. Lodge [90]).

Another experiment is the open syphon effect (see Figure 2]or R.G. Owens and T.N. Phillips [103, Fig. 1.11]). A beaker is tilted so that a small thread of the fluid starts to flow over the edge, and then is put straight again. For some viscoelastic fluids, the liquid keeps on flowing out.

Another, simpler experiment, which we will be able to reproduce with a micro-macro model and a simple numerical computation (see Section 5) is the start-up of shear flow. A fluid initially at rest and confined between two plates is sheared (one plate is moving, and the other one is fixed) (see Figures 1 and 3). For Newtonian fluids, the velocity profile progressively reaches monotonically the stationary state. For some polymeric fluids, the velocity goes beyond its stationary value: this is the overshoot phenomenon. 

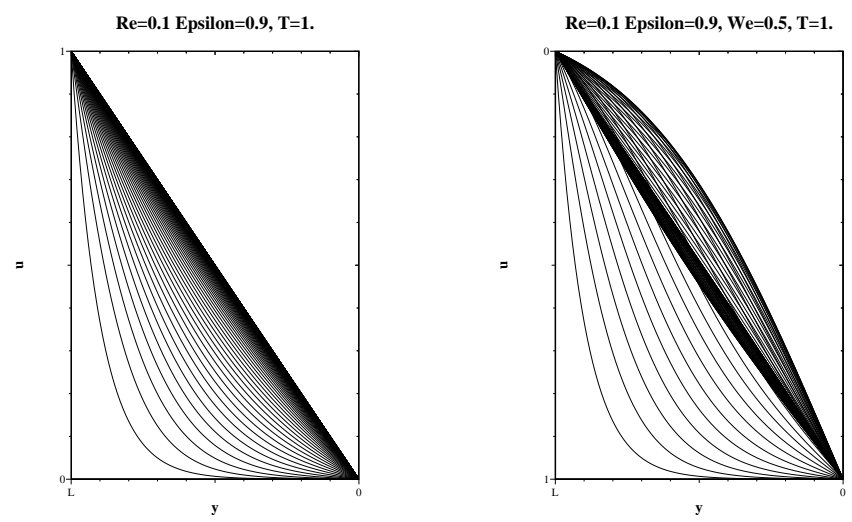

Figure 3: Velocity profile as a function of time for a start-up of shear flow. The velocity profile ( $u$ as a function of $y$, see Figure 1 ) is represented at various times in the time interval $[0,1]$. For polymeric fluids (on the right, case of the Hookean dumbbell micro-macro model) an overshoot of the velocity is observed, while this is not the case for Newtonian fluid (on the left).

\subsubsection{Modelling of non-Newtonian fluids}

When the fluid, although viscous, incompressible and homogeneous, does not obey the simplifying assumptions leading to (2.5), the following system of equations is to be used, in lieu of (2.9)-(2.10):

$$
\left\{\begin{aligned}
\rho\left(\frac{\partial \boldsymbol{u}}{\partial t}+(\boldsymbol{u} \cdot \nabla) \boldsymbol{u}\right)-\eta \Delta \boldsymbol{u}+\nabla p-\operatorname{div} \boldsymbol{\tau}_{p} & =\rho \boldsymbol{f} \\
\operatorname{div} \boldsymbol{u} & =0
\end{aligned}\right.
$$

where the stress $\tau$ has been decomposed along

$$
\boldsymbol{\tau}=\boldsymbol{\tau}_{n}+\boldsymbol{\tau}_{p}
$$

giving birth to the terms $-\eta \Delta \boldsymbol{u}$, and $\operatorname{div} \boldsymbol{\tau}_{p}$, respectively. In (2.14), $\boldsymbol{\tau}_{n}$ denotes the Newtonian contribution (expressed as in (2.5) and $\tau_{p}$ denotes the part of the stress (called non-Newtonian or extra stress) which cannot be modelled as in (2.4). Our notation $\tau_{p}$ refers to the fact we will mainly consider in the sequel fluids for which the non-Newtonian character owes to the presence of polymeric chains flowing in a solvent.

For non-Newtonian fluids, many purely macroscopic models exist. All are based upon considerations of continuum mechanics. The bottom line is to write an equation, in the 
vein of (2.4), ruling the evolution of the non-Newtonian contribution $\tau_{p}$ to the stress tensor, and/or a relation between the latter and other quantities characterizing the fluid dynamics, such as the deformation tensor $\boldsymbol{d}$ or $\nabla \boldsymbol{u}$ itself. Such an equation may read

$$
\frac{D \boldsymbol{\tau}_{p}}{D t}=F\left(\boldsymbol{\tau}_{p}, \nabla \boldsymbol{u}\right)
$$

where $\frac{D \bullet}{D t}$ denotes an appropriate extension (for tensorial quantities, see next section) of the usual convected derivative for vectors

$$
\frac{\partial \bullet}{\partial t}+(\boldsymbol{u} \cdot \nabla) \bullet .
$$

A model such as (2.15) is called a differential model for the non-Newtonian fluid. One famous example is the Oldroyd B model. It will be made precise in the next section.

An alternative option is to resort to an integral model:

$$
\boldsymbol{\tau}_{p}(t, \boldsymbol{x})=\int_{-\infty}^{t} m\left(t-t^{\prime}\right) \boldsymbol{S}_{t^{\prime}} d t^{\prime}
$$

where $\boldsymbol{S}_{t^{\prime}}$ denotes a quantity depending on $\nabla \boldsymbol{u}$, and where the integral is considered along a fluid trajectory (or pathline) ending at point $\boldsymbol{x}$. We shall also return to such models in the next section.

The major observation on both forms $(2.15)$ and $(2.16)$ is that, in contrast to the Newtonian case (2.5), $\boldsymbol{\tau}_{p}(t, \boldsymbol{x})$ does not only depend on the deformation at point $\boldsymbol{x}$ and at time $t$ (as it would be the case in (2.5)), but also depends on the history of the deformation for all times $t^{\prime} \leq t$, along the fluid trajectory leading to $\boldsymbol{x}$. It is particularly explicit on the form (2.16), but may also be seen on 2.15.

The complete system of equations modelling the fluid reads

$$
\left\{\begin{aligned}
\rho\left(\frac{\partial \boldsymbol{u}}{\partial t}+(\boldsymbol{u} \cdot \nabla) \boldsymbol{u}\right)-\eta \Delta \boldsymbol{u}+\nabla p-\operatorname{div} \boldsymbol{\tau}_{p} & =\rho \boldsymbol{f} \\
\frac{\operatorname{div} \boldsymbol{u}}{D \boldsymbol{\tau}_{p}} & =0 \\
D t & =F\left(\boldsymbol{\tau}_{p}, \nabla \boldsymbol{u}\right) .
\end{aligned}\right.
$$

This system is called a three-field system. It involves the velocity $\boldsymbol{u}$, the pressure $p$, and the stress $\tau_{p}$.

Solving this three-field problem is much more difficult and computationally demanding than the 'simple' Newtonian problem (2.9)-(2.10), that is (2.13) where $\tau_{p}=0$ and only two fields, the velocity and the pressure, are to be determined. However, the major scientific difficulty is neither a mathematical one nor a computational one. The major difficulty is to derive an equation of the type (2.15) or (2.16). It requires a deep, qualitative and 
quantitative, understanding of the physical properties of the fluid under consideration. For many non-Newtonian fluids, complex in nature, reaching such an understanding is a challenge. Moreover, even if such an equation is approximately known, evaluating the impact of its possible flaws on the final result of the simulation is not an easy matter. It can only be done a posteriori, comparing the results to actual experimental observations, when the latter exist, and they do not always exist. The difficulty is all the more prominent that the non-Newtonian fluids are very diverse in nature. New materials appear on a daily basis. For traditional fluids considered under unusual circumstances, or for recently (or even not yet) synthesized fluids, reliable evolution equations are not necessarily available.

All this, in its own, motivates the need for alternative strategies, based on an explicit microscopic modelling of the fluid. This will give rise to the so-called micro-macro models, which are the main topic of this article. The lack of information at the macroscopic level is then circumvented by a multiscale strategy consisting in searching for the information at a finer level (where reliable models do exist, based on general conservation equations, posed $e . g$. on the microstructures of the fluids). The latter information is then inserted in the equations of conservation at the macroscopic level. At the end of the day, because the modelling assumptions are avoided as much as possible, a complete description is attained, based on a more reliable, however much more computationally demanding, model. Otherwise stated, a crucial step of the modelling is replaced by a numerical simulation. But before we turn to this, from Section 3 on, let us give some more details on the purely macroscopic models (2.17) for non-Newtonian fluids. They are today the most commonly used models (in particular because they are less demanding computationally). For our explanatory survey, we choose the context of viscoelastic fluids.

\subsection{Some macroscopic models for viscoelastic fluids}

Throughout this section, the stress tensor $\tau$ is decomposed into a Newtonian part and a non-Newtonian part, as in (2.14). The former, $\boldsymbol{\tau}_{n}$, reads $\boldsymbol{\tau}_{n}=\eta \dot{\gamma}$ where $\eta$ is the viscosity, and $\dot{\gamma}$ is given by

$$
\dot{\gamma}=\nabla \boldsymbol{u}+\nabla \boldsymbol{u}^{T}
$$

The latter is denoted by $\tau_{p}$. The stress is the combination of the two, namely:

$$
\tau=\eta \dot{\gamma}+\tau_{p}
$$

\subsubsection{More on differential models}

The basic model for viscoelastic fluids is the Maxwell model. It combines a linear elasticity model and a linear viscosity model. In the former, the stress depends linearly on the deformation. It is the Hooke law. The proportionality constant is the Young modulus E. This part of the stress is to be thought of as a linear spring. On the other hand, the linear viscosity model assumes the stress depends linearly on the rate (or speed) of deformation, the proportionality constant being the viscosity $\eta$. Heuristically, this amounts to considering 


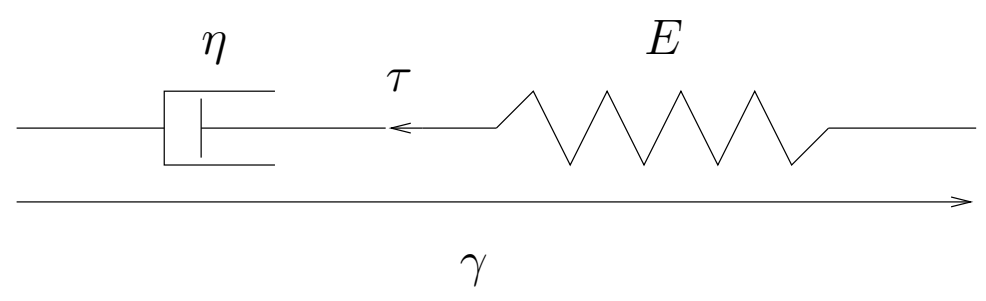

Figure 4: One-dimensional Maxwell model. The analogy with an electric circuit is obvious, $\tau$ and $\gamma$ playing the role of the intensity and the voltage respectively, $\eta$ and $E$ that of the capacity of a capacitor and the conductivity of a resistor respectively.

a piston. The one-dimensional Maxwell model combines the Hookean spring and the piston (see Figure 4). Then, denoting the stress by $\tau$ and the deformation rate by $\dot{\gamma}$, the following ordinary differential equation is obtained:

$$
\dot{\gamma}=\frac{1}{E} \frac{d \tau}{d t}+\frac{\tau}{\eta}
$$

that is,

$$
\lambda \frac{d \tau}{d t}+\tau=\eta \dot{\gamma},
$$

where $\lambda=\frac{\eta}{E}$ denotes a characteristic relaxation time of the system.

Remark 2.1 The mathematically inclined reader should not be surprised by the elementary nature of the above arguments. Modelling is simplifying. Some excellent models of fluid mechanics (and other fields of the engineering and life sciences) are often obtained using such simple derivations. On the other hand, it is intuitively clear that the determination of the parameters of such models is often an issue, which limits their applicability, and that there is room for improvement using more advanced descriptions of matter. This will be the purpose of the multiscale models introduced in the present article.

Passing from the one-dimensional setting to higher dimensions requires to replace the time derivative in (2.21) by a convective derivative of a tensor. Based on invariance arguments, the following model is derived:

$$
\lambda\left(\frac{\partial \boldsymbol{\tau}_{p}}{\partial t}+\boldsymbol{u} \cdot \nabla \boldsymbol{\tau}_{p}-\nabla \boldsymbol{u} \boldsymbol{\tau}_{p}-\boldsymbol{\tau}_{p}(\nabla \boldsymbol{u})^{T}\right)+\boldsymbol{\tau}_{p}=\eta_{p} \dot{\boldsymbol{\gamma}},
$$

where $\lambda$ is a relaxation time, and $\eta_{p}$ is an extra viscosity (due to the polymers in our context). Then the stress tensor $\tau$ is given by (2.19). When $\eta=0$, the model is called the Upper Convected Maxwell model (UCM). When $\eta \neq 0$, it is the Oldroyd-B model, also called the Jeffreys model. 
For future reference, let us rewrite the complete system of equations for the Oldroyd-B model, in a non-dimensional form:

$$
\left\{\begin{array}{l}
\operatorname{Re}\left(\frac{\partial \boldsymbol{u}}{\partial t}+\boldsymbol{u} \cdot \nabla \boldsymbol{u}\right)=(1-\epsilon) \Delta \boldsymbol{u}-\nabla p+\operatorname{div} \boldsymbol{\tau}_{p} \\
\operatorname{div} \boldsymbol{u}=0 \\
\frac{\partial \boldsymbol{\tau}_{p}}{\partial t}+\boldsymbol{u} \cdot \nabla \boldsymbol{\tau}_{p}-(\nabla \boldsymbol{u}) \boldsymbol{\tau}_{p}-\boldsymbol{\tau}_{p}(\nabla \boldsymbol{u})^{T}=-\frac{1}{\mathrm{We}} \boldsymbol{\tau}_{p}+\frac{\epsilon}{\mathrm{We}}\left(\nabla \boldsymbol{u}+(\nabla \boldsymbol{u})^{T}\right) .
\end{array}\right.
$$

The Reynolds number Re $>0$, the Weissenberg number We $>0$ and $\epsilon \in(0,1)$ are the non-dimensional numbers of the system (see Equations 4.45) below for precise definitions). The Weissenberg number (which is the ratio of the characteristic relaxation time of the microstructures in the fluid to the characteristic time of the fluid) plays a crucial role in the stability of numerical simulations (see Section 4.4).

Remark 2.2 In (2.22) and throughout this article, we denote by $(\nabla \boldsymbol{u})_{i, j}=\frac{\partial u_{i}}{\partial x_{j}}$. Other, and in fact many authors in the literature of non-Newtonian fluid mechanics (see e.g. R.B. Bird, C.F. Curtiss, R.C. Armstrong and O. Hassager [11, 12], R.G. Owens and T.N. Phillips [103], or H.C. Öttinger [101])), adopt the alternative convention: $(\nabla \boldsymbol{u})_{i, j}=\frac{\partial u_{j}}{\partial x_{i}}$. Our equations have to be modified correspondingly.

Remark 2.3 The convective derivative in [2.22) is called the upper-convected derivative. Other derivatives may be considered, such as the lower-convected derivative, or the corotational derivative (the latter being particularly interesting for mathematical purposes, see Section (6). All these derivatives obey the appropriate invariance laws of mechanics, but we have chosen the upper-convected derivative because it spontaneously arises when using the kinetic models (see Section [3). It is also the most commonly used derivative in macroscopic models, such as the Phan-Thien Tanner model, the Giesekus model or the FENE-P model. We shall return to such models later on. A discussion of the physical relevance of convective derivatives appears in D. Bernardin [10, chapter 3]. See also R.B. Bird, R.C. Armstrong and O. Hassager [11, Chapter 9].

The Oldroyd B model has several flaws, as regards its ability to reproduce experimentally observed behaviors.

Refined macroscopic models for viscoelastic fluids have thus been derived, allowing for a better agreement between simulation and experience. In full generality, such models read:

$$
\lambda\left(\frac{\partial \boldsymbol{\tau}_{p}}{\partial t}+\boldsymbol{u} \cdot \nabla \boldsymbol{\tau}_{p}-\nabla \boldsymbol{u} \boldsymbol{\tau}_{p}-\boldsymbol{\tau}_{p}(\nabla \boldsymbol{u})^{T}\right)+\boldsymbol{T}\left(\boldsymbol{\tau}_{p}, \dot{\gamma}\right)=\eta_{p} \dot{\gamma}
$$

where $\boldsymbol{T}\left(\boldsymbol{\tau}_{p}, \dot{\gamma}\right)$ typically depends nonlinearly on $\boldsymbol{\tau}_{p}$. The most commonly used models are the following three. The Giesekus model(see H. Giesekus [51]) involves a quadratic term:

$$
\lambda\left(\frac{\partial \boldsymbol{\tau}_{p}}{\partial t}+\boldsymbol{u} \cdot \nabla \boldsymbol{\tau}_{p}-\nabla \boldsymbol{u} \boldsymbol{\tau}_{p}-\boldsymbol{\tau}_{p}(\nabla \boldsymbol{u})^{T}\right)+\boldsymbol{\tau}_{p}+\alpha \frac{\lambda}{\eta_{p}} \boldsymbol{\tau}_{p} \boldsymbol{\tau}_{p}=\eta_{p} \dot{\boldsymbol{\gamma}}
$$


where $\alpha$ is a fixed scalar.

The Phan-Thien Tanner model (PTT) is derived from a lattice model (see N. Phan-Thien and R.I. Tanner [105]). It writes:

$$
\lambda\left(\frac{\partial \boldsymbol{\tau}_{p}}{\partial t}+\boldsymbol{u} \cdot \nabla \boldsymbol{\tau}_{p}-\nabla \boldsymbol{u} \boldsymbol{\tau}_{p}-\boldsymbol{\tau}_{p}(\nabla \boldsymbol{u})^{T}\right)+Z\left(\operatorname{tr}\left(\boldsymbol{\tau}_{p}\right)\right) \boldsymbol{\tau}_{p}+\frac{\xi}{2} \lambda\left(\dot{\gamma} \boldsymbol{\tau}_{p}+\boldsymbol{\tau}_{p} \dot{\gamma}\right)=\eta_{p} \dot{\boldsymbol{\gamma}},
$$

with two choices for the function $Z$ :

$$
Z\left(\operatorname{tr}\left(\boldsymbol{\tau}_{p}\right)\right)=\mid \begin{aligned}
& 1+\phi \lambda \frac{\operatorname{tr}\left(\boldsymbol{\tau}_{p}\right)}{\eta_{p}} \\
& \exp \left(\phi \lambda \frac{\operatorname{tr}\left(\boldsymbol{\tau}_{p}\right)}{\eta_{p}}\right)
\end{aligned},
$$

where $\xi$ and $\phi$ are fixed scalars.

The FENE-P model, which we will return to in Section 4.2 is derived from a kinetic model (see A. Peterlin [104 and R.B. Bird, P.J. Dotson and N.L. Johnson [13] and Section 4]. It reads:

$$
\left\{\begin{array}{l}
\lambda\left(\frac{\partial \boldsymbol{\tau}_{p}}{\partial t}+\boldsymbol{u} \cdot \nabla \boldsymbol{\tau}_{p}-\nabla \boldsymbol{u} \boldsymbol{\tau}_{p}-\boldsymbol{\tau}_{p}(\nabla \boldsymbol{u})^{T}\right)+Z\left(\operatorname{tr}\left(\boldsymbol{\tau}_{p}\right)\right) \boldsymbol{\tau}_{p} \\
\quad-\lambda\left(\boldsymbol{\tau}_{p}+\frac{\eta_{p}}{\lambda} \operatorname{Id}\right)\left(\left(\frac{\partial}{\partial t}+\boldsymbol{u} \cdot \nabla\right) \ln \left(Z\left(\operatorname{tr}\left(\boldsymbol{\tau}_{p}\right)\right)\right)\right)=\eta_{p} \dot{\boldsymbol{\gamma}}
\end{array}\right.
$$

with

$$
Z\left(\operatorname{tr}\left(\boldsymbol{\tau}_{p}\right)\right)=1+\frac{d}{b}\left(1+\lambda \frac{\operatorname{tr}\left(\boldsymbol{\tau}_{p}\right)}{d \eta_{p}}\right)
$$

where $d$ is the dimension of the ambient space and $b$ is a scalar that is thought of as related to the maximal extensibility of the polymer chains embedded in the fluids (see the FENE force below, Equation (4.39).

All these nonlinear models yield much better results than the Oldroyd B model, and satisfactorily agree with experiments on simple flows. They can be further generalized, considering several relaxation times $\lambda_{i}$ and several viscosities $\eta_{p, i}$, but we will not proceed further in this direction in this introductory survey.

\subsubsection{More on integral models}

Let us return to the one-dimensional Maxwell model (2.21). Its solution may be explicitly written in terms of the integral:

$$
\tau(t)=\tau\left(t_{0}\right) \exp \left(-\frac{t-t_{0}}{\lambda}\right)+\int_{t_{0}}^{t} \frac{\eta}{\lambda} \exp \left(-\frac{t-s}{\lambda}\right) \dot{\gamma}(s) d s .
$$

Letting $t_{0}$ go to $-\infty$, and assuming $\tau$ bounded when $\dot{\gamma}$ is bounded, we obtain:

$$
\tau(t)=\int_{-\infty}^{t} \frac{\eta}{\lambda} \exp \left(-\frac{t-s}{\lambda}\right) \dot{\gamma}(s) d s .
$$


Denoting by:

$$
\left\{\begin{array}{l}
\frac{d}{d t} \gamma\left(t_{0}, t\right)=\dot{\gamma}(t) \\
\gamma\left(t_{0}, t_{0}\right)=0
\end{array}\right.
$$

and integrating by parts, we obtain a form equivalent to (2.31) :

$$
\tau(t)=\int_{-\infty}^{t} \frac{\eta}{\lambda^{2}} \exp \left(-\frac{t-s}{\lambda}\right) \gamma(t, s) d s .
$$

This form explicitly shows that, as announced earlier, the constraint at time $t$ depends on the history of the deformation. The function $\frac{\eta}{\lambda^{2}} \exp \left(-\frac{t-s}{\lambda}\right)$ is often called a memory function.

The one-dimensional computation performed above can be generalized to higher dimensions and yields:

$$
\boldsymbol{\tau}_{p}(t, \boldsymbol{x})=-\int_{-\infty}^{t} M(t-s) f\left(\boldsymbol{C}_{t}^{-1}(s, \boldsymbol{x})\right)\left(\operatorname{Id}-\boldsymbol{C}_{t}^{-1}(s, \boldsymbol{x})\right) d s .
$$

where $M$ is a memory function, $f$ is a given real valued function, and $\boldsymbol{C}_{t}^{-1}(s, \boldsymbol{x})$ denotes the so-called Finger tensor (at time $t$ ). The latter is the inverse tensor of the Cauchy deformation tensor:

$$
\boldsymbol{C}_{t}(s, \boldsymbol{x})=\boldsymbol{F}_{t}(s, \boldsymbol{x})^{T} \boldsymbol{F}_{t}(s, \boldsymbol{x})
$$

where

$$
\boldsymbol{F}_{t}(s, \boldsymbol{x})=\nabla\left(\boldsymbol{\chi}_{t}(s)\right)(\boldsymbol{x})
$$

is the deformation tensor and $\chi_{t}(s)$ is the flow chart (mapping positions at time $t$ to positions at time $s$ ).

It is easily seen that the upper-convected derivative of the Finger tensor vanishes. When $M(t-s)=\frac{\eta_{p}}{\lambda^{2}} \exp \left(-\frac{t-s}{\lambda}\right)$ and $f=1$, this shows that $\tau_{p}$ defined by (2.34) satisfies (2.22). The parameter $\lambda$ models the time needed by the system to "forget" the history of the deformation.

Remark 2.4 As in the case of differential models, there exist many generalizations and variants for the integral models introduced above. Alternative convective derivatives may be considered, several characteristic times $\lambda_{i}$ and viscosities $\eta_{p, i}$ can be employed. See R.B. Bird, R.C. Armstrong and O. Hassager [11] or D. Bernardin [10] for such extensions. 


\section{Multiscale modelling of polymeric fluids}

There exists an incredibly large variety of non-Newtonian fluids. We have briefly overviewed in the previous section the modelling of viscoelastic fluids. This is one category of nonNewtonian fluids. One important class of non-Newtonian fluids is the family of fluids with microstructures. For such fluids, the non-Newtonian character owes to the presence of microstructures, often more at a mesoscopic scale than at a truly microscopic one. Snow, blood, liquid concrete, etc, are examples of fluids with microstructures. Polymeric fluids form the category we will focus on in the sequel. Analogous scientific endeavors can be followed for other fluids with microstructures. The bottom line for the modelling remains: write an equation at the microscopic level that describes the evolution of the microstructures, then deduce the non-Newtonian contribution $\boldsymbol{\tau}_{p}$ to the stress. Thus a better quantitative understanding. Section 7 will give some insight on other types of fluids with microstructures.

The present section is only a brief introduction to the subject. To read more on the multiscale modelling of complex fluids, we refer to the monographs: R. Bird, Ch. Curtiss, C. Armstrong and O. Hassager 11, 12, HC. Ottinger 101, R. Owens and T. Phillips 103]. Other relevant references from the physics perspective are F. Devreux [34]. M. Doi [35], M. Doi and S.F. Edwards [36], M.P. Allen and D.J. Tildesley [1, D. Frenkel and B. Smit [47].

Before we get to the heart of the matter, let us briefly introduce the reader to the specificities of polymeric fluids.

A polymer is, by definition, a molecular system formed by the repetition of a large number of molecular subsystems, the monomers, all bound together by intramolecular forces. If the subsystems are not all of the same chemical type, one speaks of copolymers. Polymeric materials are ubiquitous: they may be of natural origin, such as natural rubber, wood, leather, or artificially synthesized, such as vulcanized rubber or plastic. They can be classified according to their polymerization degree, that is the number $N$ of monomers present in the chain: $N=1$ to 4 for gases, $N=5$ for oils, $N=25$ for brittle materials such as a candle, $N>2000$ for ductile materials such as plastic films. As $N$ grows, the fusion temperature grows and the polymeric properties become prominent: they are already significant for $N=100$, and obviously dominant for $N=1000$. The specific mechanical properties of the material stem from the long chains present inside. The length of the chain for instance prevents the material from organizing itself regularly when solidification occurs, thus the flexibility of the material (such as a tire). Likewise, the long chains give additional viscosity to liquid polymers, such as oils. Solvents may enjoy good, or bad, solvating properties for the polymers, depending whether the chains expand or retract in the solvent. For example, paints are solvated differently in water and oils.

As regards the concentration of polymers within the solvent, three cases may schematically arise. When the concentration is low, one speaks of infinitely dilute polymeric fluids. There, the chains basically ignore each other, interacting with one another only through the solvent. This is the case we will mainly consider in the sequel. The other extreme case is the case of dense polymeric fluids, also called polymer melts. In-between, one finds polymeric fluids with intermediate concentrations. Of the above three categories, polymer 
melts form indeed the most interesting one, technically and industrially. Their modelling has made great progress in the 1960s with the contributions by De Gennes, and his theory of reptation. Basically, it is considered that, owing to the density of polymeric chains present, each single chain moves in the presence of others like a snake in a dense bush, or a spaghetti in a plate of spaghettis.

Reptation models amount, mathematically, to equations for the evolution of microstructures similar in spirit to those that will be manipulated below. There are however significant differences. Macroscopic versions also exist. In any case, models for polymer melts are much less understood mathematically than models for infinitely dilute polymers. For this reason, we will not proceed further in this direction in the present mathematically biased text.

Remark 3.1 Let us give some details about the reptation model for polymer melts (see for example [101, Section 6]). In such a model, the microscopic variables are $\left(\boldsymbol{U}_{t}, S_{t}\right)$ (say at a fixed position in space $\boldsymbol{x}$ ), where $\boldsymbol{U}_{t}$ is a three dimensional unit vector representing the direction of the reptating polymer chain at the curvilinear abscissa $S_{t}\left(S_{t}\right.$ is a stochastic process with value in $(0,1))$. The Fokker-Planck equation ruling the evolution of $\left(\boldsymbol{U}_{t}, S_{t}\right)$ is:

$$
\begin{aligned}
& \frac{\partial \psi(t, \boldsymbol{x}, \boldsymbol{U}, S)}{\partial t}+\boldsymbol{u}(t, \boldsymbol{x}) \cdot \nabla_{\boldsymbol{x}} \psi(t, \boldsymbol{x}, \boldsymbol{U}, S) \\
& =-\operatorname{div}_{\boldsymbol{U}}\left(\left(\nabla_{\boldsymbol{x}} \boldsymbol{u}(t, \boldsymbol{x}) \boldsymbol{U}-\nabla_{\boldsymbol{x}} \boldsymbol{u}(t, \boldsymbol{x}): \boldsymbol{U} \otimes \boldsymbol{U} \boldsymbol{U}\right) \psi(t, \boldsymbol{x}, \boldsymbol{U}, S)\right) \\
& \quad+\frac{1}{\lambda} \frac{\partial^{2} \psi(t, \boldsymbol{x}, \boldsymbol{U}, S)}{\partial S^{2}} .
\end{aligned}
$$

The boundary conditions for $S=0$ and $S=1$ supplementing the Fokker-Planck equation are

$$
\psi(t, \boldsymbol{x}, \boldsymbol{U}, 0)=\psi(t, \boldsymbol{x}, \boldsymbol{U}, 1)=\frac{1}{4 \pi} \delta_{|\boldsymbol{U}|=1},
$$

where $\delta_{|\boldsymbol{U}|=1}$ is the Lebesgue (surface) measure on the sphere. In terms of the stochastic process $\left(\boldsymbol{U}_{t}, S_{t}\right)$, this equation is formally equivalent to a deterministic evolution of the process $\boldsymbol{U}_{t}$ (the unit vector is rotated following the flow field) and a stochastic evolution of the index $S_{t}$ as $d S_{t}+\boldsymbol{u} \cdot \nabla_{\boldsymbol{x}} S_{t} d t=\sqrt{2 / \lambda} d W_{t}$. The only coupling between $\boldsymbol{U}_{t}$ and $S_{t}$ arises when $S_{t}$ reaches 0 or 1 , in which case $\boldsymbol{U}_{t}$ is reinitialized randomly uniformly on the sphere. The contribution of the polymers to the stress tensor can then be computed using a Kramers formula (similar to (3.12)), and this closes the micro-macro model. An interesting open mathematical question is to define rigorously the dynamics of the process $\left(\boldsymbol{U}_{t}, S_{t}\right)$.

Remark 3.2 Also for concentrated polymers, a regime different from reptation can also be considered. When sufficiently numerous bridges are (chemically) created between the entangled chains (this is exactly the purpose of the vulcanization process involved in the production of tires), the polymeric material turns into a lattice, often called a reticulated polymer. Its properties are intermediate between those of a fluid and those of a solid material (owing to the -slight- rigidity provided by the lattice). A multiscale modeling can be envisioned for such materials, but again this is not the purpose of this article. We refer for example to H. Gao and P. Klein [48], or S. Reese [107, 108]. 


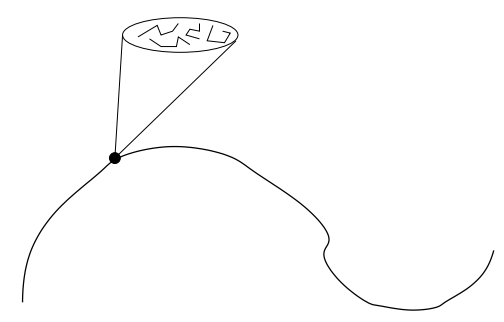

Figure 5: A collection of polymeric chains lies, microscopically, at each macroscopic point of the trajectory of a fluid particle.

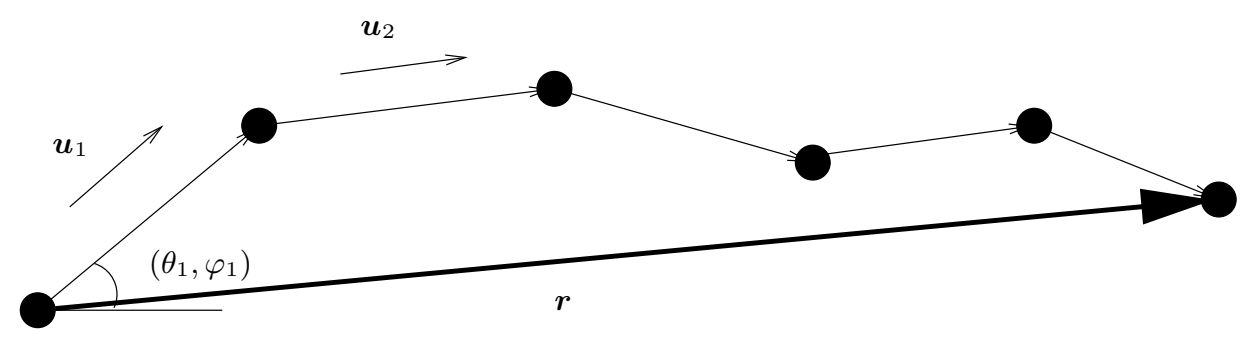

Figure 6: A polymeric chain: $\boldsymbol{u}_{j}$ denote the unit vectors between the "atoms", each of them corresponds to a pair of angles $\left(\theta_{i}, \varphi_{i}\right)$ and has length $a$. The end-to-end vector is $\boldsymbol{r}$.

In the sequel of this article (with the notable exception of Section!7), we consider infinitely dilute polymeric fluids.

In order to understand the contribution to the stress provided by this assembly of long polymeric chains, we zoom out on such a chain. We now want to write an evolution equation on this object. First we have to model the chain, then see the forces it experiences, and finally write an appropriate evolution equation.

As regards the modelling of a polymeric chain, the point to understand is that it is out of the question to explicitly model all the atoms of the chain. There are thousands of them. The interactions between atoms are incredibly expensive to model. Without even thinking to a model from quantum chemistry, the 'simple' consideration of a classical force-field for the molecular dynamics of an entire polymeric chain is too a computationally demanding task. It can be performed for some sufficiently short chains, considered alone, and not interacting with their environment. But the simulation in situ, over time frame relevant for the fluid mechanics simulation, of millions of long chains, is out of reach. Even if it was possible, there is no reason to believe that the actual motion of each single atom, and the precise description of the dynamics of each chain significantly impacts the overall rheology of the fluid. 
So the two keywords here are statistical mechanics and coarse-graining (somehow, these terms are synonymous). The bottom line is to consider one single, hopefully representative chain, sitting at a macropoint of the fluid, then derive some sufficiently simple description of this chain, which carries enough physics to adequately model the impact of the chains onto the fluid, and conversely. This within the limit of our simulation capabilities.

Let us first obtain a coarse-grained model for the chain.

\subsection{Statistical mechanics of the free chain}

\subsubsection{Generalities}

As said above, it is not reasonable, and it is not the point, to simulate the actual dynamics of all atoms composing all the chains.

We first choose a representative chain. For simplicity, we assume the chain is a linear arrangement of $N$ beads (as opposed to the case of branched polymers, where the chain has several branches). Each of these beads models a group of atoms, say 10 to 20 monomer units. They are milestones along the chain. They are assumed to be connected by massless bars with length $a$. This is the so-called Kramers chain model (see Figure 6). The configuration of the chain, at time $t$ and each macroscopic point $\boldsymbol{x}$, is described by a probability density $\psi$ (momentarily implicitly indexed by $t$ and $\boldsymbol{x}$ ), defined over the space

$$
\left(\theta_{1}, \varphi_{1}, \ldots, \theta_{N-1}, \varphi_{N-1}\right)
$$

of Euler angles of the unit vectors $\boldsymbol{u}_{i}$ along this representative chain. Thus

$$
\psi\left(\theta_{1}, \varphi_{1}, \ldots, \theta_{N-1}, \varphi_{N-1}\right) d \theta_{1} d \varphi_{1} \ldots d \theta_{N-1} d \varphi_{N-1}
$$

is the probability that the chain has angles between $\left(\theta_{1}, \varphi_{1}\right)$ and $\left(\theta_{1}+d \theta_{1}, \varphi_{1}+d \varphi_{1}\right)$ between the first two beads labeled 1 and 2, etc...

Some coarse graining has already been performed by considering these beads instead of the actual atoms, but we will now proceed one step further. We are going to only keep a very limited number of these beads, say $N_{b}$, and eliminate (using a limiting procedure) all the $N-N_{b}$ beads in-between. The typical number of beads kept is well below 100. The simplest possible case, that of $N_{b}=2$ beads, is the dumbbell case and we will in fact mainly concentrate on it in the sequel.

In order to reduce the description of the chain to the simple knowledge of $N_{b}=2$ beads, we are going to consider the vector $r$ linking the first bead to the last one. This vector is called the end-to-end vector (see Figure 6) and writes as the sum

$$
\boldsymbol{r}=\sum_{i=1}^{N-1} a \boldsymbol{u}_{i},
$$

where $\boldsymbol{u}_{i}$ is the unit vector describing the $i$-th link. Between the extreme two beads lies indeed a supposedly large number $N-2$ of beads. The chain is free to rotate around 
each of these beads: think typically to the arm of a mechanical robot. We first describe all the possible configurations of all the $N$ beads. In a second step, we will pass to the limit $N \longrightarrow+\infty$ in order to obtained a reduced model for the two extreme beads, thereby obtaining a statistics on the end-to-end vector. The motivation for this limit process is of course that, given the two extreme beads, the $N-2$ other beads are in extremely large number.

At equilibrium (namely for zero velocity field for the surrounding solvent and at a fixed temperature), the probability density for the Euler angles $\left(\theta_{i}, \varphi_{i}\right)$ of the $i$-th link writes

$$
\psi_{i}\left(\theta_{i}, \varphi_{i}\right)=\frac{1}{4 \pi} \sin \theta_{i}
$$

simply by equiprobability of the orientation of this link. As the chain freely rotates around each bead, the orientations of links are independent from one link to another one, and thus the overall probability density for the sequence of angles $\left(\theta_{1}, \varphi_{1}, \ldots, \theta_{N-1}, \varphi_{N-1}\right)$ is simply the product

$$
\psi\left(\theta_{1}, \varphi_{1}, \ldots, \theta_{N-1}, \varphi_{N-1}\right)=\left(\frac{1}{4 \pi}\right)^{N-1} \prod_{i=1}^{N-1} \sin \theta_{i} .
$$

Any statistical quantity (observable) $B$ depending on the state of the chain thus has average value

$$
\langle B\rangle=\int B\left(\theta^{N-1}, \varphi^{N-1}\right) \psi\left(\theta^{N-1}, \varphi^{N-1}\right) d \theta^{N-1} d \varphi^{N-1}
$$

where $\theta^{N-1}=\left(\theta_{1}, \ldots, \theta_{N-1}\right)$ and $\varphi^{N-1}=\left(\varphi_{1}, \ldots, \varphi_{N-1}\right)$.

For instance, it is a simple calculation that

$$
\left\langle|\boldsymbol{r}|^{2}\right\rangle=(N-1) a^{2}
$$

where $a$ denotes the length between two beads.

It follows that the probability density for the end-to-end vector $\boldsymbol{r}$ reads:

$$
P(\boldsymbol{r})=\int \delta\left(\boldsymbol{r}-\sum_{i=1}^{N-1} a \boldsymbol{u}_{i}\right) \psi\left(\theta^{N-1}, \varphi^{N-1}\right) d \theta^{N-1} d \varphi^{N-1},
$$

where $\delta$ is formally a Dirac mass and $\boldsymbol{u}_{i}$ the unit vector of Euler angles $\left(\theta_{i}, \varphi_{i}\right)$. Using (3.3), a simple but somewhat tedious calculation shows that an adequate approximation formula for $P$, in the limit of a large number $N-2$ of beads eliminated, is

$$
P(\boldsymbol{r}) \stackrel{N \operatorname{large}}{\approx}\left(\frac{3}{2 \pi(N-1) a^{2}}\right)^{3 / 2} \exp \left(-\frac{3|\boldsymbol{r}|^{2}}{2(N-1) a^{2}}\right) .
$$

The right-hand side of (3.7) is now chosen to be the probability law of $r$, which is consequently a Gaussian variable. From then on, only the end-to-end vector, and its probability, are kept as the statistical description of the entire chain. 


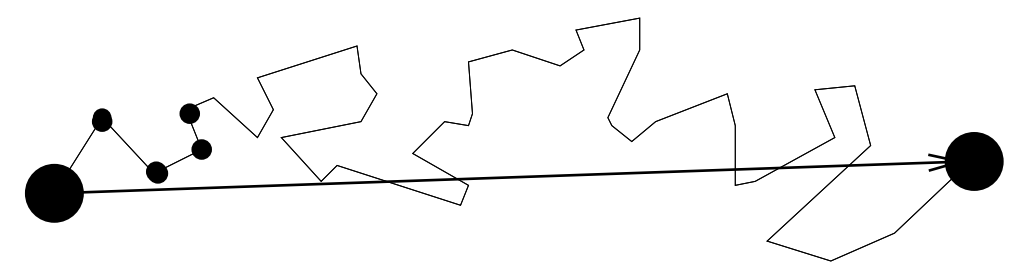

Figure 7: A polymeric chain consisting of, say, thirty beads and its phenomenological representation as a dumbbell.

Remark 3.3 For some dedicated applications, chains with $N_{b}=10$ or $N_{b}=20$ beads are simulated. This is typically the case when one wants to model multiple relaxation time scales in the polymer chain or understand boundary effects. Consider a pipeline where the polymeric fluid flows. Some macroscopic model may provide good results for the inner flow, but they need to be supplied with adequate boundary conditions on the walls of the pipeline. Dumbbell models could be envisioned for this purpose, but since the complexity of the chain is a key issue for rheological properties near the boundaries, more sophisticated models with larger $N_{b}$ have sometimes to be employed. Apart from such specific situations, it is considered that the dumbbell model already gives excellent answers. But this also depends upon the force fields that will be used. The purpose of the next section is exactly to introduce such a force. Others will be mentioned in Section 4.

\subsubsection{The Hookean model}

We now have our configuration space, namely that of a single end-to-end vector $\boldsymbol{r}$ equipped with a Gaussian probability at equilibrium. Let us next define the forces this end-to-end vector experiences.

We need to equip the vector $r$ with some rigidity. Such a rigidity does not express a mechanical rigidity due to forces, of interatomic nature, holding between beads. It will rather model an entropic rigidity, related to the variations of the configurations of the actual entire chain when the end-to-end vector itself varies.

To understand this, let us only mention two extreme situations. If the end-to-end vector has length exactly $|\boldsymbol{r}|=(N-1) a$, there is one and only one configuration of the entire chain that corresponds to such an end-to-end vector, namely the chain fully extended as a straight line. In contrast, when the end-to-end vector has, say, length $|\boldsymbol{r}|=(N-1) a / 2$, there is an enormous number of configurations, corresponding to various shapes of a chain of total length $(N-1) a$ that give rise to such an end-to-end vector. Entropy will thus favor short end-to-end vectors, rather than long ones. It remains now to quantitatively understand this.

We know from Statistical Mechanics arguments that for a system with probability law $P(\boldsymbol{r})$ (obtained from (3.7) ), the free energy is given by

$$
A(\boldsymbol{r})=A^{0}-k T \ln P(\boldsymbol{r})
$$


where $T$ denotes temperature, $A^{0}$ is a constant and $k$ the Boltzmann constant. When the end-to-end vector is modified by $d \boldsymbol{r}$, the resulting free energy modification reads

$$
\begin{aligned}
d A & =-k T d \ln P(\boldsymbol{r}), \\
& =\frac{3 k T}{(N-1) a^{2}} \boldsymbol{r} \cdot d \boldsymbol{r} .
\end{aligned}
$$

On the other hand, when temperature is kept constant, the free energy change is related to the tension $\boldsymbol{F}$ of the chain by

$$
d A=\boldsymbol{F}(\boldsymbol{r}) \cdot d \boldsymbol{r} .
$$

Comparing (3.8) and (3.9), we obtain the tension

$$
\boldsymbol{F}(\boldsymbol{r})=\frac{3 k T}{(N-1) a^{2}} \boldsymbol{r} .
$$

In other words, the entropic force $\boldsymbol{F}$ expressed in terms of the end-to-end vector $\boldsymbol{r}$ is defined as the gradient of $\ln P$ with respect to $r$ where $P$ is the probability density of the end-to-end vector at equilibrium (zero velocity field for the surrounding solvent, and fixed temperature). This definition of the entropic force is consistent with the fact that $P$ is indeed a stationary solution for the dynamics that will be defined on the probability density $\psi$ of the end-to-end vector (see Equation (3.11) below) when the velocity field in the solvent is not zero (out-of-equilibrium situation).

The end-to-end vector therefore acts as a linear spring, with stiffness

$$
H=\frac{3 k T}{(N-1) a^{2}} .
$$

The model obtained is called the Hookean dumbbell model.

The above derivation is the simplest possible one, based on oversimplifying assumptions. Several improvements of the Hookean force (3.10) are indeed possible. We prefer to postpone the presentation of such improvements until Section 4 Let us momentarily assume we have a force $\boldsymbol{F}(\boldsymbol{r})$ at hand, the prototypical example being the Hookean force (3.10), and proceed further. On purpose, we do not make precise the expression of $\boldsymbol{F}(\boldsymbol{r})$ in the sequel.

\subsection{The multiscale model}

Let us now denote $\psi(t, \boldsymbol{x}, \boldsymbol{r})$ the probability density for the end-to-end vectors of the polymer chains at macropoint $\boldsymbol{x}$ and time $t$.

The variation of $\psi$ in time, calculated along a fluid trajectory, that is $\frac{\partial \psi}{\partial t}+\boldsymbol{u} \cdot \nabla_{\boldsymbol{x}} \psi$, follows from three different phenomena:

1. a hydrodynamic force: the dumbbell is elongated or shortened because of the interaction with the fluid ; Its two ends do not necessarily share the same macroscopic velocity, the slight difference in velocities (basically $\nabla \boldsymbol{u}(t, \boldsymbol{x}) \boldsymbol{r}$ ) results in a force elongating the dumbbell $\zeta \nabla \boldsymbol{u}(t, \boldsymbol{x}) \boldsymbol{r}$ where $\zeta$ denotes a friction coefficient; 
2. an entropic force issued from the coarse-graining procedure and which is reminiscent of the actual, much more complex, geometry of the entire polymeric chain;

3. a Brownian force, modelling the permanent collisions of the polymeric chain by solvent molecules, which (randomly) modifies its evolution.

We have gone into many details about the second phenomenon (the entropic force) in the previous section. We will momentarily admit the modelling proposed above for the first and third phenomena (the hydrodynamic force and the Brownian force, respectively) and proceed further. In Section 4 we will return to this in more details, explaining the intimate nature of these forces and motivating their actual mathematical form by rigorous arguments.

The overall conservation of momentum equation reads as the following evolution equation on $\psi$ :

$$
\begin{aligned}
& \frac{\partial \psi(t, \boldsymbol{x}, \boldsymbol{r})}{\partial t}+\boldsymbol{u}(t, \boldsymbol{x}) \cdot \nabla_{\boldsymbol{x}} \psi(t, \boldsymbol{x}, \boldsymbol{r}) \\
& \quad=-\operatorname{div}_{\boldsymbol{r}}\left(\left(\nabla_{\boldsymbol{x}} \boldsymbol{u}(t, \boldsymbol{x}) \boldsymbol{r}-\frac{2}{\zeta} \boldsymbol{F}(\boldsymbol{r})\right) \psi(t, \boldsymbol{x}, \boldsymbol{r})\right)+\frac{2 k T}{\zeta} \Delta_{\boldsymbol{r}} \psi(t, \boldsymbol{x}, \boldsymbol{r}) .
\end{aligned}
$$

Equation (3.11) is called a Fokker-Planck equation (or also a forward Kolmogorov equation). The three terms of the right-hand side of (3.11) respectively correspond to the three phenomena listed above, in this order. A crucial point to make is that, in this right-hand side, all differential operators acting on $\psi$ are related to the variable $\boldsymbol{r}$ of the configuration space, not of the ambient physical space. In contrast, the gradient of the left-hand side is the usual transport term in the physical space $\boldsymbol{u} \cdot \nabla_{\boldsymbol{x}}$. In the absence of such a transport term (this will indeed the case for extremely simple geometries, such as that of a Couette flow), (3.11) is simply a family of Fokker-Planck equation posed in variables $(t, \boldsymbol{r})$ and parameterized in $\boldsymbol{x}$. These equations only speak to one another through the macroscopic field $\boldsymbol{u}$. When the transport term is present, (3.11) is a genuine partial differential equation in all variables $(t, \boldsymbol{x}, \boldsymbol{r})$. It is intuitively clear that the latter case is much more difficult, computationally and mathematically.

Once $\psi$ is obtained, we need to formalize its contribution to the total stress, and, further, its impact on the macroscopic flow.

Let us return to some basics of continuum mechanics. When defining the stress tensor, the commonly used mental image is the following: consider the material, cut it by a planar section into two pieces, try and separate the pieces. The reaction force experienced when separating the two pieces is $\boldsymbol{\tau} \boldsymbol{n}$, where $\boldsymbol{\tau}$ is the stress tensor and $\boldsymbol{n}$ the unit vector normal to the cut plane. Varying the orientation of the cut planes, and thus $\boldsymbol{n}$, provides all the entries of $\tau$. Applying the same 'methodology' to the polymeric fluid under consideration gives rise to two contributions (see Figure 8): that, usually considered, of the solvent, which contributes as the usual Newtonian stress tensor, and that coming from all the polymeric chains reacting. The latter needs to be evaluated quantitatively. This is the purpose of the 
so-called Kramers formula.

$$
\boldsymbol{\tau}_{p}(t, \boldsymbol{x})=-n_{p} k T \operatorname{Id}+n_{p} \int(\boldsymbol{r} \otimes \boldsymbol{F}(\boldsymbol{r})) \psi(t, \boldsymbol{x}, \boldsymbol{r}) d \boldsymbol{r},
$$

where $\otimes$ denotes the tensor product $\left(\boldsymbol{r} \otimes \boldsymbol{F}(\boldsymbol{r})\right.$ is a matrix with $(i, j)$-component $\left.\boldsymbol{r}_{i} \boldsymbol{F}_{j}(\boldsymbol{r})\right)$ and $n_{p}$ denotes the total number of polymeric chain per unit volume. Note that the first term only changes the pressure by an additive constant.

The complete system of equation combines the equation of conservation of momentum at the macroscopic level, the incompressibility constraint, the Kramers formula, and the Fokker-Planck equation for the distribution of the end-to-end vector:

$$
\left\{\begin{array}{l}
\rho\left(\frac{\partial \boldsymbol{u}}{\partial t}+(\boldsymbol{u} \cdot \nabla) \boldsymbol{u}\right)-\eta \Delta \boldsymbol{u}+\nabla p-\operatorname{div} \boldsymbol{\tau}_{p}=\rho \boldsymbol{f}, \\
\operatorname{div} \boldsymbol{u}=0 \\
\boldsymbol{\tau}_{p}(t, \boldsymbol{x})=n_{p} \int(\boldsymbol{r} \otimes \boldsymbol{F}(\boldsymbol{r})) \psi(t, \boldsymbol{x}, \boldsymbol{r}) d \boldsymbol{r}-n_{p} k T \mathrm{Id}, \\
\frac{\partial \psi(t, \boldsymbol{x}, \boldsymbol{r})}{\partial t}+\boldsymbol{u}(t, \boldsymbol{x}) \cdot \nabla_{\boldsymbol{x}} \psi(t, \boldsymbol{x}, \boldsymbol{r}) \\
\quad=-\operatorname{div}_{\boldsymbol{r}}\left(\left(\nabla_{\boldsymbol{x}} \boldsymbol{u}(t, \boldsymbol{x}) \boldsymbol{r}-\frac{2}{\zeta} \boldsymbol{F}(\boldsymbol{r})\right) \psi(t, \boldsymbol{x}, \boldsymbol{r})\right)+\frac{2 k T}{\zeta} \Delta_{\boldsymbol{r}} \psi(t, \boldsymbol{x}, \boldsymbol{r}) .
\end{array}\right.
$$

For future reference, let us rewrite this system of equations in a non-dimensional form (see Section 4.3 and 4.45) for the derivation of the non-dimensional equations and the definition of the non-dimensional numbers Re, $\epsilon$ and We ):

$$
\left\{\begin{array}{l}
\operatorname{Re}\left(\frac{\partial \boldsymbol{u}}{\partial t}+(\boldsymbol{u} \cdot \nabla) \boldsymbol{u}\right)-(1-\epsilon) \Delta \boldsymbol{u}+\nabla p-\operatorname{div} \boldsymbol{\tau}_{p}=\boldsymbol{f}, \\
\operatorname{div} \boldsymbol{u}=0, \\
\boldsymbol{\tau}_{p}(t, \boldsymbol{x})=\frac{\epsilon}{\mathrm{We}}\left(\int(\boldsymbol{r} \otimes \boldsymbol{F}(\boldsymbol{r})) \psi(t, \boldsymbol{x}, \boldsymbol{r}) d \boldsymbol{r}-\mathrm{Id}\right), \\
\frac{\partial \psi(t, \boldsymbol{x}, \boldsymbol{r})}{\partial t}+\boldsymbol{u}(t, \boldsymbol{x}) \cdot \nabla_{\boldsymbol{x}} \psi(t, \boldsymbol{x}, \boldsymbol{r}) \\
\quad=-\operatorname{div}_{\boldsymbol{r}}\left(\left(\nabla_{\boldsymbol{x}} \boldsymbol{u}(t, \boldsymbol{x}) \boldsymbol{r}-\frac{1}{2 \mathrm{We}} \boldsymbol{F}(\boldsymbol{r})\right) \psi(t, \boldsymbol{x}, \boldsymbol{r})\right)+\frac{1}{2 \mathrm{We}} \Delta_{\boldsymbol{r}} \psi(t, \boldsymbol{x}, \boldsymbol{r}) .
\end{array}\right.
$$

The multiscale nature of this system is obvious. In the specific context of complex fluids, such a system is called a micro-macro model. It is equally obvious on (3.14) that the computational task will be demanding. Formally, system (3.14) couples a Navier-stokes type equation (that is, an equation the simulation of which is one of the major challenge of scientific computing, and has been the topic of thousands of year/researchers efforts), and, 


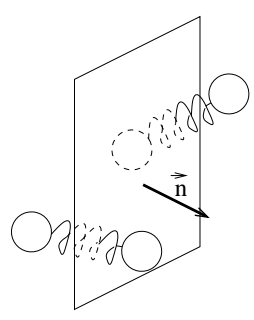

Figure 8: Kramer Formula : the contribution of all polymeric chains to the stress is obtained summing over all chains cut by the plane considered.

at each point (that is, slightly anticipating on the discretization, at each node of the mesh used for the space discretization of the macroequation), one parabolic partial differential equation set on the space of $\boldsymbol{r}$. It is thus intuitively clear that, in nature, such a micromacro strategy will be limited to as simple as possible test cases. We will return to this later.

With a view to generalizing the approach followed above to various other contexts, it is interesting to write system (3.14) as a particular form of a more abstract system. A purely macroscopic description of non-Newtonian fluids, issued from equations of the type (2.13) - 2.15) typically reads:

$$
\left\{\begin{array}{l}
\frac{D \boldsymbol{u}}{D t}=\mathcal{F}\left(\boldsymbol{\tau}_{p}, \boldsymbol{u}\right) \\
\frac{D \boldsymbol{\tau}_{p}}{D t}=\mathcal{G}\left(\boldsymbol{\tau}_{p}, \boldsymbol{u}\right)
\end{array}\right.
$$

In contrast, a multiscale approach introduces an additional intermediate step, where the stress tensor is calculated as an average value of a field $\Sigma$ describing the microstructure. An evolution equation is written on the latter:

$$
\left\{\begin{aligned}
\frac{D \boldsymbol{u}}{D t} & =\mathcal{F}\left(\boldsymbol{\tau}_{p}, \boldsymbol{u}\right) \\
\boldsymbol{\tau}_{p} & =\text { average over } \Sigma \\
\frac{D \Sigma}{D t} & =\mathcal{G}_{\mu}(\Sigma, \boldsymbol{u}) .
\end{aligned}\right.
$$

The structure of system (3.16) is a common denominator to all multiscale models for complex fluids. Beyond this, it also illustrates the nature of all multiscale approaches, in very different contexts (see C. Le Bris [77]). A global macroscopic equation is coupled with a local (microscopic) equation, via an averaging formula. For instance, the reader familiar with homogenization theory for materials recognizes in (3.16) the homogenized equation, the value of the homogenized tensor, and the corrector equation, respectively. On the numerical front, it is also a structure shared with multiscale algorithmic approaches: a global coarse 
solver coupled to a local fine one using an averaging process (think to the Godunov scheme for solving the Riemann problem in computational fluid dynamics). 


\section{The stochastic approach}

We now need to complement the derivation of the previous section in three directions:

- We need to introduce a definite stochastic description of the polymeric chain that will justify the expressions employed for the elongation force and the Brownian force in (3.11).

- We need to provide other entropic forces, alternative to the simple Hookean force (3.10).

- We need to prepare for an efficient computational strategy that allows for the practical simulation of systems of the type (3.14) even when the configuration space for the chain is high-dimensional.

For all three aspects, stochastic analysis comes into play. This is why we devote the next section to a brief introduction of the major ingredients from stochastic analysis needed in the sequel. Such ingredients are traditionally not necessarily well known by readers more familiar with the classical analysis of partial differential equations and their discretization techniques in the engineering sciences (finite element methods, etc...). Of course, the reader already familiar with the basics of stochastic analysis may easily skip the next section and directly proceed to Section 4.2 In any event, Subsection 4.1 is no more than a surrogate for a more comprehensive course of Stochastic Analysis, as contained in the classical textbooks F. Comets and T. Meyre [26, I. Karatzas and S.E. Shreve [69], P.E. Kloeden and E. Platen [73, B. Oksendal [100, L.C.G. Rogers and D. Williams [113, 114, D. Revuz and M. Yor [112], D. Stroock and S.R.S. Varadhan [116]. We also refer to D.J. Higham [58] for an attractive practical initiation.

\subsection{Initiation to Stochastic Differential Equations}

We assume that the reader is familiar with the following elementary notions of Probability Theory: the notion of probability space $(\Omega, \mathcal{A}, \mathbb{P})$, where $\Omega$ is the space, $\mathcal{A}$ is a $\sigma$-algebra, and $\mathbb{P}$ is the probability measure that equips the space; the notion of vector-valued or scalarvalued random variables defined on this probability space; the notion of expectation value and the notion of law.

A rather abstract notion we must define before getting to the heart of the matter is the notion of filtration: a filtration $\left(\mathcal{F}_{t}, t \geq 0\right)$ is an increasing sequence, indexed by time $t \in \mathbb{R}_{+}$, of subsets of the $\sigma$-algebra $\mathcal{A}$. The filtration $\mathcal{F}_{t}$ is to be thought of as the set of information available at time $t$.

\subsubsection{The Monte Carlo method}

The Monte Carlo method is a stochastic method to compute the expectation value of a random variable. Let $X$ be a random variable with finite variance:

$$
\operatorname{Var}(X)=\mathbb{E}\left((X-\mathbb{E}(X))^{2}\right)=\mathbb{E}\left(X^{2}\right)-(\mathbb{E}(X))^{2}<\infty .
$$


The principle of the Monte Carlo method is to approximate the expectation value $\mathbb{E}(X)$ by the empirical mean

$$
I_{K}=\frac{1}{K} \sum_{k=1}^{K} X^{k}
$$

where $\left(X^{k}\right)_{k \geq 0}$ are independent identically distributed (i.i.d.) random variables with law the law of $X$.

The foundation of the Monte Carlo method is based on two mathematical results. The law of large numbers states that (if $\mathbb{E}|X|<\infty$ )

$$
\text { almost surely, } \lim _{K \rightarrow \infty} I_{K}=\mathbb{E}(X) \text {. }
$$

The central limit theorem gives the rate of convergence (if $\left.\mathbb{E}\left((X)^{2}\right)<\infty\right): \forall a>0$,

$$
\lim _{K \rightarrow \infty} \mathbb{P}\left(\left|I_{K}-\mathbb{E}(X)\right| \leq a \sqrt{\frac{\operatorname{Var}(X)}{K}}\right)=\frac{1}{\sqrt{2 \pi}} \int_{-a}^{a} \exp \left(-x^{2} / 2\right) d x
$$

This estimate enables to build a posteriori error estimates (so-called confidence intervals) by choosing typically $a=1.96$ so that $\frac{1}{\sqrt{2 \pi}} \int_{-a}^{a} \exp \left(-x^{2} / 2\right) d x=95 \%$, and estimating the variance $\operatorname{Var}(X)$ by the empirical variance

$$
V_{K}=\frac{1}{K} \sum_{k=1}^{K}\left(X^{k}\right)^{2}-\left(I_{K}\right)^{2} .
$$

This estimate shows that the rate of convergence of a Monte Carlo method is of order $\sqrt{\frac{\operatorname{Var}(X)}{K}}$ : to reduce the error, one needs to add more replicas (increase $K$ ), or reduce the variance of the random variable (which is the basis of variance reduction methods, see Section 5.4 below).

\subsubsection{Brownian motion and simple stochastic differential equations}

Let us now introduce the notion of a (continuous-in-time) stochastic process, as a family of random variables $\left(X_{t}\right)_{t \geq 0}$ indexed by time $t \in \mathbb{R}_{+}$. Given a stochastic process $X_{t}$, we may consider the natural filtration generated by $X_{t}$, that is the filtration $\mathcal{F}_{t}$ formed, for each $t$, by the smallest $\sigma$-algebra for which the maps $\omega \longrightarrow X_{s}(\omega), 0 \leq s \leq t$, are measurable functions.

Conversely, being given a filtration $\mathcal{F}_{t}$, a stochastic process such that, for all $t, X_{t}$ is a measurable function with respect to $\mathcal{F}_{t}$, is called a $\mathcal{F}_{t^{-}}$adapted stochastic process.

A remarkable random process is the Brownian motion, which we now briefly introduce.

The formal motivation for the introduction of the Brownian motion is the need for modelling random trajectories. For such trajectories, the random perturbations at time $t$ 
should be independent of those at time $t^{\prime}<t$, and essentially the same. By this we mean that the two should share the same law. The mathematical manner to formalize the above somewhat vague object is the notion of Brownian motion. There are several ways to define a Brownian motion. One way is to take the limit of random walks on lattices, with an adequate scaling law on the size of the lattice and time. The definition we choose to give here is the axiomatic definition. We define a Brownian motion as a real-valued random process enjoying the following three properties. First, its trajectories, that is, the maps $s \longrightarrow X_{s}(\omega)$ are continuous, for almost all $\omega \in \Omega$. Second, it has stationary increments, that is, when $s \leq t$, the random variable $X_{t}-X_{s}$ is independent from the $\sigma$-algebra $\mathcal{F}_{s}$ : otherwise stated, for all $A \in \mathcal{F}_{s}$, and all bounded measurable function $f, \mathbb{E}\left(1_{A} f\left(X_{t}-X_{s}\right)\right)=\mathbb{E}\left(f\left(X_{t}-X_{s}\right)\right) \mathbb{P}(A)$. Third, it has stationary increments, that is when $s \leq t, X_{t}-X_{s}$ and $X_{t-s}-X_{0}$ share the same law. In fact, the conjunction of these three properties implies that, necessarily, $X_{t}-X_{0}$ is a Gaussian variable, with mean $r t$ (for some $r$ ) and variance $\sigma^{2} t$ (for some $\sigma$ ). When $r=0$ and $\sigma=1$, the Brownian motion is called a standard Brownian motion.

We now wish to define differential equations, typically modelling the motion of particles, which are subject to random perturbations. The adequate mathematical notion for this purpose is that of stochastic differential equations. Let us fix a probability space $(\Omega, \mathcal{A}, \mathbb{P})$, where it is sometimes useful to think of $\Omega$ as the product $\Omega=\Omega_{1} \times \Omega_{2}$ where $\Omega_{1}$ models the randomness due to the initial condition supplied for the differential equation, and $\Omega_{2}$ models the randomness associated with the perturbations occurring at all positive times.

Let us also consider a $\sigma$-field $\mathcal{A}$, a filtration $\mathcal{F}_{t}$, and a $\mathcal{F}_{t}$-adapted Brownian motion $B_{t}$. Let $\sigma>0$ denote a fixed parameter, called diffusion, and $b(t, x)$ a fixed regular function, called drift. As regards regularity issues, the most appropriate setting is to consider functions $b$ measurable with respect to time $t$, Lipschitz with respect to the space variable $x$, and with a growth at most linear at infinity, that is $|f(t, x)| \leq C(1+|x|)$ for all $t, x$. For simplicity, the Lipschitz constant and the growth constant are assumed uniform on $t \in[0, T]$. We then define the stochastic differential equation:

$$
d X_{t}=b\left(t, X_{t}\right) d t+\sigma d B_{t},
$$

with initial condition $X_{0}\left(\omega_{1}\right)$. Equation (4.1) is formal. It is to be understood in the following sense: $X_{t}$ is said a solution to (4.1) when

$$
X_{t}\left(\omega_{1}, \omega_{2}\right)=X_{0}\left(\omega_{1}\right)+\int_{0}^{t} b\left(s, X_{s}\left(\omega_{1}, \omega_{2}\right)\right) d s+\sigma B_{t}\left(\omega_{2}\right),
$$

almost surely. Our setting in (4.1)-(4.2) is one dimensional, but the notion is readily extended to the higher dimensional context (see (4.12) below).

Note that we do not question here the existence and the uniqueness of the solutions to the above stochastic differential equations. This is beyond the scope of this simplified presentation. Let us only say that we assume for the rest of this expository survey that typically the Lipschitz regularity mentioned above is sufficient to define in a unique manner the solution to (4.1). For less regular drifts and related questions, we refer the interested 
reader to Section [6] The modelling of complex fluids may indeed naturally involve non regular drifts.

\subsubsection{Stochastic integration}

The above form (4.1) is actually a simple form of a stochastic differential equations. This form is sufficient to deal with the context of flexible polymers, which is the main topic of this presentation. However, for rigid polymers, briefly addressed in Section [7] and some other types of complex fluids, it is useful to define the general form of stochastic differential equations. This is the purpose of this short section.

In addition, the consideration of this general form of stochastic differential equation will allow us to introduce a technical lemma which will be crucially useful, even in our simple setting.

Using a standard Brownian motion $B_{t}$, the Itô integral may be constructed. The construction of this notion of integral is similar to that of the Riemann integral, proceeding first for piecewise constant functions, and then generalizing the notion to more general functions by approximation. Consider a decomposition $\left\{s_{0}=0, \ldots, s_{j}, \ldots, s_{n}=t\right\}$ of the range $[0, t]$ and a piecewise constant process

$$
Y_{s}(\omega)=\sum_{j=1}^{n} \tilde{Y}_{j-1}(\omega) 1_{\left.s_{j-1}, s_{j}\right]}(s)
$$

constructed from random variables $\tilde{Y}_{j}$ (such that $\mathbb{E}\left(\left|\tilde{Y}_{j}\right|\right)<+\infty$ ). Then we define

$$
\int_{0}^{t} Y_{s} d B_{s}=\sum_{j=1}^{n} \tilde{Y}_{j-1}\left(B_{s_{j}}-B_{s_{j-1}}\right) .
$$

Next, for any arbitrary stochastic process $Y_{t}(\omega)$ such that, almost surely,

$\int_{0}^{T} Y_{t}(\omega)^{2} d t<+\infty$, this allows, by approximation, for the definition of the stochastic pro-

$$
\int_{0}^{t} Y_{s} d B_{s}
$$

In the simple case when $Y_{t} \equiv 1$, this coincides with the already known notion $\int_{0}^{t} d B_{s}=B_{t}$. Notice that by taking the expectation of 4.3 , we have, for all $t \in[0, T]$

$$
\mathbb{E}\left(\int_{0}^{t} Y_{s} d B_{s}\right)=0
$$

which actually holds (by an approximation argument) for any arbitrary stochastic process $Y_{t}$ such that $\mathbb{E}\left(\int_{0}^{T} Y_{t}(\omega)^{2} d t\right)<+\infty$. 
Having defined the Itô integral, we are in position, for any regular drift $b$ and diffusion $\sigma$, to define the stochastic differential equation:

$$
d X_{t}=b\left(t, X_{t}\right) d t+\sigma\left(t, X_{t}\right) d B_{t},
$$

supplied with the initial condition $X_{0}$. Mathematically:

$$
X_{t}\left(\omega_{1}, \omega_{2}\right)=X_{0}\left(\omega_{1}\right)+\int_{0}^{t} b\left(s, X_{s}\left(\omega_{1}, \omega_{2}\right)\right) d s+\left(\int_{0}^{t} \sigma\left(s, X_{s}\right) d B_{s}\right)\left(\omega_{1}, \omega_{2}\right),
$$

almost surely. In the right-hand side, the first integral is the Lebesgue integral, the second one is a Itô integral.

\subsubsection{Itô calculus and Fokker Planck equation}

We now wish to relate the above stochastic differential equation 4.5 with a partial differential equation. The latter is indeed the Fokker-Planck equation

$$
\frac{\partial p}{\partial t}(t, x)+\frac{\partial}{\partial x}(b(t, x) p(t, x))-\frac{\partial^{2}}{\partial x^{2}}\left(\frac{\sigma^{2}(t, x)}{2} p(t, x)\right)=0 .
$$

In the context of deterministic equations, the reader is perhaps familiar with the intimate link between ordinary differential equations and linear transport equations. This is the famous method of characteristics, which we briefly recall here. Consider the linear transport equation

$$
\frac{\partial u}{\partial t}(t, x)-b(x) \frac{\partial u}{\partial x}(t, x)=0
$$

supplied with the initial condition $u_{0}$ at initial time. Its solution reads

$$
u(t, x)=u_{0}(X(t ; 0, x))
$$

where $X(t ; 0, x)$ is the solution at time $t$ of the ordinary differential equation

$$
\frac{d X(t)}{d t}=b(X(t))
$$

starting from the initial condition $X(0)=x$. The proof of this fact is elementary. For $s \in[0, t]$, we have (where $X(s)=X(s ; 0, x)$ )

$$
\begin{aligned}
\frac{\partial}{\partial s}(u(t-s, X(s))) & =-\frac{\partial u}{\partial t}(t-s, X(s))+\frac{d X}{d t}(s) \frac{\partial u}{\partial x}(t-s, X(s)), \\
& =-\frac{\partial u}{\partial t}(t-s, X(s))+b(X(s)) \frac{\partial u}{\partial x}(t-s, X(s))=0 .
\end{aligned}
$$

By integrating this relation from $s=0$ to $s=t$, we thus obtain [4.9). 
A similar type of argument, based on the so-called Feynman-Kac Formula would show the relation holding between the stochastic differential equation (4.5) and a partial differential equation, called the backward Kolmogorov equation. A dual viewpoint to the above one illustrates the relation between the stochastic differential equation (4.5) and the FokkerPlanck equation (4.7). We now present it.

First, we need to establish a chain rule formula in the context of stochastic processes. This is the purpose of the celebrated Itô formula (stated here in a simple one-dimensional setting).

Lemma 1 Itô Formula Let $X_{t}$ solve

$$
d X_{t}=b\left(t, X_{t}\right) d t+\sigma\left(t, X_{t}\right) d B_{t},
$$

in the sense of (4.6). Then, for all $C^{2}$ regular function $\varphi$,

$$
d \varphi\left(X_{t}\right)=\left(\varphi^{\prime}\left(X_{t}\right) b\left(t, X_{t}\right)+\frac{1}{2} \varphi^{\prime \prime}\left(X_{t}\right) \sigma\left(t, X_{t}\right)^{2}\right) d t+\varphi^{\prime}\left(X_{t}\right) \sigma\left(t, X_{t}\right) d B_{t}
$$

in the sense

$$
\begin{aligned}
\varphi\left(X_{t}\right)= & \varphi\left(X_{0}\right)+\int_{0}^{t}\left(\varphi^{\prime}\left(X_{s}\right) b\left(s, X_{s}\right)+\frac{1}{2} \varphi^{\prime \prime}\left(X_{s}\right) \sigma\left(s, X_{s}\right)^{2}\right) d s \\
& +\int_{0}^{t} \varphi^{\prime}\left(X_{s}\right) \sigma\left(s, X_{s}\right) d B_{s} .
\end{aligned}
$$

The point is of course to compare with the deterministic setting, corresponding to $\sigma=0$, and for which no second derivatives of $\varphi$ appears since

$$
\frac{d}{d t} \varphi\left(X_{t}\right)=\varphi^{\prime}\left(X_{t}\right) \frac{d X_{t}}{d t} .
$$

We are now in position to relate (4.5) and (4.7). Assume that all conditions of regularity are satisfied, which gives sense to the formal manipulations we now perform. Let us assume that $X_{0}$, the initial condition for (4.5) has law $p_{0}$, where $p_{0}$ is the initial condition given to (4.7). Let us denote by $p(t, x)$ the probability density (with respect to the Lebesgue measure) of the random variable $X_{t}$.

For any arbitrary $C^{2}$ function $\varphi$, we write

$$
\int \varphi(x) \frac{\partial p}{\partial t}(t, x) d x=\frac{d}{d t} \int \varphi(x) p(t, x) d x=\frac{d}{d t} \mathbb{E}\left(\varphi\left(X_{t}\right)\right) .
$$

Now, taking the expectation of 4.11), we obtain

$$
\begin{aligned}
\mathbb{E}\left(\varphi\left(X_{t}\right)\right) & =\mathbb{E}\left(\varphi\left(X_{0}\right)\right)+\mathbb{E}\left(\int_{0}^{t}\left(\varphi^{\prime}\left(X_{s}\right) b\left(s, X_{s}\right)+\frac{1}{2} \varphi^{\prime \prime}\left(X_{s}\right) \sigma\left(s, X_{s}\right)^{2}\right) d s\right) \\
& +\mathbb{E}\left(\int_{0}^{t} \varphi^{\prime}\left(X_{s}\right) \sigma\left(s, X_{s}\right) d B_{s}\right) .
\end{aligned}
$$


Under suitable regularity assumptions, the last term vanishes for all times (see (4.4)). We thus have

$$
\begin{aligned}
\int \varphi(x) \frac{\partial p}{\partial t}(t, x) d x & =\mathbb{E}\left(\varphi^{\prime}\left(X_{t}\right) b\left(t, X_{t}\right)+\frac{1}{2} \varphi^{\prime \prime}\left(X_{t}\right) \sigma\left(t, X_{t}\right)^{2}\right) \\
& =\int\left(\varphi^{\prime}(x) b(t, x)+\frac{1}{2} \varphi^{\prime \prime}(x) \sigma^{2}(t, x)\right) p(t, x) d x \\
& =\int \varphi(x)\left(-\frac{\partial}{\partial x}(p b)(t, x)+\frac{1}{2} \frac{\partial^{2}}{\partial x^{2}}\left(\sigma^{2} p\right)\right) d x
\end{aligned}
$$

This precisely shows that $p$ is the solution to (4.7), which starts from $p_{0}$ at initial time.

A similar argument, based on the multi-dimensional Itô Formula (a straightforward extension of Lemma 1), allows to establish the same correspondence between, on the one-hand, the vectorial stochastic differential equation

$$
d \boldsymbol{X}_{t}=\boldsymbol{b}\left(t, \boldsymbol{X}_{t}\right) d t+\boldsymbol{\sigma}\left(t, \boldsymbol{X}_{t}\right) d \boldsymbol{B}_{t}
$$

where $\boldsymbol{X}_{t}$ is a random process with values in $\mathbb{R}^{N}, \boldsymbol{b}(t, \cdot)$ is a vector field on $\mathbb{R}^{N}$ for all times, $\sigma$ is $N \times K$ matrix valued function, and $\boldsymbol{B}_{t}$ is a $K$-dimensional Brownian motion, and, on the other hand, the Fokker-Planck equation.

$$
\frac{\partial p}{\partial t}(t, \boldsymbol{x})+\operatorname{div}\left((\boldsymbol{b}(t, \boldsymbol{x}) p(t, \boldsymbol{x}))-\frac{1}{2} \nabla^{2}:\left(\boldsymbol{\sigma} \boldsymbol{\sigma}^{T} p\right)(t, \boldsymbol{x})=0\right.
$$

where $\nabla^{2}:\left(\boldsymbol{\sigma} \boldsymbol{\sigma}^{T} p\right)=\sum_{i, j=1}^{N} \frac{\partial^{2}}{\partial x_{i} \partial x_{j}}\left(\sum_{k=1}^{K} \sigma_{i, k} \sigma_{j, k} p\right)$.

Under appropriate conditions of regularity (which we have omitted to make precise above), we may therefore claim that the law of any process solving the stochastic differential equation solves the Fokker Planck equation. The converse assertion is false. Let us give the following simple illustration. Consider the stochastic differential equation

$$
d X_{t}=-\frac{1}{2} X_{t} d t+d B_{t}
$$

with initial condition $X_{0}$ normally distributed with zero mean and variance one (and independent of $B_{t}$ ), and the associated Fokker-Planck equation

$$
\frac{\partial p(t, x)}{\partial t}-\frac{1}{2} \frac{\partial}{\partial x}(x p(t, x))-\frac{1}{2} \frac{\partial^{2}}{\partial x^{2}} p(t, x)=0 .
$$

Clearly, the solution to (4.14 reads

$$
X_{t}=e^{-t / 2} X_{0}+\int_{0}^{t} e^{(s-t) / 2} d B_{s}
$$


Therefore, for all $t \geq 0, X_{t}$ is a Gaussian random variable with zero mean and variance one and of course, as the previous argument shows, $p(t, x)=\frac{1}{\sqrt{2 \pi}} \exp \left(-x^{2} / 2\right)$ indeed solves the Fokker-Planck equation (4.15). However, any random process $Y_{t}$ such that its marginals in time (namely the law of $Y_{t}$, for fixed $t$ ) are normally distributed with zero mean and variance one, such as the constant process $Y_{t}=X_{0}$, does not solve (4.14). The process encodes more information than the law of the time marginals, and it is thus intuitively clear that the knowledge of the law of the time marginals is not sufficient to know the trajectory of the process. Otherwise stated, knowing the law of the time marginals allows to compute all expectation values of the type $\mathbb{E}\left(\varphi\left(X_{t}\right)\right)$, but, e.g., not quantities such as $\mathbb{E}\left(\psi\left(X_{t}, X_{s}\right)\right)$.

Nevertheless, for most situations of interest, and in particular for many physically relevant situations, only the knowledge of expectation values such as $\mathbb{E}\left(\varphi\left(X_{t}\right)\right)$ is sufficient. In such situations, solving the Fokker-Planck equation, when it is practically feasible, provides all the information needed. In our context of the modelling of complex fluids, we can therefore equivalently use the stochastic differential viewpoint, or the Fokker-Planck viewpoint. Efficiency considerations indicate which is the best strategy, depending on the dimension of the problem at hand, and other parameters. We will return to this below.

\subsubsection{Discretization of SDEs}

We now briefly give here some basic elements of numerical analysis for stochastic differential equations. For this purpose, we assume that the reader is familiar with the discretization techniques for ordinary differential equations and the associated analysis (see E. Hairer, S.P. Norsett and G. Wanner [55, [56]).

For simplicity, we argue on the one-dimensional simple case (4.1), that is

$$
d X_{t}=b\left(t, X_{t}\right) d t+\sigma d B_{t}
$$

with in addition, a constant $\sigma$. We leave aside questions related to the general case $d X_{t}=$ $b\left(t, X_{t}\right) d t+\sigma\left(t, X_{t}\right) d B_{t}$, which, owing to the dependence of $\sigma$ upon the solution $X_{t}$, might be significantly more technical than the simple case considered here (see Remark 4.1 below). Likewise, we assume that $b$ is regular and that all questions of existence and uniqueness have been settled.

The crucial point to bear in mind is that, in contrast to the deterministic setting, there are two notions of convergence for a scheme discretizing a stochastic differential equation.

The notion of convergence analogous to the deterministic notion is:

Definition 1 The numerical scheme is said strongly convergent and is said to have strong order of convergence $\alpha>0$ when there exists a constant $C$, possibly depending on the interval of integration $[0, T]$, such that, for all timesteps $\Delta t$ and for all integer $n \in[0, T / \Delta t]$,

$$
\mathbb{E}\left(\left|\bar{X}_{n}-X_{t_{n}}\right|\right) \leq C(\Delta t)^{\alpha},
$$

where $X_{t_{n}}$ denotes the exact solution at time $t_{n}=n \Delta t$, and $\bar{X}_{n}$ denotes its numerical approximation. 

is:

A weaker notion, which is a better metric to assess convergence in practical situations,

Definition 2 Under the same conditions as the above definition, the scheme is said weakly convergent and is said to have weak order of convergence $\beta>0$ when for all integer $n \in$ $[0, T / \Delta t]$

$$
\left|\mathbb{E}\left(\varphi\left(\bar{X}_{n}\right)\right)-\mathbb{E}\left(\varphi\left(X_{t_{n}}\right)\right)\right| \leq C(\Delta t)^{\beta},
$$

for all $C^{\infty}$ function $\varphi$, with polynomial growth at infinity, and such that all its derivatives also have polynomial growth at infinity.

The latter definition, specific to the stochastic setting, is motivated by the fact that in many applications, as already mentioned above, the stochastic differential equation is simulated only to evaluate some expectation values $\mathbb{E}\left(\varphi\left(X_{t}\right)\right)$. This will be the case for complex fluid flows simulation (see the expression (4.30) of the stress tensor below). The notion of weak convergence is tailored for this purpose. In contrast to the strong convergence, it does not measure the accuracy of the approximation of each realization (each "trajectory") (note indeed that $\mathbb{P}\left(\left|\bar{X}_{n}-X_{t_{n}}\right| \geq a\right) \leq \frac{1}{a} \mathbb{E}\left(\left|\bar{X}_{n}-X_{t_{n}}\right|\right)$ ), but only the accuracy of the mean. Of course, strong convergence clearly implies weak convergence.

Let us now mention the simplest possible scheme for the numerical integration of [4.5). It is the forward (or explicit) Euler scheme:

$$
\bar{X}_{n+1}=\bar{X}_{n}+b\left(t_{n}, \bar{X}_{n}\right) \Delta t+\sigma\left(B_{t_{n+1}}-B_{t_{n}}\right) .
$$

Since the increment $B_{t_{n+1}}-B_{t_{n}}$ is a centered Gaussian random variable with variance $t_{n+1}-t_{n}=\Delta t$, the scheme also writes

$$
\bar{X}_{n+1}=\bar{X}_{n}+b\left(t_{n}, \bar{X}_{n}\right) \Delta t+\sigma \sqrt{\Delta t} G_{n},
$$

where $\left(G_{n}\right)_{n \geq 0}$ denote i.i.d. standard normal random variables.

It is easy to see that the scheme (4.18) arises from the approximation

$$
\begin{aligned}
X_{t_{n+1}}-X_{t_{n}} & =\int_{t_{n}}^{t_{n+1}} b\left(t, X_{t}\right) d t+\sigma \int_{t_{n}}^{t_{n+1}} d B_{t}, \\
& \approx b\left(t_{n}, X_{t_{n}}\right) \Delta t+\sigma\left(B_{t_{n+1}}-B_{t_{n}}\right) .
\end{aligned}
$$

The second integration in the right-hand side being exact, the precision order is exactly that of the approximation of the Lebesgue integral, and is therefore $\alpha=1$. This is the strong order of convergence, and we leave to the reader the task to check this is also the weak order of convergence.

Remark 4.1 Actually, the above argument is slightly misleading. It is specific to the case of a constant diffusion $\sigma$ as in (4.1) or, more appropriately stated, to a deterministic diffusion 
$\sigma$ that may depend on time, but that does not depend on the solution $X_{t}$. When the latter depends on the solution, that is

$$
d X_{t}=b\left(t, X_{t}\right) d t+\sigma\left(X_{t}\right) d B_{t} .
$$

then the Euler scheme

$$
\bar{X}_{n+1}=\bar{X}_{n}+b\left(t_{n}, \bar{X}_{n}\right) \Delta t+\sigma\left(\bar{X}_{n}\right)\left(B_{t_{n+1}}-B_{t_{n}}\right)
$$

(actually also called the Euler-Maruyama scheme) is only of strong order $\alpha=1 / 2$, but it remains of weak order $\beta=1$. The reason lies in the difference between the Itô calculus and the usual deterministic calculus. In fact, to obtain strong convergence with order 1 , the adequate scheme to employ (at least for one-dimensional processes) is the Euler-Milstein scheme:

$$
\begin{aligned}
\bar{X}_{n+1}-\bar{X}_{n}= & b\left(t_{n}, \bar{X}_{n}\right) \Delta t+\sigma\left(\bar{X}_{n}\right)\left(B_{t_{n+1}}-B_{t_{n}}\right) \\
& +\frac{1}{2} \sigma\left(\bar{X}_{n}\right) \sigma^{\prime}\left(\bar{X}_{n}\right)\left(\left(B_{t_{n+1}}-B_{t_{n}}\right)^{2}-\Delta t\right) .
\end{aligned}
$$

It is of strong order of convergence $\alpha=1$, and of course agrees with the Euler-Maruyama scheme when $\sigma$ is independent of $X_{t}$.

\subsection{Back to the modelling}

Given the notions of the previous section, we are now in position to return to some key issues in the modelling step, briefly addressed earlier in Section 3 Our purpose there was only to concentrate on the multiscale problem, and reach as soon as possible a prototypical form of such a system. This has been performed with (3.14) at the price of some simplifications and shortcuts. Let us now take a more pedestrian approach to the problem, and dwell into some issues, based on our present mathematical knowledge of the stochastic formalism.

\subsubsection{The microscopic equation of motion}

Let us first concentrate on the two forces exerted by the solvent onto the chain, namely the friction force elongating the chain and the Brownian force modeling collisions.

For this purpose, we isolate one single bead, denote by $m$ its mass, $\boldsymbol{V}_{t}$ its velocity, and write the following equation of motion, called the Langevin equation:

$$
m d \boldsymbol{V}_{t}=-\zeta \boldsymbol{V}_{t} d t+D d \boldsymbol{B}_{t},
$$

where $\boldsymbol{B}_{t}$ denotes a standard, $d$-dimensional, Brownian motion and $D$ a scalar parameter to be determined. The solution of (4.22) is a so-called Ornstein-Uhlenbeck process:

$$
\boldsymbol{V}_{t}=\boldsymbol{V}_{0} \exp \left(-\frac{\zeta}{m} t\right)+\frac{D}{m} \int_{0}^{t} \exp \left(-\frac{\zeta}{m}(t-s)\right) d \boldsymbol{B}_{s}
$$


where $\boldsymbol{V}_{0}$ is the initial velocity, assumed independent of $\boldsymbol{B}_{t}$. Consequently, $\boldsymbol{V}_{t}$ is a Gaussian process with mean

$$
\mathbb{E}\left(\boldsymbol{V}_{t}\right)=\mathbb{E}\left(\boldsymbol{V}_{0}\right) \exp \left(-\frac{\zeta}{m} t\right),
$$

and covariance matrix

$$
\begin{aligned}
\mathbb{E}\left(\left(\boldsymbol{V}_{t}-\mathbb{E}\left(\boldsymbol{V}_{t}\right)\right) \otimes\left(\boldsymbol{V}_{t}-\mathbb{E}\left(\boldsymbol{V}_{t}\right)\right)\right) \\
=\mathbb{E}\left(\left(\boldsymbol{V}_{0}-\mathbb{E}\left(\boldsymbol{V}_{0}\right)\right) \otimes\left(\boldsymbol{V}_{0}-\mathbb{E}\left(\boldsymbol{V}_{0}\right)\right)\right) \exp \left(-\frac{2 \zeta}{m} t\right) \\
\quad+\frac{D^{2}}{2 \zeta m}\left(1-\exp \left(-\frac{2 \zeta}{m} t\right)\right) \mathrm{Id} .
\end{aligned}
$$

For the above derivation, we have assumed that the fluid is at rest. The process $\boldsymbol{V}_{t}$ is thus expected to be stationary, which imposes:

$$
\left\{\begin{array}{l}
\mathbb{E}\left(\boldsymbol{V}_{t}\right)=\mathbb{E}\left(\boldsymbol{V}_{0}\right)=0, \\
\mathbb{E}\left(\boldsymbol{V}_{t} \otimes \boldsymbol{V}_{t}\right)=\mathbb{E}\left(\boldsymbol{V}_{0} \otimes \boldsymbol{V}_{0}\right)=\frac{D^{2}}{2 \zeta m} \mathrm{Id} .
\end{array}\right.
$$

Using the principle of equipartition of energy, the mean kinetic energy $\frac{1}{2} m \mathbb{E}\left(\left\|\boldsymbol{V}_{t}\right\|^{2}\right)$ should be equal to $\frac{d}{2} k T$ thus the Nernst-Einstein relation:

$$
D=\sqrt{2 k T \zeta} .
$$

Let us next consider two beads, forming a dumbbell. We denote by $\boldsymbol{X}_{t}^{i}$ the (random) position of bead $i, i=1,2$, and $\boldsymbol{X}_{t}=\boldsymbol{X}_{t}^{2}-\boldsymbol{X}_{t}^{1}$ the end-to-end vector (see Figure 9). We also denote $\boldsymbol{R}=\frac{1}{2}\left(\boldsymbol{X}^{1}+\boldsymbol{X}^{2}\right)$ the position of the center of mass. In addition to the above two forces experienced by each of the beads, a force $\boldsymbol{F}\left(\boldsymbol{X}_{t}\right)$ of entropic nature is to be accounted for. We now know this well (see Section 3.1.2.

The Langevin equations for this simple two particle system reads:

$$
\left\{\begin{array}{l}
m d\left(\frac{d \boldsymbol{X}_{t}^{1}}{d t}\right)=-\zeta\left(\frac{d \boldsymbol{X}_{t}^{1}}{d t}-\boldsymbol{u}\left(t, \boldsymbol{X}_{t}^{1}\right)\right) d t+\boldsymbol{F}\left(\boldsymbol{X}_{t}\right) d t+\sqrt{2 k T \zeta} d \boldsymbol{B}_{t}^{1}, \\
m d\left(\frac{d \boldsymbol{X}_{t}^{2}}{d t}\right)=-\zeta\left(\frac{d \boldsymbol{X}_{t}^{2}}{d t}-\boldsymbol{u}\left(t, \boldsymbol{X}_{t}^{2}\right)\right) d t-\boldsymbol{F}\left(\boldsymbol{X}_{t}\right) d t+\sqrt{2 k T \zeta} d \boldsymbol{B}_{t}^{2}
\end{array}\right.
$$

where $\boldsymbol{B}_{t}^{1}$ and $\boldsymbol{B}_{t}^{2}$ are two independent, $d$-dimensional Brownian motions. In the limit of a vanishing $\frac{m}{\zeta}$, (that is when the characteristic timescale of relaxation to equilibrium for the end-to-end vector is far larger that this value), we obtain by linear combination of the above two Langevin equations:

$$
\left\{\begin{array}{l}
d \boldsymbol{X}_{t}=\left(\boldsymbol{u}\left(t, \boldsymbol{X}_{t}^{2}\right)-\boldsymbol{u}\left(t, \boldsymbol{X}_{t}^{1}\right)\right) d t-\frac{2}{\zeta} \boldsymbol{F}\left(\boldsymbol{X}_{t}\right) d t+2 \sqrt{\frac{k T}{\zeta}} d \boldsymbol{W}_{t}^{1}, \\
d \boldsymbol{R}_{t}=\frac{1}{2}\left(\boldsymbol{u}\left(t, \boldsymbol{X}_{t}^{1}\right)+\boldsymbol{u}\left(t, \boldsymbol{X}_{t}^{2}\right)\right) d t+\sqrt{\frac{k T}{\zeta}} d \boldsymbol{W}_{t}^{2}
\end{array}\right.
$$




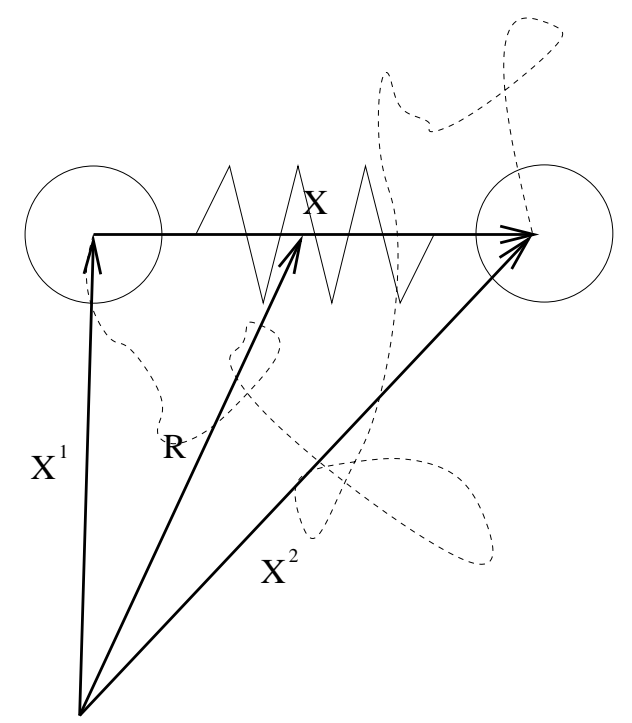

Figure 9: The dumbbell model: the end-to-end vector $\boldsymbol{X}$ is the vector connecting the two beads, while $\boldsymbol{R}$ gives the position of the center of mass.

where $\boldsymbol{W}_{t}^{1}=\frac{1}{\sqrt{2}}\left(\boldsymbol{B}_{t}^{2}-\boldsymbol{B}_{t}^{1}\right)$ and $\boldsymbol{W}_{t}^{2}=\frac{1}{\sqrt{2}}\left(\boldsymbol{B}_{t}^{1}+\boldsymbol{B}_{t}^{2}\right)$ are also two independent, $d$-dimensional Brownian motions. We assume they do not depend on space.

At this stage, the following assumptions are in order:

- as the length of the polymer is in any case far smaller than the spatial variations of the velocity of the solvent, we may perform the Taylor expansion

$$
\boldsymbol{u}\left(t, \boldsymbol{X}_{t}^{i}\right) \simeq \boldsymbol{u}\left(t, \boldsymbol{R}_{t}\right)+\nabla \boldsymbol{u}\left(t, \boldsymbol{R}_{t}\right)\left(\boldsymbol{X}_{t}^{i}-\boldsymbol{R}_{t}\right)
$$

for $i=1,2$,

- as $\frac{1}{2}\left(\boldsymbol{u}\left(t, \boldsymbol{X}_{t}^{1}\right)+\boldsymbol{u}\left(t, \boldsymbol{X}_{t}^{2}\right)\right) d t$ is of macroscopic size, in comparison to the microscopic variation $\sqrt{\frac{k T}{\zeta}} d \boldsymbol{W}_{t}^{2}$, the noise $\boldsymbol{W}_{t}^{2}=0$ is neglected.

Denoting by $\boldsymbol{W}_{t}=\boldsymbol{W}^{1}$, we obtain:

$$
\left\{\begin{aligned}
d \boldsymbol{X}_{t} & =\nabla \boldsymbol{u}\left(t, \boldsymbol{R}_{t}\right) \boldsymbol{X}_{t} d t-\frac{2}{\zeta} \boldsymbol{F}\left(\boldsymbol{X}_{t}\right) d t+2 \sqrt{\frac{k T}{\zeta}} d \boldsymbol{W}_{t}, \\
d \boldsymbol{R}_{t} & =\boldsymbol{u}\left(t, \boldsymbol{R}_{t}\right) d t .
\end{aligned}\right.
$$

The above system is supplied with initial conditions $\boldsymbol{X}_{0}$ and $\boldsymbol{R}_{0}$. The processes $\boldsymbol{X}_{t}$ and $\boldsymbol{W}_{t}$ are naturally indexed by the trajectories of fluid particles. The Eulerian description corre- 
sponding to the above Lagrangian description reads, for $\boldsymbol{X}_{t}(\boldsymbol{x})$ denoting the conformation at $\boldsymbol{x}$ at time $t$ :

$$
d \boldsymbol{X}_{t}(\boldsymbol{x})+\boldsymbol{u}(t, \boldsymbol{x}) \cdot \nabla \boldsymbol{X}_{t}(\boldsymbol{x}) d t=\nabla \boldsymbol{u}(t, \boldsymbol{x}) \boldsymbol{X}_{t}(\boldsymbol{x}) d t-\frac{2}{\zeta} \boldsymbol{F}\left(\boldsymbol{X}_{t}(\boldsymbol{x})\right) d t+2 \sqrt{\frac{k T}{\zeta}} d \boldsymbol{W}_{t} .
$$

Equation (4.29) is simply the stochastic version of the model already introduced in Section 3 under the form of equation (3.11). Indeed, the latter is the Fokker Planck associated to the stochastic differential (4.29). The function $\psi$ solution to (3.11) is the probability density of $\boldsymbol{X}_{t}(\boldsymbol{x})$ solution to (4.29). We refer the reader to the previous section for more details on the ingredient of stochastic analysis needed for the proof of this fact (see Section 4.1.4).

\subsubsection{The stress tensor}

Using the definition of the stress tensor recalled in Section 3 the Kramers formula can be shown. In the stochastic language we adopt here, it reads

$$
\boldsymbol{\tau}_{p}(t)=n_{p}\left(\mathbb{E}\left(\boldsymbol{X}_{t} \otimes \boldsymbol{F}\left(\boldsymbol{X}_{t}\right)\right)-k T \mathrm{Id}\right),
$$

where $\otimes$ denotes the tensorial product and $n_{p}$ is the concentration of polymers. See H.C. Öttinger [101, pp158-159], M. Doi and S.F. Edwards [36, section 3.7.4], R.B. Bird et al [12, section 13.3]. Of course, this expression is similar, in terms of $\boldsymbol{X}_{t}$, of the expression previously found on the law $\psi$ of $\boldsymbol{X}_{t}$, namely (3.12) in Section 3.

Using Itô calculus, an interesting alternative expression can be found for the stress tensor. Indeed, introducing the so-called structure tensor $\boldsymbol{X}_{t}(\boldsymbol{x}) \otimes \boldsymbol{X}_{t}(\boldsymbol{x})$, we have:

$$
\begin{aligned}
d\left(\boldsymbol{X}_{t}(\boldsymbol{x}) \otimes \boldsymbol{X}_{t}(\boldsymbol{x})\right)=\left(d \boldsymbol{X}_{t}(\boldsymbol{x})\right) \otimes \boldsymbol{X}_{t}(\boldsymbol{x})+\boldsymbol{X}_{t}(\boldsymbol{x}) \otimes\left(d \boldsymbol{X}_{t}(\boldsymbol{x})\right)+\frac{4 k T}{\zeta} \mathrm{Id} d t \\
=\quad\left(-\boldsymbol{u}(t, \boldsymbol{x}) \cdot \nabla\left(\boldsymbol{X}_{t}(\boldsymbol{x}) \otimes \boldsymbol{X}_{t}(\boldsymbol{x})\right)\right. \\
\quad+\nabla \boldsymbol{u}(t, \boldsymbol{x})\left(\boldsymbol{X}_{t}(\boldsymbol{x}) \otimes \boldsymbol{X}_{t}(\boldsymbol{x})\right)+\left(\boldsymbol{X}_{t}(\boldsymbol{x}) \otimes \boldsymbol{X}_{t}(\boldsymbol{x})\right)(\nabla \boldsymbol{u}(t, \boldsymbol{x}))^{T} \\
\left.\quad-\frac{2}{\zeta} \boldsymbol{F}\left(\boldsymbol{X}_{t}\right) \otimes \boldsymbol{X}_{t}-\frac{2}{\zeta} \boldsymbol{X}_{t} \otimes \boldsymbol{F}\left(\boldsymbol{X}_{t}\right)+\frac{4 k T}{\zeta} \mathrm{Id}\right) d t \\
+2 \sqrt{\frac{k T}{\zeta}}\left(\left(\boldsymbol{X}_{t}(\boldsymbol{x}) \otimes d \boldsymbol{W}_{t}\right)+\left(d \boldsymbol{W}_{t} \otimes \boldsymbol{X}_{t}(\boldsymbol{x})\right)\right) .
\end{aligned}
$$

The mean of the structure tensor

$$
\boldsymbol{A}(t, \boldsymbol{x})=\mathbb{E}\left(\boldsymbol{X}_{t}(\boldsymbol{x}) \otimes \boldsymbol{X}_{t}(\boldsymbol{x})\right)
$$


therefore solves, under some mathematical assumptions on $\boldsymbol{X}_{t}$,

$$
\begin{aligned}
& \frac{\partial \boldsymbol{A}}{\partial t}(t, \boldsymbol{x})+\boldsymbol{u}(t, \boldsymbol{x}) \cdot \nabla \boldsymbol{A}(t, \boldsymbol{x})-\nabla \boldsymbol{u}(t, \boldsymbol{x}) \boldsymbol{A}(t, \boldsymbol{x})-\boldsymbol{A}(t, \boldsymbol{x})(\nabla \boldsymbol{u}(t, \boldsymbol{x}))^{T} \\
&=-\frac{4}{\zeta} \mathbb{E}\left(\boldsymbol{X}_{t} \otimes \boldsymbol{F}\left(\boldsymbol{X}_{t}\right)\right)+\frac{4 k T}{\zeta} \mathrm{Id} .
\end{aligned}
$$

Using (4.30), the following expression of the stress tensor, called the Giesekus formula, is obtained, which only explicitly depends on second moments of $\boldsymbol{X}_{t}$ :

$$
\begin{aligned}
& \boldsymbol{\tau}_{p}(t, \boldsymbol{x})= \\
& \quad-\frac{\zeta}{4} n_{p}\left(\frac{\partial \boldsymbol{A}}{\partial t}(t, \boldsymbol{x})+\boldsymbol{u}(t, \boldsymbol{x}) . \nabla \boldsymbol{A}(t, \boldsymbol{x})-\nabla \boldsymbol{u}(t, \boldsymbol{x}) \boldsymbol{A}(t, \boldsymbol{x})-\boldsymbol{A}(t, \boldsymbol{x})(\nabla \boldsymbol{u}(t, \boldsymbol{x}))^{T}\right) .
\end{aligned}
$$

The stress $\boldsymbol{\tau}_{p}$ is thus proportional to the upper-convected derivative of $\boldsymbol{A}$.

\subsubsection{The force}

We now have to make the force $\boldsymbol{F}$ specific. In full generality, it is assumed that $\boldsymbol{F}$ is the gradient of a convex, radially symmetric, potential $\Pi(\boldsymbol{X})=\pi(\|\boldsymbol{X}\|)$. Thus,

$$
\boldsymbol{F}(\boldsymbol{X})=\pi^{\prime}(\|\boldsymbol{X}\|) \frac{\boldsymbol{X}}{\|\boldsymbol{X}\|} .
$$

The convexity of $\Pi(\boldsymbol{X})$ with respect to $\boldsymbol{X}$ of course amounts to that of $\pi(l)$ with respect to $l$, together with $\pi^{\prime}(0) \geq 0$.

The simplest example of potential $\pi$ is the quadratic potential $\pi_{\text {Hook }}(l)=H \frac{l^{2}}{2}$, which of course corresponds to the Hookean force introduced in (3.10). There are two major pitfalls with the Hookean dumbbell model: first it is not a multiscale model in nature, and second (and perhaps more importantly), it has a highly non physical feature.

Let us begin by verifying that the Hookean model is actually equivalent to the purely macroscopic Oldroyd B model introduced in (2.22).

More on the Hookean model For Hookean dumbbell, we have: $\mathbb{E}(\boldsymbol{X} \otimes \boldsymbol{F}(\boldsymbol{X}))=$ $H \mathbb{E}(\boldsymbol{X} \otimes \boldsymbol{X})$, thus the following equation is obtained on the structure tensor $\boldsymbol{A}=\mathbb{E}(\boldsymbol{X} \otimes \boldsymbol{X})$ :

$$
\begin{array}{r}
\frac{\partial \boldsymbol{A}}{\partial t}(t, \boldsymbol{x})+\boldsymbol{u}(t, \boldsymbol{x}) . \nabla \boldsymbol{A}(t, \boldsymbol{x})-\nabla \boldsymbol{u}(t, \boldsymbol{x}) \boldsymbol{A}(t, \boldsymbol{x})-\boldsymbol{A}(t, \boldsymbol{x})(\nabla \boldsymbol{u}(t, \boldsymbol{x}))^{T} \\
=-\frac{4 H}{\zeta} \boldsymbol{A}(t, \boldsymbol{x})+\frac{4 k T}{\zeta} \mathrm{Id},
\end{array}
$$

that is, in terms of $\boldsymbol{\tau}_{p}$ :

$$
\begin{array}{r}
\frac{\zeta}{4 H}\left(\frac{\partial \boldsymbol{\tau}_{p}}{\partial t}(t, \boldsymbol{x})+\boldsymbol{u}(t, \boldsymbol{x}) . \nabla \boldsymbol{\tau}_{p}(t, \boldsymbol{x})-\nabla \boldsymbol{u}(t, \boldsymbol{x}) \boldsymbol{\tau}_{p}(t, \boldsymbol{x})-\boldsymbol{\tau}_{p}(t, \boldsymbol{x})(\nabla \boldsymbol{u}(t, \boldsymbol{x}))^{T}\right) \\
=-\boldsymbol{\tau}_{p}(t, \boldsymbol{x})+n_{p} k T \frac{\zeta}{4 H}\left(\nabla \boldsymbol{u}(t, \boldsymbol{x})+(\nabla \boldsymbol{u}(t, \boldsymbol{x}))^{T}\right) .
\end{array}
$$


Introducing the relaxation time

$$
\lambda=\frac{\zeta}{4 H}
$$

and the viscosity

$$
\eta_{p}=n_{p} k T \lambda,
$$

we recognize the macroscopic Maxwell (or Oldroyd B) model (2.22), that is,

$$
\lambda\left(\frac{\partial \boldsymbol{\tau}_{p}}{\partial t}+\boldsymbol{u} \cdot \nabla \boldsymbol{\tau}_{p}-\nabla \boldsymbol{u} \boldsymbol{\tau}_{p}-\boldsymbol{\tau}_{p}(\nabla \boldsymbol{u})^{T}\right)+\boldsymbol{\tau}_{p}=\eta_{p} \dot{\boldsymbol{\gamma}}
$$

A few other multiscale models have a macroscopic equivalent. This is for example the case of the FENE-P model (see Equation (4.40) below), which is deliberately built to have a macroscopic equivalent. But for most other multiscale models of real interest (in particular those involving FENE forces, see Equation (4.39) below), no macroscopic equivalent formulation is known. And it is believed that no such formulation exists. In this latter sense, multiscale models are more powerful than purely macroscopic models.

In addition to the above, a major theoretical flaw of the dumbbell model, exemplified in (3.10), is that nothing prevents the end-to-end vector, in the Hookean model, to reach arbitrarily large lengths $|\boldsymbol{r}|$. This is of course not consistent with the actual finite length of the chain. This indeed comes from the method of derivation where we have taken the limit of large $N$, each of the link being of length $a$. In the limit, the total length of the chain therefore explodes, thus the formula (3.10). For all the above reasons, the Hookean dumbbell model, although a perfect test case for preliminary mathematical arguments, is not a fully appropriate benchmark, physically, mathematically and numerically representative, for multiscale models.

Accounting for the finite extensibility of the chain is an important issue, for which adequate models exist. We now turn to two of them.

Other forces The FENE model, where FENE is the acronym for Finite Extensible Nonlinear Elastic, is perhaps the most famous force field employed in the simulation of polymeric fluids. It corresponds to the potential (see Figure 10):

$$
\pi_{\mathrm{FENE}}(l)=-\frac{b k T}{2} \ln \left(1-\frac{l^{2}}{b k T / H}\right) .
$$

The success of this potential is well recognized. In this mathematical text, it is not our purpose to argue on the physical validity and relevance of the models. However, an interesting point to make is the following. The dumbbell model is a very coarse model of the polymer chain. Taking two beads to model a thousand-atom chain seems oversimplifying. When equipped with an appropriate entropic force, like the FENE force, this model nevertheless yields tremendously good results. From a general viewpoint, this shows that

- a multiscale model is much more powerful than a purely macroscopic model, 
- the description of the microstructure does not need to be sophisticated to give excellent results,

- it only has to capture the right physics (see the FENE force in contrast to the Hookean force).

Note also that, as a counterpart to the above, the FENE model raises a huge number of challenging mathematical and numerical questions. We will address some of them in Section 6]

The FENE model cannot be rephrased under the form of a purely macroscopic model. There is no proof of this claim, but it is strongly believed to be the case. For some specific purposes, the idea has arisen to find a modification of the FENE model (a so-called closure approximation) which would have a macroscopic equivalent. This gives birth to the FENE-P model, where $P$ stands for Peterlin. Following A. Peterlin [104] and R.B. Bird, P.J. Dotson and N.L. Johnson [13], it has indeed been proposed to replace the denominator of the FENE force (4.39) by a mean value of the elongation:

$$
\boldsymbol{F}_{\mathrm{FENE}-\mathrm{P}}\left(\boldsymbol{X}_{t}\right)=\frac{H \boldsymbol{X}_{t}}{1-\frac{\mathbb{E}\left(\left\|\boldsymbol{X}_{t}\right\|^{2}\right)}{b k T / H}} .
$$

Accordingly, the microscopic description of the fluids now reads:

$$
\left\{\begin{array}{l}
\boldsymbol{\tau}_{p}=n_{p}\left(\frac{H \mathbb{E}\left(\boldsymbol{X}_{t} \otimes \boldsymbol{X}_{t}\right)}{1-\mathbb{E}\left(\left\|\boldsymbol{X}_{t}\right\|^{2}\right) /(b k T / H)}-k T \mathrm{Id}\right), \\
d \boldsymbol{X}_{t}+\boldsymbol{u} \cdot \nabla \boldsymbol{X}_{t} d t=\left(\nabla \boldsymbol{u} \boldsymbol{X}_{t}-\frac{2 H}{\zeta} \frac{\boldsymbol{X}_{t}}{1-\mathbb{E}\left(\left\|\boldsymbol{X}_{t}\right\|^{2}\right) /(b k T / H)}\right) d t+2 \sqrt{\frac{k T}{\zeta}} d \boldsymbol{W}_{t} .
\end{array}\right.
$$

Using the expression of $\tau_{p}$, 4.30) and (4.34), we obtain:

$$
\begin{aligned}
\frac{\partial \boldsymbol{A}}{\partial t}(t, \boldsymbol{x})+\boldsymbol{u}(t, \boldsymbol{x}) . \nabla \boldsymbol{A}(t, \boldsymbol{x}) & -\nabla \boldsymbol{u}(t, \boldsymbol{x}) \boldsymbol{A}(t, \boldsymbol{x})-\boldsymbol{A}(t, \boldsymbol{x})(\nabla \boldsymbol{u}(t, \boldsymbol{x}))^{T} \\
& =-\frac{4 H}{\zeta} \frac{\boldsymbol{A}(t)}{1-\operatorname{tr}(\boldsymbol{A}(t)) /(b k t / H)}+\frac{4 k T}{\zeta} \mathrm{Id} .
\end{aligned}
$$

Inserting this into:

$$
\boldsymbol{A}=\frac{1}{H Z\left(\operatorname{tr}\left(\boldsymbol{\tau}_{p}\right)\right)}\left(\frac{\boldsymbol{\tau}_{p}}{n_{p}}+k T \mathrm{Id}\right),
$$

where $Z$ is defined by (2.29), the following equation is obtained on $\tau_{p}$ :

$$
\begin{array}{r}
\frac{\zeta}{4 H}\left(\frac{\partial \boldsymbol{\tau}_{p}}{\partial t}(t, \boldsymbol{x})+\boldsymbol{u}(t, \boldsymbol{x}) . \nabla \boldsymbol{\tau}_{p}(t, \boldsymbol{x})-\nabla \boldsymbol{u}(t, \boldsymbol{x}) \boldsymbol{\tau}_{p}(t, \boldsymbol{x})-\boldsymbol{\tau}_{p}(t, \boldsymbol{x})(\nabla \boldsymbol{u}(t, \boldsymbol{x}))^{T}\right) \\
+Z\left(\operatorname{tr}\left(\boldsymbol{\tau}_{p}\right)\right) \boldsymbol{\tau}_{p}-\frac{\zeta}{4 H}\left(\boldsymbol{\tau}_{p}+n_{p} k T \operatorname{Id}\right)\left(\frac{\partial}{\partial t}+\boldsymbol{u} \cdot \nabla\right) \ln \left(Z\left(\operatorname{tr}\left(\boldsymbol{\tau}_{p}\right)\right)\right) \\
=n_{p} k T \frac{\zeta}{4 H}\left(\nabla \boldsymbol{u}(t, \boldsymbol{x})+(\nabla \boldsymbol{u}(t, \boldsymbol{x}))^{T}\right)
\end{array}
$$




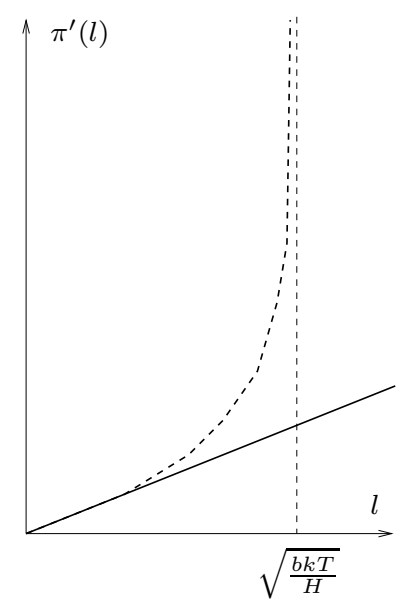

Figure 10: Comparison of the Hookean force (continuous line) and the FENE force (dashed line).

which is exactly the FENE-P model mentioned in 2.28 (when $\lambda$ and $\eta_{p}$ are respectively given by (4.37) and (4.38). The FENE-P model can thus be seen as a modification of the FENE model, in order to obtain a multiscale model that has an equivalent purely macroscopic formulation. Other variants of the FENE model exist in the literature.

\subsection{The multiscale model}

We now have all the bricks for the stochastic variant of our multiscale system (3.13). Collecting the material of the previous section, we obtain:

$$
\left\{\begin{array}{l}
\rho\left(\frac{\partial \boldsymbol{u}}{\partial t}(t, \boldsymbol{x})+\boldsymbol{u}(t, \boldsymbol{x}) \cdot \nabla \boldsymbol{u}(t, \boldsymbol{x})\right)-\eta \Delta \boldsymbol{u}(t, \boldsymbol{x})+\nabla p(t, \boldsymbol{x}) \\
\quad=\operatorname{div}\left(\boldsymbol{\tau}_{p}(t, \boldsymbol{x})\right)+\rho \boldsymbol{f}(t, \boldsymbol{x}), \\
\operatorname{div}(\boldsymbol{u}(t, \boldsymbol{x}))=0, \\
\boldsymbol{\tau}_{p}(t, \boldsymbol{x})=n_{p}\left(\mathbb{E}\left(\boldsymbol{X}_{t}(\boldsymbol{x}) \otimes \boldsymbol{F}\left(\boldsymbol{X}_{t}(\boldsymbol{x})\right)\right)-k T \mathrm{Id}\right), \\
d \boldsymbol{X}_{t}(\boldsymbol{x})+\boldsymbol{u}(t, \boldsymbol{x}) \cdot \nabla \boldsymbol{X}_{t}(\boldsymbol{x}) d t \\
\quad=\nabla \boldsymbol{u}(t, \boldsymbol{x}) \boldsymbol{X}_{t}(\boldsymbol{x}) d t-\frac{2}{\zeta} \boldsymbol{F}\left(\boldsymbol{X}_{t}(\boldsymbol{x})\right) d t+2 \sqrt{\frac{k T}{\zeta}} d \boldsymbol{W}_{t} .
\end{array}\right.
$$


As was the case for the Fokker-Planck equation, the stochastic differential equations are to be solved at each point of the macroscopic flow. The process $\boldsymbol{X}_{t}$ therefore implicitly depends on $\boldsymbol{x}$.

It is well-known that the form of equations actually used in the numerical practice is a non-dimensional form. Because this involves the introduction of several non-dimensional numbers that have a physical meaning and are present in the literature, let us briefly establish now this non-dimensional form for (4.44) (and thus for (3.13), by analogy, see (3.14)).

We introduce the following characteristic quantities: $U$ the characteristic velocity, $L$ the characteristic length, $\lambda=\frac{\zeta}{4 H}$, as in (4.37), the characteristic relaxation time, $\eta_{p}=n_{p} k T \lambda$, as in (4.38), the viscosity of polymers. Then, we consider the following non-dimensional numbers:

$$
\begin{cases}\mathrm{Re}=\frac{\rho U L}{\eta}, & \epsilon=\frac{\eta_{p}}{\eta}, \\ \mathrm{We}=\frac{\lambda U}{L}, & \mu=\frac{L^{2} H}{k T} .\end{cases}
$$

respectively the Reynolds number Re measuring the ratio of inertia over viscosity (usually for the complex fluids under consideration, Re $\leq 10), \epsilon$ measuring the ratio of viscosity of the polymers over the total viscosity (usually $\epsilon \approx 0.1$ ), We the Weissenberg number (also called Deborah number) which is the ratio of the relaxation time of the polymers versus the characteristic time of the flow (usually $0.1 \leq \mathrm{We} \leq 10$ ), and $\mu$ measuring a ratio of lengths.

Adimensionalizing also the force by $\boldsymbol{F}\left(\overline{\boldsymbol{X}_{t}}\right)=\frac{\boldsymbol{F}\left(L \overline{\boldsymbol{X}_{t}}\right)}{H L}$, and taking (which is the commonly used value) $\mu=1$, we obtain:

$$
\left\{\begin{array}{l}
\operatorname{Re}\left(\frac{\partial \boldsymbol{u}}{\partial_{t}}+\boldsymbol{u} \cdot \nabla \boldsymbol{u}\right)-(1-\epsilon) \Delta \boldsymbol{u}+\nabla p=\operatorname{div} \boldsymbol{\tau}_{p}+\boldsymbol{f} \\
\operatorname{div} \boldsymbol{u}=0 \\
\boldsymbol{\tau}_{p}=\frac{\epsilon}{\mathrm{We}}\left(\mathbb{E}\left(\boldsymbol{X}_{t} \otimes \boldsymbol{F}\left(\boldsymbol{X}_{t}\right)\right)-\mathrm{Id}\right) \\
d \boldsymbol{X}_{t}+\boldsymbol{u} \cdot \nabla \boldsymbol{X}_{t} d t=\nabla \boldsymbol{u} \boldsymbol{X}_{t} d t-\frac{1}{2 \mathrm{We}} \boldsymbol{F}\left(\boldsymbol{X}_{t}\right) d t+\frac{1}{\sqrt{\mathrm{We}}} d \boldsymbol{W}_{t} .
\end{array}\right.
$$

An important practical remark stems from the actual range of parameters mentioned above. In contrast to the usual setting of computational fluid mechanics where the challenge is to deal with flows with high Reynolds numbers, the challenge here is not the Reynolds number (kept relatively small), but the Weissenberg number. Tremendous practical (and also, actually, theoretical) difficulties are associated with the so-called High Weissenberg number problem ("high" meaning exceeding, say, 10). 


\subsection{Schematic overview of the simulation}

Our focus so far has been the modelling difficulties for viscoelastic fluids. Another question is the discretization of the models, and their numerical simulations. This has to be performed very carefully since a model is typically validated by some comparisons between experiments and numerical simulations on simple or complex flows.

The present section summarizes the issues and techniques, in a language accessible to readers familiar with scientific computing and numerical analysis. A much more elementary presentation will be given in Section 5

\subsubsection{Numerical methods}

Most of the numerical methods are based upon a finite element discretization in space and Euler schemes in time, using a semi-explicit scheme: at each timestep, the velocity is first solved for a given stress, and then the stress is updated, for a fixed velocity.

In the case of micro-macro models such as (3.14) and (4.46), another discretization step is necessary to approximate the expectation or the integral in the definition of the stress tensor $\tau_{p}$. There are basically two methods of discretization, depending on the formulation used: stochastic methods for (4.46), and deterministic methods for (3.14).

To discretize the expectation in (4.46), a Monte Carlo method is employed: at each macroscopic point $\boldsymbol{x}$ (i.e. at each node of the mesh once the problem is discretized) many replicas (or realizations) $\left(\boldsymbol{X}_{t}^{k, K}\right)_{1 \leq k \leq K}$ of the stochastic process $\boldsymbol{X}_{t}$ are simulated, driven by independent Brownian motions $\left(\boldsymbol{W}_{t}^{\bar{k}}\right)_{k \geq 1}$, and the stress tensor is obtained as an empirical mean over these processes:

$$
\boldsymbol{\tau}_{p}^{K}=\frac{\epsilon}{\mathrm{We}}\left(\frac{1}{K} \sum_{k=1}^{K} \boldsymbol{X}_{t}^{k, K} \otimes \boldsymbol{F}\left(\boldsymbol{X}_{t}^{k, K}\right)-\mathrm{Id}\right)
$$

In this context, this discretization method coupling a finite element method and a Monte Carlo technique is called CONNFFESSIT for Calculation Of Non-Newtonian Flow: Finite Elements and Stochastic SImulation Technique (see M. Laso and H.C. Öttinger [75]). In Section 5, we will implement this method in a simple geometry. Let us already mention that one important feature of the discretization is that, at the discrete level, all the unknowns $\left(\boldsymbol{u}, p, \boldsymbol{\tau}_{p}\right)$ become random variables. The consequence is that the variance of the results is typically the bottleneck for the accuracy of the method. In particular, variance reduction methods are very important.

To discretize the Fokker Planck equation in (3.14), spectral methods are typically used (see A. Lozinski [91] or J.K.C. Suen, Y.L. Joo and R.C. Armstrong [117]). It is not easy to find a suitable variational formulation of the Fokker Planck equation, and an appropriate discretization that satisfies the natural constraints on the probability density $\psi$ (namely non negativity, and normalization). We refer to $\mathrm{C}$. Chauvière and A. Lozinski [25, 92 , for appropriate discretization in the FENE case. One major difficulty in the discretization of Fokker-Planck equations is when the configurational space is high-dimensional. In the 
context of polymeric fluid flow simulation, when the polymer chain is modelled by a chain of $N$ beads linked by springs, the Fokker-Planck equation is a parabolic equation posed on a $3 \mathrm{~N}$-dimensional domain. Some numerical methods have been developed to discretize such high dimensional problems. The idea is to use an appropriate Galerkin basis, whose dimension does not explode when dimension grows. We refer to P. Delaunay, A. Lozinski and R.G. Owens [33], T. von Petersdorff and C. Schwab [119, H.-J. Bungartz and M. Griebel [20] for the sparse-tensor product approach, to L. Machiels, Y. Maday, and A.T. Patera [93] for the reduced basis approach and to A. Ammar, B. Mokdad, F. Chinesta and R. Keunings [2, 3 for a method coupling a sparse-tensor product discretization with a reduced approximation basis approach.

\subsubsection{Main difficulties}

It actually turns out that the discretization of micro-macro models such as (3.14) and (4.46) or that of macro-macro models such as (2.23) is not trivial. Let us mention three kinds of difficulties:

1. Some inf-sup condition must be satisfied by the spaces respectively used for the discrete velocity, pressure and stress (if one wants the discretization to be stable for $\epsilon$ close to 1$)$.

2. The advection terms need to be discretized correctly, in the conservation of momentum equations, in the equation on $\boldsymbol{\tau}_{p}$ in (2.23), in the equation on $\psi$ in (3.14), on in the $\mathrm{SDE}$ in 4.46).

3. The nonlinear terms require, as always, a special care. On the one hand, some nonlinear terms stem from the coupling: $\nabla \boldsymbol{u} \boldsymbol{\tau}_{p}+\boldsymbol{\tau}_{p}(\nabla \boldsymbol{u})^{T}$ in (2.23), $\nabla \boldsymbol{u} \boldsymbol{X}_{t}$ in (4.46) or $\operatorname{div} \boldsymbol{X}_{\boldsymbol{X}}(\nabla \boldsymbol{u} \boldsymbol{X} \psi(t, \boldsymbol{x}, \boldsymbol{X}))$ in (3.14). On the other hand, for rheological models more complicated than Oldroyd-B or Hookean dumbbell, some nonlinear terms come from the model itself (see the entropic force $\boldsymbol{F}\left(\boldsymbol{X}_{t}\right)$ in (4.46) for FENE model for example).

Besides, for both micro-macro models and purely macroscopic models, one central difficulty of the simulation of viscoelastic fluids is the so-called High Weissenberg Number Problem (HWNP). It is indeed observed that numerical simulations do not converge when We is too large. The maximum value which can be actually correctly simulated depends on the geometry of the problem (4:1 contraction, flow past a cylinder,...), on the model (Oldroyd-B model, FENE model, ...) and also on the discretization method. Typically, it is observed that this maximum value decreases with mesh refinement.

We will return to these questions in Section [6] 


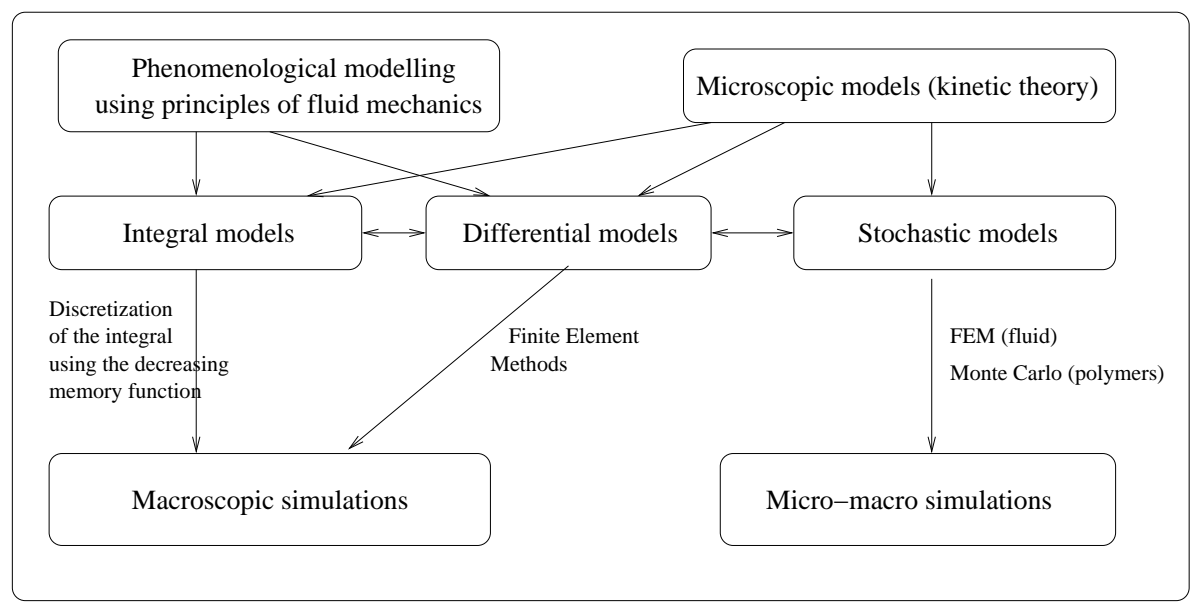

Figure 11: Macro-macro and micro-macro models for complex fluids.

\subsection{Upsides and downsides of multiscale modelling for complex flu- ids}

\subsubsection{Micro-macro vs macro-macro modelling}

We are now in position to compare the micro-macro approach and the macro-macro approach to simulate polymeric fluids (and more generally complex fluids). Figure[1] summarizes the main features of these approaches. Let us discuss this from two viewpoints: modelling and numerics.

From the modelling viewpoint, the interest of the micro-macro approach stems from the fact it is based on a clear understanding of the physics at play. The kinetic equations used to model the evolution of the polymers are well established and the limit of the validity of these equations is known. The constants involved in micro-macro models have a clear physical signification, and can be estimated from some microscopic properties of the polymer chains. From this point of view, the micro-macro approach seems more predictive, and enables an exploration of the link between the microscopic properties of the polymer chains (or more generally the microstructures in the fluid) and the macroscopic behavior of the complex fluid.

Practice confirms this. It indeed appears that simulations with micro-macro models generally compare better to experiments (see R. Keunings [71, 72]). However, for complex flows and general non-Newtonian fluids, it is still difficult to agree quantitatively with the experiments. In short, it remains a lot to do from the modelling viewpoint, but it is generally admitted that the micro-macro approach is the most promising way to improve the models. 
From the numerical point of view, the major drawback of the micro-macro approach is its computational cost. The introduction of an additional field to describe the configuration of the microstructure in the fluid implies additional computations and additional memory storage.

For example, for the micro-macro models introduced above in their stochastic form (4.46), the discretization by a CONNFFESSIT approach requires the storage at each node of the mesh of an ensemble of configurations $\left(\boldsymbol{X}_{t}^{i, M}\right)_{1 \leq i \leq M}$ of the polymer chains. Even though the SDEs associated to each configuration, and at various node of the mesh can be solved in parallel on each time step, the computational cost remains very high. The micro-macro approach is currently not sufficiently efficient to be used in commercial codes for industrial purposes.

In view of the arguments above, it seems natural to try and design some numerical methods that couple the macro-macro and the micro-macro approaches. The macro-macro model is used where the flow is simple, and the detailed micro-macro model is used elsewhere. The idea of adaptive modelling based on modelling error a posteriori analysis (see J.T. Oden and K.S. Vemaganti 99, J.T. Oden and S. Prudhomme 98 or M. Braack and A. Ern [19] has been recently adapted in this context in a preliminary work by A. Ern and T. Lelièvre [40].

We mentioned above the problems raised by the discretization of macro-macro and micromacro models. It seems that in complex flows, numerical methods based on the micro-macro approach are more robust than those based on the macro-macro approach (see A.P.G. Van Heel [118, p.38], J.C. Bonvin [18, p.115] or C. Chauvière [24]). However, this is not yet well understood mathematically. In addition, the HWNP still limits the range of applicability of the computations, even with micro-macro models. The main interest of micro-macro approaches as compared to macro-macro approaches lies at the modelling level. It may become the method of choice for a backroom strategy. The approach allows to test and validate purely macroscopic models, to supply such models with adequate and reliable boundary conditions, etc..., even if, in the state of the art technology, it does not allow to perform simulations for actual real-world applications, owing to its extremely computationally demanding nature.

\subsubsection{Fokker-Planck $v s$ SDE formulation}

To conclude this section, we would like to discuss the advantages and drawbacks of the two numerical approaches introduced above for the micro-macro approach: that based on the deterministic formulation (3.14) and that based on the stochastic formulation (4.46).

The conclusions of this comparison (see Section [5] and also A. Lozinski and C. Chauvière [92]) are actually very general: when it is possible to use the deterministic approach (discretization of the Fokker-Planck PDE), it is much more efficient than the stochastic approach (Monte Carlo methods to approximate the expectation). The main reason for that is that the convergence of a Monte Carlo method is slower than that of a deterministic approximation method.

The following question is then: what are the limits of the Fokker-Planck approach? As we mentioned above, designing a numerical method that satisfies the natural requirements 


\begin{tabular}{c|c|c|c}
\cline { 2 - 4 } & MACRO & \multicolumn{2}{|c}{ MICRO-MACRO } \\
\hline modelling capabilities & low & \multicolumn{2}{|c}{ high } \\
\hline current utilization & industry & \multicolumn{2}{|c}{ laboratories } \\
\hline computational cost & low & hiscretization & $\begin{array}{c}\text { discretization } \\
\text { of Fokker-Planck }\end{array}$ \\
\hline computational bottleneck & HWNP & variance, HWNP & dimension, HWNP \\
\hline
\end{tabular}

Table 1: Summary of the characteristics of macro-macro and micro-macro approaches for the simulation of complex fluids.

of non-negativity and normalization of $\psi$ is not an easy task. In the FENE case, proper variational formulations are to be employed, which take into account the boundary conditions on $\psi$. In practice, it is observed that the stability of numerical schemes deteriorates when $\nabla \boldsymbol{u}$ becomes too large. But there is another (more fundamental) limitation to the deterministic approach. We mentioned above that the dumbbell model may be actually too crude to describe correctly the polymer chain configuration in some specific situations. It might be better, then, to use a chain of beads and springs. For such a model, the stochastic approach and the associated discretization can both be generalized straightforwardly. However, the deterministic approach is much more problematic. The Fokker-Planck equation becomes a high-dimensional PDE, and the discretization is very difficult. We mentioned above some numerical methods to deal with such PDEs (the sparse-tensor product approach, the reduced approximation basis approach) but they are still limited to a relatively small number of springs, and are much more difficult to implement than Monte Carlo methods.

A summary of the comparison of the various approaches to model complex fluids is given in Table 1 


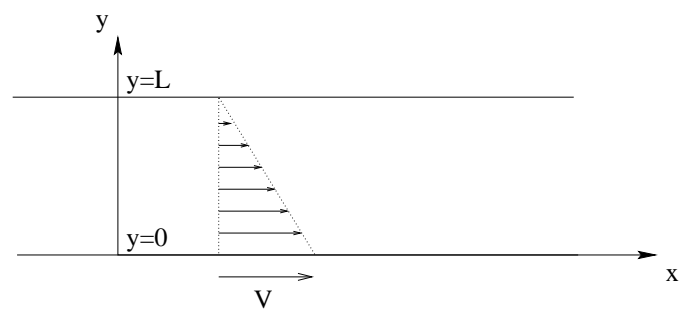

Figure 12: Couette flow.

\section{Numerical simulation of a test case: the Couette flow}

\subsection{Setting of the problem}

We consider in this section the simple situation of a start-up Couette flow (see Figure 12). The fluid flows between two parallel planes. Such a model is typically obtained considering a flow in a rheometer, between two cylinders, and taking the limit of large radii for both the inner and the outer cylinders (see Figure 1). At initial time, the fluid is at rest. The lower plane ( $y=0$, modelling the inner cylinder of the rheometer) is then shifted with a velocity $V(t)$, which, for simplicity, will be set to a constant value $V$ (sinusoidal velocities may also be applied):

$$
V(t)=V .
$$

On the other hand, the upper plane ( $y=L$, modelling the outer cylinder of the rheometer) is kept fixed. Such a setting is called a start-up flow, and because it is confined between two parallel plane, a Couette flow.

We denote by $x$ and $y$ the horizontal and vertical axes, respectively. The flow is assumed invariant in the direction perpendicular to $(x, y)$.

The polymeric fluid filling in the space between the planes obeys equations (2.13), which we reproduce here for convenience in their nondimensional form:

$$
\left\{\begin{aligned}
\operatorname{Re}\left(\frac{\partial \boldsymbol{u}}{\partial t}+(\boldsymbol{u} \cdot \nabla) \boldsymbol{u}\right)-(1-\epsilon) \Delta \boldsymbol{u}+\nabla p-\operatorname{div} \boldsymbol{\tau}_{p} & =\boldsymbol{f} \\
\operatorname{div} \boldsymbol{u} & =0
\end{aligned}\right.
$$

For Couette flow, we have $f=0$.

It is natural to assume that the flow is laminar, that is, the velocity writes $\boldsymbol{u}=u_{x}(t, x, y) \boldsymbol{e}_{x}$, where $\boldsymbol{e}_{x}$ is the unitary vector along the $x$-axis. The incompressibility constraint (2.8) immediately implies that $\boldsymbol{u}=u_{x}(t, y) \boldsymbol{e}_{x}$. We now denote:

$$
\boldsymbol{u}=u(t, y) \boldsymbol{e}_{x} .
$$


In the Newtonian case $\left(\tau_{p}=0\right)$, it can be easily shown that a natural assumption on the pressure leads to

$$
\left\{\begin{array}{l}
\operatorname{Re} \frac{\partial u}{\partial t}(t, y)=(1-\epsilon) \frac{\partial^{2} u}{\partial y^{2}}(t, y), \\
u(0, y)=0 \\
u(t, 0)=V \\
u(t, L)=0
\end{array}\right.
$$

Let us now consider the case of a non-Newtonian fluid modelled but the Hookean dumbbell model. We will treat this model as a multiscale model, even if we know from Section 4.2.3 that it is equivalent to the purely macroscopic Oldroyd-B model. Our purpose is indeed to illustrate the numerical approach for such multiscale models, and the Hookean dumbbell model is a nice setting for the exposition. For other models, the situation is more intricate, but at least all the difficulties of the Hookean dumbbell model are present.

In full generality, the Fokker-Planck version of the multiscale system describing the flow for the Hookean dumbbell model reads (again in a non-dimensional form), we recall:

$$
\left\{\begin{array}{l}
\operatorname{Re}\left(\frac{\partial \boldsymbol{u}}{\partial t}+(\boldsymbol{u} \cdot \nabla) \boldsymbol{u}\right)-(1-\epsilon) \Delta \boldsymbol{u}+\nabla p-\operatorname{div} \boldsymbol{\tau}_{p}=0, \\
\operatorname{div} \boldsymbol{u}=0, \\
\boldsymbol{\tau}_{p}(t, x, y)=\frac{\epsilon}{\mathrm{We}}\left(\int(\boldsymbol{r} \otimes \boldsymbol{r}) \psi(t, x, y, \boldsymbol{r}) d \boldsymbol{r}-\mathrm{Id}\right), \\
\frac{\partial \psi}{\partial t}(t, x, y, \boldsymbol{r})+\boldsymbol{u}(t, x, y) \cdot \nabla_{x, y} \psi(t, x, y, \boldsymbol{r}) \\
\quad=-\operatorname{div}_{\boldsymbol{r}}\left(\left(\nabla_{x, y} \boldsymbol{u}(t, x, y) \boldsymbol{r}-\frac{1}{2 \mathrm{We}} \boldsymbol{r}\right) \psi(t, x, y, \boldsymbol{r})\right)+\frac{1}{2 \mathrm{We}} \Delta_{\boldsymbol{r}} \psi(t, x, y, \boldsymbol{r}) .
\end{array}\right.
$$

supplied with

$$
\begin{cases}\boldsymbol{u}(0, x, y)=\mathbf{0}, & \\ \boldsymbol{u}(t, x, y=0)=V \boldsymbol{e}_{x}, & \forall t>0, \\ \boldsymbol{u}(t, x, y=L)=\overrightarrow{0}, & \forall t>0\end{cases}
$$

Owing to the specific Couette setting, and the assumptions that originate from it (notably (5.2)), the above general system simplifies into the much simpler one:

$$
\left\{\begin{array}{l}
\operatorname{Re} \frac{\partial u}{\partial t}(t, y)=(1-\epsilon) \frac{\partial^{2} u}{\partial y^{2}}(t, y)+\frac{\partial \tau}{\partial y}(t, y), \\
\tau(t, y)=\frac{\epsilon}{\mathrm{We}} \int_{\mathbb{R}^{2}} P Q \psi(t, y, P, Q) d P d Q, \\
\frac{\partial \psi}{\partial t}(t, y, P, Q)=-\frac{\partial}{\partial P}\left(\left(\frac{\partial u}{\partial y}(t, y) Q-\frac{1}{2 \mathrm{We}} P\right) \psi(t, y, P, Q)\right) \\
\quad+\frac{\partial}{\partial Q}\left(\frac{1}{2 \mathrm{We}} Q \psi(t, y, P, Q)\right)+\frac{1}{2 \mathrm{We}}\left(\frac{\partial^{2}}{\partial P^{2}}+\frac{\partial^{2}}{\partial Q^{2}}\right) \psi(t, y, P, Q),
\end{array}\right.
$$


where $P$ and $Q$ are the two components of the end-to-end vector $\boldsymbol{r}$, along the $x$ and $y$ axes respectively. In the above system, $\tau(t, y)$ denotes the $x y$ entry of the tensor $\boldsymbol{\tau}_{p}$. Actually, the pressure field, and the other entries of the stress tensor may be then deduced, independently.

Let us emphasize at this stage the tremendous simplifications that the Couette model allows for. Owing to the simple geometric setting and the fact that the flow is assumed laminar, the divergence-free constraint (2.8) is fulfilled by construction of the velocity field and can be eliminated from the system. In addition, the transport terms $(\boldsymbol{u} \cdot \nabla) \boldsymbol{u}$ and $(\boldsymbol{u} \cdot \nabla) \psi$ cancel out, again because of geometrical considerations. This explains the extremely simple form of the equation of conservation of momentum in this context, which indeed reduces to a simple one-dimensional heat equation. This set of simplifications is specific to the Couette flow. Substantial difficulties arise otherwise.

We now describe the numerical approach for (5.6). To begin with, we present the (simple) finite element discretization of the macroscopic equation. Then we turn to the numerical approach employed for the Fokker-Planck equation. The variant using a stochastic differential equation then follows.

\subsection{Discretization of the macroscopic equation}

Let us consider the stress $\tau(t, y)$ is known, and perform the variational formulation of the equation in (5.6) determining the velocity

$$
\operatorname{Re} \frac{\partial u}{\partial t}(t, y)=(1-\epsilon) \frac{\partial^{2} u}{\partial y^{2}}(t, y)+\frac{\partial \tau}{\partial y}(t, y)
$$

with a view, next, to discretize it using finite elements. Our formulation is

$$
\left\{\begin{array}{l}
\text { Search for } u:[0, T] \longrightarrow H_{1}^{1}(0, L) \text { such that } \\
\forall v \in H_{0}^{1}(0, L), \quad \operatorname{Re} \frac{d}{d t}(u(t), v)_{L^{2}}=-(1-\epsilon)\left(\frac{\partial u}{\partial y}(t), \frac{\partial v}{\partial y}\right)_{L^{2}}-\left(\tau(t), \frac{\partial v}{\partial y}\right)_{L^{2}}, \\
u(0, y)=0,
\end{array}\right.
$$

where we have denoted

$$
H_{0}^{1}(0, L)=\left\{v \in H^{1}(0, L), \quad v(0)=0, \quad v(L)=0\right\}
$$

and

$$
H_{1}^{1}(0, L)=\left\{v \in H^{1}(0, L), \quad v(0)=1, \quad v(L)=0\right\} .
$$

As regards the discretization, we introduce the shape functions for P1 finite elements (for the velocity)

$$
\varphi_{i}(y)= \begin{cases}1 & \text { when } y=\frac{i}{N} \\ \text { affine } & \text { on }\left[\frac{i-1}{N}, \frac{i}{N}\right] \text { and }\left[\frac{i}{N}, \frac{i+1}{N}\right] \\ 0 & \text { when } y \in\left[0, \frac{i-1}{N}\right] \cup\left[\frac{i+1}{N}, 1\right]\end{cases}
$$


(for $0 \leq i \leq N$ ), with the obvious adaptations when $i=0$ and $i=N$, and the shape functions for P0 finite elements (for the stress)

$$
\chi_{i}(y)= \begin{cases}1 & \text { when } y \in\left[\frac{i-1}{N}, \frac{i}{N}\right), \\ 0 & \text { otherwise }\end{cases}
$$

(for $1 \leq i \leq N$ ), both on a regular mesh over $[0, L]$, with meshsize $h=\Delta y=\frac{1}{N}$. The approximations for $\tau$ and $u$ then read

$$
\begin{gathered}
\tau^{h}(t, y)=\sum_{i=1}^{N}\left(\tau^{h}\right)_{i}(t) \chi_{i}(y), \\
u^{h}(t, y)=\sum_{i=1}^{N-1}\left(u^{h}\right)_{i}(t) \varphi_{i}(y)+V \varphi_{N}(y),
\end{gathered}
$$

respectively. Note indeed, that, because of the boundary condition, we have for all $t>0$, $\left(u^{h}\right)_{0}(t)=0$ and $\left(u^{h}\right)_{N}(t)=V$.

It remains to discretize in time, which we do using a backward Euler scheme for the viscous term. The fully discrete formulation is thus

$$
\left\{\begin{array}{l}
\text { Solve for }\left(u^{h}\right)_{j}^{n} \text { for } j=1, \ldots N-1 \text { and for } n \geq 0 \\
\text { such that }\left(u^{h}\right)_{j}^{0} \equiv 0 \text { and } \forall i=1, \ldots N-1 \\
\operatorname{Re}\left(\frac{\sum_{j=1}^{N-1}\left(u^{h}\right)_{j}^{n+1} \varphi_{j}-\sum_{j=1}^{N-1}\left(u^{h}\right)_{j}^{n} \varphi_{j}}{\Delta t}, \varphi_{i}\right. \\
\quad=(1-\epsilon)\left(\frac{\partial}{\partial y}\left(\sum_{j=1}^{N-1}\left(u^{h}\right)_{j}^{n+1} \varphi_{j}+V \varphi_{N}\right), \frac{\partial}{\partial y} \varphi_{i}\right)_{L^{2}}-\left(\left(\tau^{h}\right)^{n}, \frac{\partial}{\partial y} \varphi_{i}\right)_{L^{2}}
\end{array}\right.
$$

where $\left(\tau^{h}\right)^{n}$ denotes the approximation of $\tau^{h}$ at time $t^{n}$.

In algebraic terms, this writes

$$
\operatorname{Re} M \frac{U^{n+1}-U^{n}}{\Delta t}=-(1-\epsilon) A U^{n+1}-G S^{n}+B
$$

where

$$
U^{n}=\left[\left(u^{h}\right)_{1}^{n}, \ldots,\left(u^{h}\right)_{N-1}^{n}\right]^{T}
$$

is the unknown,

$$
S^{n}=\left[\left(\tau^{h}\right)_{1}^{n}, \ldots,\left(\tau^{h}\right)_{N}^{n}\right]^{T}
$$


and $G$ is a matrix with $(i, j)$-entry

$$
G_{i, j}=\int_{0}^{L} \frac{\partial \varphi_{i}}{\partial y} \chi_{j} d y
$$

The vector $B=-(1-\epsilon) V\left[0, \ldots, 0, \int_{0}^{L} \frac{\partial \varphi_{N}}{\partial y} \frac{\partial \varphi_{N-1}}{\partial y} d y\right]^{T}$ is associated with the Dirichlet boundary condition. The matrices $M$ and $A$ respectively denote the matrices of mass and rigidity of the $\mathrm{P} 1$ finite elements:

$$
\begin{gathered}
M_{i, j}=\int_{0}^{L} \varphi_{i} \varphi_{j} d y \\
A_{i, j}=\int_{0}^{L} \frac{\partial \varphi_{i}}{\partial y} \frac{\partial \varphi_{j}}{\partial y} d y .
\end{gathered}
$$

\subsection{Microscopic problem: the deterministic approach}

We now turn to the discretization of the Fokker-Planck equation in (5.6), that is

$$
\begin{aligned}
\frac{\partial \psi}{\partial t}(t, y, P, Q)= & -\frac{\partial}{\partial P}\left(\left(\frac{\partial u}{\partial y}(t, y) Q-\frac{1}{2 \mathrm{We}} P\right) \psi(t, y, P, Q)\right) \\
& +\frac{\partial}{\partial Q}\left(\frac{1}{2 \mathrm{We}} Q \psi(t, y, P, Q)\right)+\frac{1}{2 \mathrm{We}}\left(\frac{\partial^{2}}{\partial P^{2}}+\frac{\partial^{2}}{\partial Q^{2}}\right) \psi(t, y, P, Q) .
\end{aligned}
$$

Since $y$ is only a parameter, we omit to mention the explicit dependence of $\psi$ upon this parameter throughout this paragraph.

We introduce the equilibrium solution of (5.16) (i.e. the steady state solution of (5.16) for $u=0$ ), namely

$$
\psi_{\infty}(P, Q)=\frac{1}{2 \pi} \exp \left(-\frac{P^{2}+Q^{2}}{2}\right) .
$$

We next change the unknown function in (5.16) setting

$$
\varphi=\frac{\psi}{\psi_{\infty}}
$$

and rewrite (5.16) as

$$
\begin{aligned}
\psi_{\infty} \frac{\partial \varphi}{\partial t}(t, P, Q)= & -\frac{\partial}{\partial P}\left(\frac{\partial u}{\partial y} Q \psi_{\infty} \varphi\right) \\
& +\frac{1}{2 \mathrm{We}} \frac{\partial}{\partial P}\left(\psi_{\infty} \frac{\partial}{\partial P} \varphi\right)+\frac{1}{2 \mathrm{We}} \frac{\partial}{\partial Q}\left(\psi_{\infty} \frac{\partial}{\partial Q} \varphi\right)
\end{aligned}
$$


which is readily semi-discretized in time as

$$
\begin{aligned}
\psi_{\infty} \frac{\varphi_{n+1}-\varphi_{n}}{\Delta t}= & -\frac{\partial}{\partial P}\left(\frac{\partial u}{\partial y} Q \psi_{\infty} \varphi_{n}\right) \\
& +\frac{1}{2 \mathrm{We}} \frac{\partial}{\partial P}\left(\psi_{\infty} \frac{\partial}{\partial P} \varphi_{n+1}\right)+\frac{1}{2 \mathrm{We}} \frac{\partial}{\partial Q}\left(\psi_{\infty} \frac{\partial}{\partial Q} \varphi_{n+1}\right) .
\end{aligned}
$$

A variational formulation of (5.20) on an appropriate functional space $\mathcal{V}$ (see for example B. Jourdain, C. Le Bris, T. Lelièvre and F. Otto [65, Appendix B]) is then:

$$
\left\{\begin{aligned}
\text { Solve for } \varphi_{n} \in \mathcal{V} \text { for } n \geq 0 \text { such that } \forall \theta \in \mathcal{V} \\
\int \frac{\varphi_{n+1}-\varphi_{n}}{\Delta t} \theta \psi_{\infty}=\int \frac{\partial u}{\partial y} Q \frac{\partial \theta}{\partial P} \varphi_{n} \psi_{\infty} \\
-\frac{1}{2 \mathrm{We}} \int \frac{\partial \theta}{\partial P} \frac{\partial \varphi_{n+1}}{\partial P} \psi_{\infty}-\frac{1}{2 \mathrm{We}} \int \frac{\partial \theta}{\partial Q} \frac{\partial \varphi_{n+1}}{\partial Q} \psi_{\infty} \\
\varphi_{0}=1 .
\end{aligned}\right.
$$

The most appropriate basis to use for the Galerkin basis in (5.21) is a basis consisting of (tensor products of) Hermite polynomials $H_{i}$ :

$$
\chi_{i, j}(P, Q)=H_{i}(P) H_{j}(Q),
$$

where

$$
H_{0}(P)=1, \quad H_{1}(P)=P, \quad H_{2}(P)=\frac{1}{\sqrt{2}}\left(P^{2}-1\right)
$$

Indeed, since

$$
\frac{1}{\sqrt{2 \pi}} \int_{\mathbb{R}} H_{i}(P) H_{j}(P) \exp \left(-P^{2} / 2\right) d P=\delta_{i j},
$$

and since the Gaussian function is precisely the stationary solution to the equation under consideration, the basis of Hermite polynomials is well adapted to the problem under consideration. In particular, the mass matrix related to the discretization of $\int \frac{\varphi_{n+1}-\varphi_{n}}{\Delta t} \theta \psi_{\infty}$ in (5.21) is the identity matrix. The matrix associated with the discretization of the diffusion terms $\int \frac{\partial \theta}{\partial P} \frac{\partial \varphi_{n+1}}{\partial P} \psi_{\infty}+\int \frac{\partial \theta}{\partial Q} \frac{\partial \varphi_{n+1}}{\partial Q} \psi_{\infty}$ in (5.21) is diagonal. In addition, the use of such a spectral basis allows to circumvent the practical difficulty related to the fact that the equation is posed on the whole space.

\subsection{Microscopic problem: the stochastic approach}

Instead of using the Fokker-Planck equation viewpoint, we may alternatively introduce the couple of stochastic differential equations

$$
\left\{\begin{aligned}
d P(t, y) & =\left(\frac{\partial u}{\partial y}(t, y) Q(t)-\frac{1}{2 \mathrm{We}} P(t, y)\right) d t+\frac{1}{\sqrt{\mathrm{We}}} d V_{t}, \\
d Q(t) & =-\frac{1}{2 \mathrm{We}} Q(t) d t+\frac{1}{\sqrt{\mathrm{We}}} d W_{t},
\end{aligned}\right.
$$


where $V_{t}$ and $W_{t}$ are two independent one-dimensional Brownian motions, and next evaluate the stress with

$$
\tau(t, y)=\frac{\epsilon}{\mathrm{We}} \int_{\mathbb{R}^{2}} P Q \psi(t, y, P, Q) d P d Q=\frac{\epsilon}{\mathrm{We}} \mathbb{E}(P(t, y) Q(t)) .
$$

Note that in this simple geometry and for Hookean dumbbells, $Q(t)$ does not depend on $y$.

In order to solve (5.25), we supply it with initial conditions homogeneous in $y$, and use a forward Euler scheme:

$$
\left\{\begin{array}{l}
P_{i}^{n+1}=\Delta t \frac{U_{i}^{n+1}-U_{i-1}^{n+1}}{\Delta y} Q^{n}+\left(1-\frac{\Delta t}{2 \mathrm{We}}\right) P_{i}^{n}+\sqrt{\frac{\Delta t}{\mathrm{We}}} \Delta V_{i}^{n}, \\
Q^{n+1}=\left(1-\frac{\Delta t}{2 \mathrm{We}}\right) Q^{n}+\sqrt{\frac{\Delta t}{\mathrm{We}}} \Delta W^{n},
\end{array}\right.
$$

for $1 \leq i \leq N$, where $\Delta V_{i}^{n}$ and $\Delta W^{n}$ are standard normal random variables. The stress is then given by

$$
\left(\tau^{h}\right)_{i}^{n+1}=\frac{\epsilon}{\mathrm{We}} \mathbb{E}\left(P_{i}^{n+1} Q^{n+1}\right) .
$$

Following the standard Monte Carlo method, (5.28) is approximated replacing the expectation value by an empirical mean. A supposedly large number $J$ of realizations of the random variables $P_{i}^{n}$ and $Q^{n}$ is generated: (for $1 \leq i \leq N$ )

$$
\begin{aligned}
P_{i, k}^{n+1} & =\Delta t \frac{U_{i}^{n+1}-U_{i-1}^{n+1}}{\Delta y} Q_{k}{ }^{n}+\left(1-\frac{\Delta t}{2 \mathrm{We}}\right) P_{i, k}^{n}+\sqrt{\frac{\Delta t}{\mathrm{We}}} V_{i, k}^{n}, \\
Q_{k}^{n+1} & =\left(1-\frac{\Delta t}{2 \mathrm{We}}\right) Q_{k}^{n}+\sqrt{\frac{\Delta t}{\mathrm{We}}} W_{k}^{n},
\end{aligned}
$$

for $1 \leq k \leq K$, and

$$
\left(\tau^{h}\right)_{i}^{n+1}=\frac{\epsilon}{\mathrm{We}} \frac{1}{K} \sum_{k=1}^{K} P_{i, k}^{n+1} Q_{k}^{n+1}
$$

is computed. For the evolution (5.29) - (5.30), the initial conditions $P_{i}^{0}$ and $Q^{0}$ are chosen as standard normal random variables, since the fluid is assumed at rest at initial time.

This discretization is the CONNFFESSIT approach mentioned above, implemented in a simple case.

A crucial remark is the following. Since the stress $\left(\tau^{h}\right)_{i}^{n+1}$ is an empirical mean (5.31), it is thus also a random variable. It follows that the macroscopic velocity itself, which solves the fully discretized version of (5.11) is a random variable. On the contrary, in the limit $K \rightarrow \infty$, the stress and the velocity are deterministic quantities (since the expectation value (5.28) is a deterministic quantity).

Consequently, when one speaks of computing the velocity or the stress using the stochastic approach, it implies performing a collection of simulations, and averaging over the results. 
Immediately, this brings into the picture variance issues. Let us briefly explain in the present context how the noise inherently present in the numerical simulation may be somewhat reduced. This is the famous variance reduction problem.

A basic approach consists in correlating the trajectories $P_{i}$ from one index $i$ to another one. For this purpose, we first take as initial conditions for $P_{i}$ standard normal random variables $P_{i, k}^{0}=P_{k}^{0}$ that do not depend on $i$, and second use Brownian motions $V_{k}^{n}$, uniform in $i: V_{i, k}^{n}=V_{k}^{n}$. Equation (5.29) is thus replaced with

$$
P_{i, k}^{n+1}=\Delta t \frac{U_{i}^{n+1}-U_{i-1}^{n+1}}{\Delta y} Q_{k}{ }^{n}+\left(1-\frac{\Delta t}{2 \mathrm{We}}\right) P_{i, k}^{n}+\sqrt{\frac{\Delta t}{\mathrm{We}}} V_{k}^{n} .
$$

It is observed that this technique reduces the variance on the velocity $u$. In addition, it provides an empirical mean that is less oscillatory w.r.t. the space variable $y$ than that obtained from the original approach (see Section 6.3.3 below for more details).

Another method, with a large spectrum of applications, is that of control variate. The bottom line is to avoid computing $\mathbb{E}(P Q)$ directly, and to rather compute each of the terms of the sum

$$
\mathbb{E}(P Q)=\mathbb{E}(\tilde{P} \tilde{Q})+\mathbb{E}(P Q-\tilde{P} \tilde{Q})
$$

where $\tilde{P}$ et $\tilde{Q}$ are two processes such that

- $\mathbb{E}(\tilde{P} \tilde{Q})$ is easy to compute or approximate, analytically or numerically,

- $\tilde{P} \tilde{Q}$ is close enough to $P Q$ so that $\operatorname{Var}(P Q-\tilde{P} \tilde{Q}) \ll \operatorname{Var}(P Q)$.

The two extreme situations are

- $\tilde{P}=\tilde{Q}=0$, that is, $\mathbb{E}(\tilde{P} \tilde{Q})$ is very easy to compute but no variance reduction is attained,

- $\tilde{P}=P$ and $\tilde{Q}=Q$, so that $\operatorname{Var}(P Q-\tilde{P} \tilde{Q})=0$ but then $\mathbb{E}(\tilde{P} \tilde{Q})$ is no easier to compute than $\mathbb{E}(P Q)$ !

Somewhat in the style of preconditioners for the resolution of algebraic systems, some compromise has to be found. In the specific case under consideration, an efficient choice consists in defining $(\tilde{P}, \tilde{Q})(t)$ as the solution to the same stochastic differential equations (5.25) for zero velocity and $(\tilde{P}, \tilde{Q})(0)=(P, Q)(0)((\tilde{P}, \tilde{Q})(t)$ remain at equilibrium):

$$
\begin{aligned}
& d \tilde{P}(t)=-\frac{1}{2 \mathrm{We}} \tilde{P}(t) d t+\frac{1}{\sqrt{\mathrm{We}}} d V_{t}, \\
& d \tilde{Q}(t)=-\frac{1}{2 \mathrm{We}} \tilde{Q}(t) d t+\frac{1}{\sqrt{\mathrm{We}}} d W_{t} .
\end{aligned}
$$

Clearly, both $\tilde{Q}$ and $Q$ satisfy the same equation, and $\tilde{P}$ does not depend on $y$. On the other hand, $\mathbb{E}(\tilde{P} \tilde{Q})=0$ since $\tilde{P}$ and $\tilde{Q}$ are independent (since they are at initial time), and both 
of zero mean (arguing on the above stochastic differential equations). In order to simulate $\mathbb{E}(P Q-\tilde{P} \tilde{Q})$, the forward Euler scheme is employed: for each $n$, we set $\tilde{Q}_{k}^{n}=Q_{k}^{n}$ and

$$
\tilde{P}_{i, k}^{n+1}=\left(1-\frac{\Delta t}{2 \mathrm{We}}\right) \tilde{P}_{i, k}^{n}+\sqrt{\frac{\Delta t}{W e}} V_{i, k}^{n} .
$$

Of course, in order for an effective variance reduction to be reached, the same Gaussian variables $V_{i, k}^{n}$ are to be used for simulating both $\tilde{P}$ and $P$. If independent random variables were used for simulating $\tilde{P}$ and $\mathbb{P}, \tilde{P}$ and $\mathbb{P}$ would be independent variables and thus $\operatorname{Var}(P-$ $\tilde{P})=\operatorname{Var}(P)+\operatorname{Var}(\tilde{P})>\operatorname{Var}(P)$.

The simulation of $\left(\tau^{h}\right)_{i}^{n+1}$ consists in solving

$$
\begin{aligned}
\left(\tau^{h}\right)_{i}^{n+1} & =\frac{\epsilon}{\mathrm{We}} \mathbb{E}(P Q), \\
& =\frac{\epsilon}{\mathrm{We}}(\mathbb{E}(\tilde{P} \tilde{Q})+\mathbb{E}(P Q-\tilde{P} \tilde{Q})), \\
& =\frac{\epsilon}{\mathrm{We}}(0+\mathbb{E}(P Q-\tilde{P} \tilde{Q})), \\
& \approx \frac{\epsilon}{\mathrm{We}} \frac{1}{K} \sum_{k=1}^{K}\left(P_{i, k}^{n+1} Q_{k}^{n+1}-\tilde{P}_{i, k}^{n+1} \tilde{Q}_{k}^{n+1}\right), \\
& \approx \frac{\epsilon}{\mathrm{We}} \frac{1}{K} \sum_{k=1}^{K}\left(\left(P_{i, k}^{n+1}-\tilde{P}_{i, k}^{n+1}\right) Q_{k}^{n+1}\right),
\end{aligned}
$$

instead of (5.31).

Summarizing the above, the computation performed at time $t^{n}$, knowing $\left(\left(u^{h}\right)^{n},\left(\tau^{h}\right)^{n}\right)$, in order to advance forward in time $\Delta t$, is:

(1) Knowing all $\left(\tau^{h}\right)_{i}^{n}$ for all intervals indexed by $i$, these values are used in the macroscopic equation (5.12) to obtain the velocity values $U_{i}^{n+1}(1 \leq i \leq N-1)$.

(2) On each space interval with length $\Delta y$,

(2.1) An ensemble of $K$ realizations of the random variables $V_{i, k}^{n}$ and $W_{k}^{n}(1 \leq k \leq$ $K)$ are simulated ; If variance reduction by control variate is used, the random variables $\tilde{P}_{i, k}$ are updated following (5.33);

(2.2) Using the values $U_{i}^{n+1}(1 \leq i \leq N-1)$ in the schemes (5.29)-(5.30) discretizing the SDEs (5.25), the values $P_{i, k}^{n+1}$ and $Q_{k}^{n+1}$ are obtained;

(2.3) By computing the empirical mean (5.31) over the $K$ realizations, the stress $\left(\tau^{h}\right)_{i}^{n+1}$ is obtained at the next timestep. 


\subsection{Extension to the FENE model}

In the FENE model, the SDE that has to be discretized is

$$
d \boldsymbol{X}_{t}+\boldsymbol{u} . \nabla \boldsymbol{X}_{t} d t=\nabla \boldsymbol{u} \boldsymbol{X}_{t} d t-\frac{1}{2 \mathrm{We}} \frac{\boldsymbol{X}_{t}}{1-\left\|\boldsymbol{X}_{t}\right\|^{2} / b} d t+\frac{1}{\sqrt{\mathrm{We}}} d \boldsymbol{W}_{t} .
$$

In the specific geometric setting of this section, denoting $\boldsymbol{X}_{t}=(P(t), Q(t))$ and $\boldsymbol{W}_{t}=$ $\left(V_{t}, W_{t}\right)$, 5.35) writes:

$$
\left\{\begin{aligned}
d P(t, y)= & \left(\frac{\partial u}{\partial y}(t, y) Q(t, y)-\frac{1}{2 \mathrm{We}} \frac{P(t, y)}{1-\left(P(t, y)^{2}+Q(t, y)^{2}\right) / b}\right) d t \\
& +\frac{1}{\sqrt{\mathrm{We}}} d V_{t}, \\
d Q(t, y)= & -\frac{1}{2 \mathrm{We}} \frac{Q(t, y)}{1-\left(P(t, y)^{2}+Q(t, y)^{2}\right) / b} d t+\frac{1}{\sqrt{\mathrm{We}}} d W_{t} .
\end{aligned}\right.
$$

In contrast to the Hookean dumbbell case, notice that $Q$ is now also depending on the space variable $y$.

Let us now discuss how to discretize this SDE, and what type of control variate technique may be employed to reduce the variance.

Compared to the Hookean dumbbell case, an additional difficulty of the discretization of (5.35) is the singularity of the force when $\left\|\boldsymbol{X}_{t}\right\|^{2}$ goes to $b$. It can be shown (see B. Jourdain and T. Lelièvre [66]) that, at the continuous level, the stochastic process $\boldsymbol{X}_{t}$ does not hit the boundary of $\mathcal{B}(0, \sqrt{b})$ in finite time, provided $b>2$. Notice that without the Brownian term, it would be clear that $\boldsymbol{X}_{t}$ remains inside $\mathcal{B}(0, \sqrt{b})$ but this fact is not so clear in the SDE case, and actually requires an assumption on $b$. When discretizing (5.35), one is interested in imposing also this property for the discrete process $\boldsymbol{X}^{n}$. A naïve Euler scheme such as (5.29)-(5.30) does not satisfy this property. One option is to simply reject draws such that $\left|\boldsymbol{X}^{n+1}\right|^{2}>b$. An alternative option has been proposed by H.C. Öttinger [101, p. 218-221]. It consists in treating implicitly the force term, and it can be shown that it yields a discrete process $\boldsymbol{X}^{n}$ with actual values in $\mathcal{B}(0, \sqrt{b})$. Let us write this scheme for the SDE (5.35) without the advection term $\boldsymbol{u} . \nabla \boldsymbol{X}_{t} d t$ :

$$
\left\{\begin{array}{l}
\overline{\boldsymbol{X}^{n+1}}=\boldsymbol{X}^{n}+\nabla \boldsymbol{u}^{n} \boldsymbol{X}^{n} \Delta t-\frac{1}{2 \mathrm{We}} \frac{\boldsymbol{X}^{n}}{1-\left\|\boldsymbol{X}^{n}\right\|^{2} / b} \Delta t+\sqrt{\frac{\Delta t}{\mathrm{We}}} G^{n} \\
\left(1+\frac{1}{4 \mathrm{We}} \frac{\Delta t}{1-\left\|\boldsymbol{X}^{n+1}\right\|^{2} / b}\right) \boldsymbol{X}^{n+1}=\boldsymbol{X}^{n} \\
\quad+\frac{1}{2}\left(\nabla \boldsymbol{u}^{n} \boldsymbol{X}^{n}+\nabla \boldsymbol{u}^{n+1} \overline{\boldsymbol{X}^{n+1}}-\frac{1}{2 \mathrm{We}} \frac{\boldsymbol{X}^{n}}{1-\left\|\boldsymbol{X}^{n}\right\|^{2} / b}\right) \Delta t+\sqrt{\frac{\Delta t}{\mathrm{We}}} G^{n}
\end{array}\right.
$$

where $G^{n}$ are i.i.d. Gaussian variables with covariance matrix Id. 
We next consider the question of variance reduction by control variate. As mentioned above, the idea is to compute the stress tensor as

$$
\boldsymbol{\tau}_{p}=\frac{\epsilon}{\mathrm{We}}\left(\mathbb{E}\left(\frac{\boldsymbol{X}_{t} \otimes \boldsymbol{X}_{t}}{1-\left\|\boldsymbol{X}_{t}\right\|^{2} / b}-\tilde{\boldsymbol{X}}_{t} \otimes \tilde{\boldsymbol{F}}\left(\tilde{\boldsymbol{X}}_{t}\right)\right)+\mathbb{E}\left(\tilde{\boldsymbol{X}}_{t} \otimes \tilde{\boldsymbol{F}}\left(\tilde{\boldsymbol{X}}_{t}\right)\right)\right)
$$

where $\tilde{\boldsymbol{X}}_{t}$ is a suitable chosen stochastic process, and $\tilde{\boldsymbol{F}}$ an adequate force (typically $\tilde{\boldsymbol{F}}=\boldsymbol{F}$ ) such that the variance of the term in the first expectation $\mathbb{E}\left(\frac{\boldsymbol{X}_{t} \otimes \boldsymbol{X}_{t}}{1-\left\|\boldsymbol{X}_{t}\right\|^{2} / b}-\tilde{\boldsymbol{X}}_{t} \otimes \tilde{\boldsymbol{F}}\left(\tilde{\boldsymbol{X}}_{t}\right)\right)$ is as small as possible, and the computation of the second expectation $\mathbb{E}\left(\tilde{\boldsymbol{X}}_{t} \otimes \tilde{\boldsymbol{F}}\left(\tilde{\boldsymbol{X}}_{t}\right)\right)$ is easy. For the variance of the first term to be small, $\tilde{\boldsymbol{X}}_{t}$ needs to be as close as possible to $\boldsymbol{X}_{t}$ (in stochastic terms, $\tilde{\boldsymbol{X}}_{t}$ needs to be coupled to $\boldsymbol{X}_{t}$ ). In particular, one requires that $\boldsymbol{X}_{0}=\tilde{\boldsymbol{X}}_{0}$ and the Brownian motion driving $\boldsymbol{X}_{t}$ is the same as the one driving $\tilde{\boldsymbol{X}}_{t}$.

Then two types of control variate are classically used (see J. Bonvin and M. Picasso [16]). As in the previous section for Hookean dumbbells, $\tilde{\boldsymbol{X}}_{t}$ can be the process "at equilibrium". It consists in computing $\tilde{\boldsymbol{X}}_{t}$ as the solution to the same SDE as $\boldsymbol{X}_{t}$ (and thus $\tilde{\boldsymbol{F}}=\boldsymbol{F}$ ) without the term $\nabla \boldsymbol{u} \boldsymbol{X}_{t} d t$. If $\boldsymbol{X}_{0}=\tilde{\boldsymbol{X}}_{0}$ is distributed according to the invariant law of the $\mathrm{SDE}$, then the law of $\tilde{\boldsymbol{X}}_{t}$ does not depend on time and thus

$$
\mathbb{E}\left(\frac{\tilde{\boldsymbol{X}}_{t} \otimes \tilde{\boldsymbol{X}}_{t}}{1-\left\|\tilde{\boldsymbol{X}}_{t}\right\|^{2} / b}\right)=\mathbb{E}\left(\frac{\tilde{\boldsymbol{X}}_{0} \otimes \tilde{\boldsymbol{X}}_{0}}{1-\left\|\tilde{\boldsymbol{X}}_{0}\right\|^{2} / b}\right)
$$

which can be analytically computed. This method typically works when the system remains close to equilibrium.

When the system goes out of equilibrium, another idea is to use a closure approximation to obtain a model which is close to the FENE model, but which has a macroscopic equivalent so that the second term $\mathbb{E}\left(\tilde{\boldsymbol{X}}_{t} \otimes \tilde{\boldsymbol{F}}\left(\tilde{\boldsymbol{X}}_{t}\right)\right)$ can be computed by discretizing a PDE (which is very cheap compared to the Monte Carlo method). For example, one can take the Hookean dumbbell model $\left(\tilde{\boldsymbol{F}}\left(\tilde{\boldsymbol{X}}_{t}\right)=\tilde{\boldsymbol{X}}_{t}\right)$ and compute $\mathbb{E}\left(\tilde{\boldsymbol{X}}_{t} \otimes \tilde{\boldsymbol{X}}_{t}\right)$ by solving the PDE for the Oldroyd-B model. One can also choose the FENE-P model $\left(\tilde{\boldsymbol{F}}\left(\tilde{\boldsymbol{X}}_{t}\right)=\frac{\tilde{\boldsymbol{X}}_{t} \otimes \tilde{\boldsymbol{X}}_{t}}{1-\mathbb{E}\left\|\tilde{\boldsymbol{X}}_{t}\right\|^{2} / b}\right)$ and compute $\mathbb{E}\left(\tilde{\boldsymbol{X}}_{t} \otimes \tilde{\boldsymbol{F}}\left(\tilde{\boldsymbol{X}}_{t}\right)\right)$ by solving the associated PDE (2.28). Closure relations are thus important not only to obtain macroscopic models with microscopic interpretation, but also to build efficient variance reduction methods. For closure relations for the FENE model, we refer to Q. Du, C. Liu and P. Yu [37, 32].

\subsection{Matlab codes}

In this section, we give the Matlab codes for the computation of the velocity and the stress in a Couette flow for the Hookean dumbbell model (start-up of shear flow). We recall that this model is equivalent to the Oldroyd-B model. We thus have three formulations of the problem: 
- The macro-macro formulation:

$$
\left\{\begin{array}{l}
\operatorname{Re} \frac{\partial u}{\partial t}(t, y)-(1-\epsilon) \frac{\partial^{2} u}{\partial y^{2}}(t, y)=\frac{\partial \tau}{\partial y}(t, y), \\
\frac{\partial \tau}{\partial t}+\frac{1}{\mathrm{We}} \tau=\frac{\epsilon}{\mathrm{We}} \frac{\partial u}{\partial y}
\end{array}\right.
$$

- The micro-macro formulation with the SDEs:

$$
\left\{\begin{array}{l}
\operatorname{Re} \frac{\partial u}{\partial t}(t, y)-(1-\epsilon) \frac{\partial^{2} u}{\partial y^{2}}(t, y)=\frac{\partial \tau}{\partial y}(t, y) \\
\tau(t, y)=\frac{\epsilon}{\mathrm{We}} \mathbb{E}\left(X_{t}(y) Y_{t}\right) \\
d X_{t}(y)=\frac{\partial u}{\partial y}(t, y) Y_{t} d t-\frac{1}{2 \mathrm{We}} X_{t}(y) d t+\frac{1}{\sqrt{\mathrm{We}}} d V_{t} \\
d Y_{t}=-\frac{1}{2 \mathrm{We}} Y_{t} d t+\frac{1}{\sqrt{\mathrm{We}}} d W_{t}
\end{array}\right.
$$

- The micro-macro formulation with the Fokker-Planck equation:

$$
\left\{\begin{array}{l}
\operatorname{Re} \frac{\partial u}{\partial t}(t, y)-(1-\epsilon) \frac{\partial^{2} u}{\partial y^{2}}(t, y)=\frac{\partial \tau}{\partial y}(t, y) \\
\tau(t, y)=\frac{\epsilon}{\mathrm{We}} \int X Y p(t, y, X, Y) d X d Y \\
\frac{\partial p}{\partial t}=-\operatorname{div}_{(X, Y)}\left(\left(\left(\frac{\partial u}{\partial y} Y, 0\right)-(X, Y) \frac{1}{2 \mathrm{We}}\right) p\right)+\frac{1}{2 \mathrm{We}} \Delta_{(X, Y)} p .
\end{array}\right.
$$

We now insert the Matlab source Couette_0ldroyd_B.m for the discretization of (5.38).

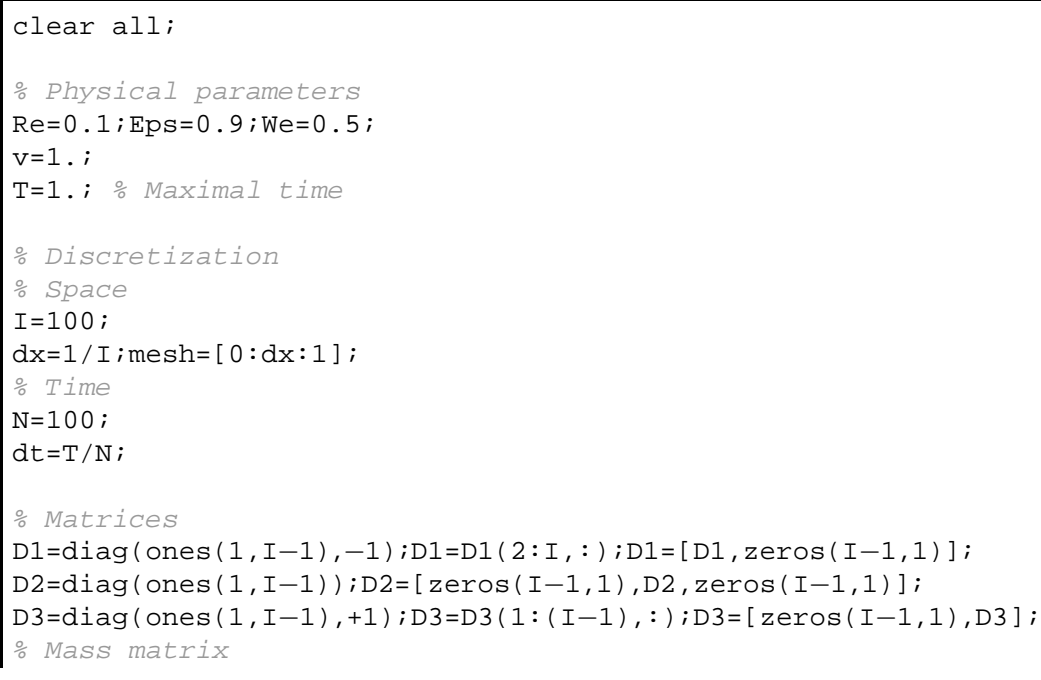




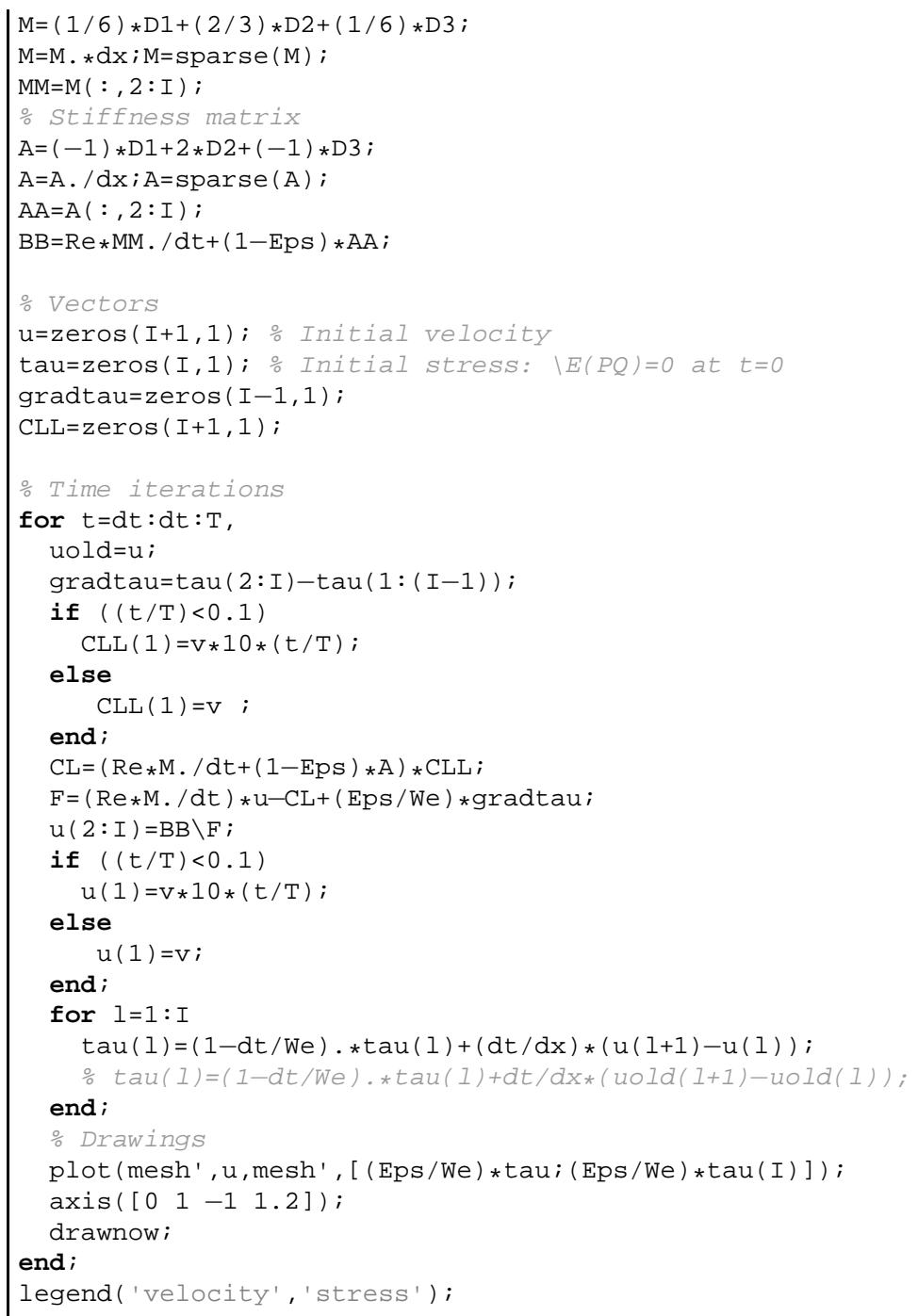

Exercise 1 Compare numerically and theoretically the stability of the two time-discretizations:

$$
\left\{\begin{array}{l}
\frac{\operatorname{Re}}{\delta t}\left(u_{n+1}(y)-u_{n}(y)\right)-(1-\epsilon) \frac{\partial^{2} u_{n+1}}{\partial y^{2}}(y)=\frac{\partial \tau_{n}}{\partial y}(y) \\
\frac{1}{\delta t}\left(\tau_{n+1}(y)-\tau_{n}(y)\right)+\frac{1}{\mathrm{We}} \tau_{n+1}(y)=\frac{\epsilon}{\mathrm{We}} \frac{\partial u_{n+1}}{\partial y}
\end{array}\right.
$$


and

$$
\left\{\begin{array}{l}
\frac{\operatorname{Re}}{\delta t}\left(u_{n+1}(y)-u_{n}(y)\right)-(1-\epsilon) \frac{\partial^{2} u_{n+1}}{\partial y^{2}}(y)=\frac{\partial \tau_{n}}{\partial y}(y), \\
\frac{1}{\delta t}\left(\tau_{n+1}(y)-\tau_{n}(y)\right)+\frac{1}{\mathrm{We}} \tau_{n+1}(y)=\frac{\epsilon}{\mathrm{We}} \frac{\partial u_{n}}{\partial y}
\end{array}\right.
$$

Hint: For the numerics, choose a sufficiently large timestep. For the numerical analysis, consider the quantity $E_{n}=\operatorname{Re} \int_{0}^{1}\left|u_{n}\right|^{2}(y) d y+\frac{\mathrm{We}}{\epsilon} \int_{0}^{1}\left|\tau_{n}\right|^{2}(y) d y$ and prove that $E_{n+1} \leq E_{n}$, whatever the timestep for the scheme (5.41), and for a sufficiently small timestep for the scheme (5.42). The scheme (5.41) (resp. (5.42)) is thus inconditionally (resp. conditionally) stable.

Below is the Matlab source Couette_MC_VarReduc.m for the discretization of (5.39).

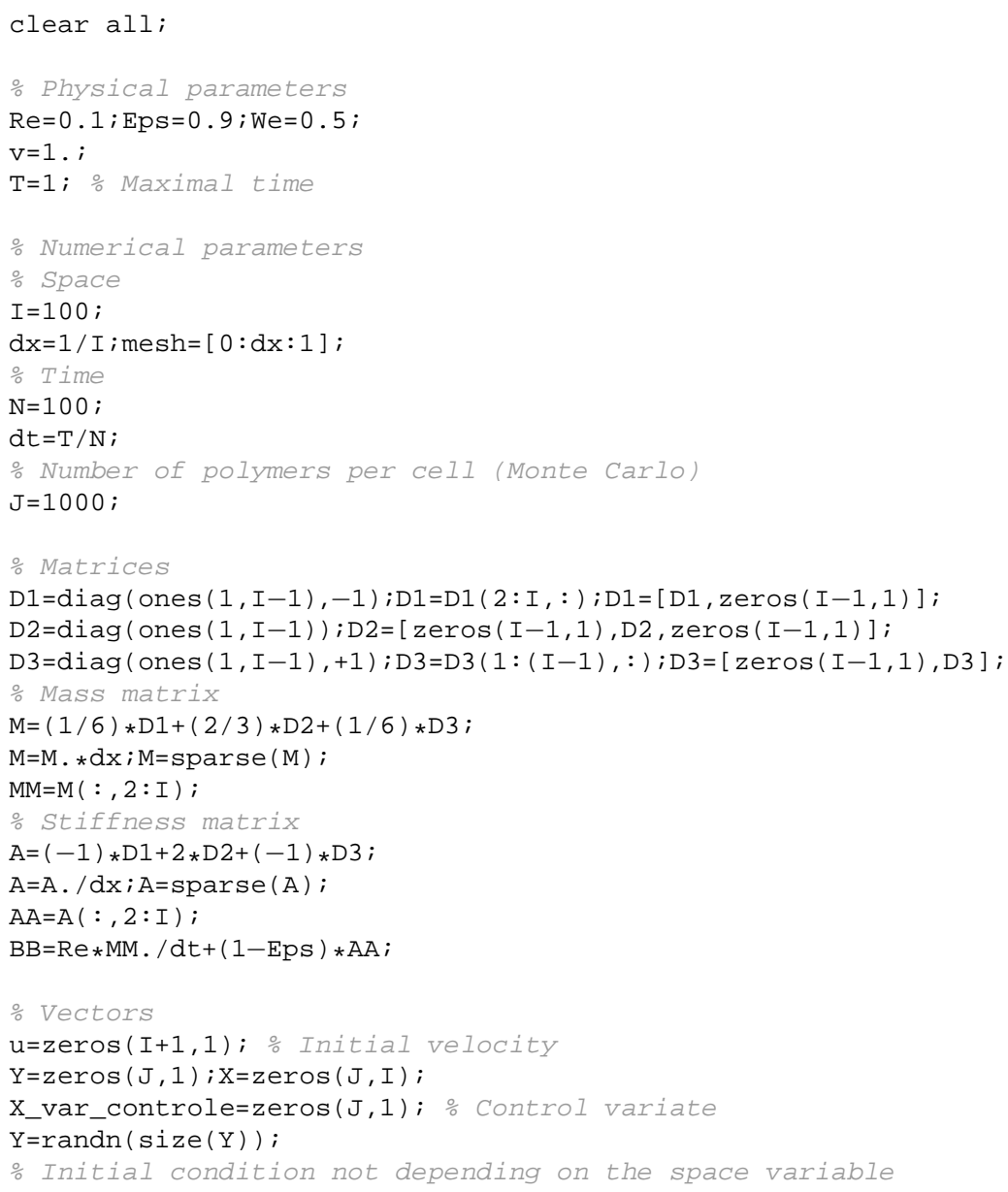




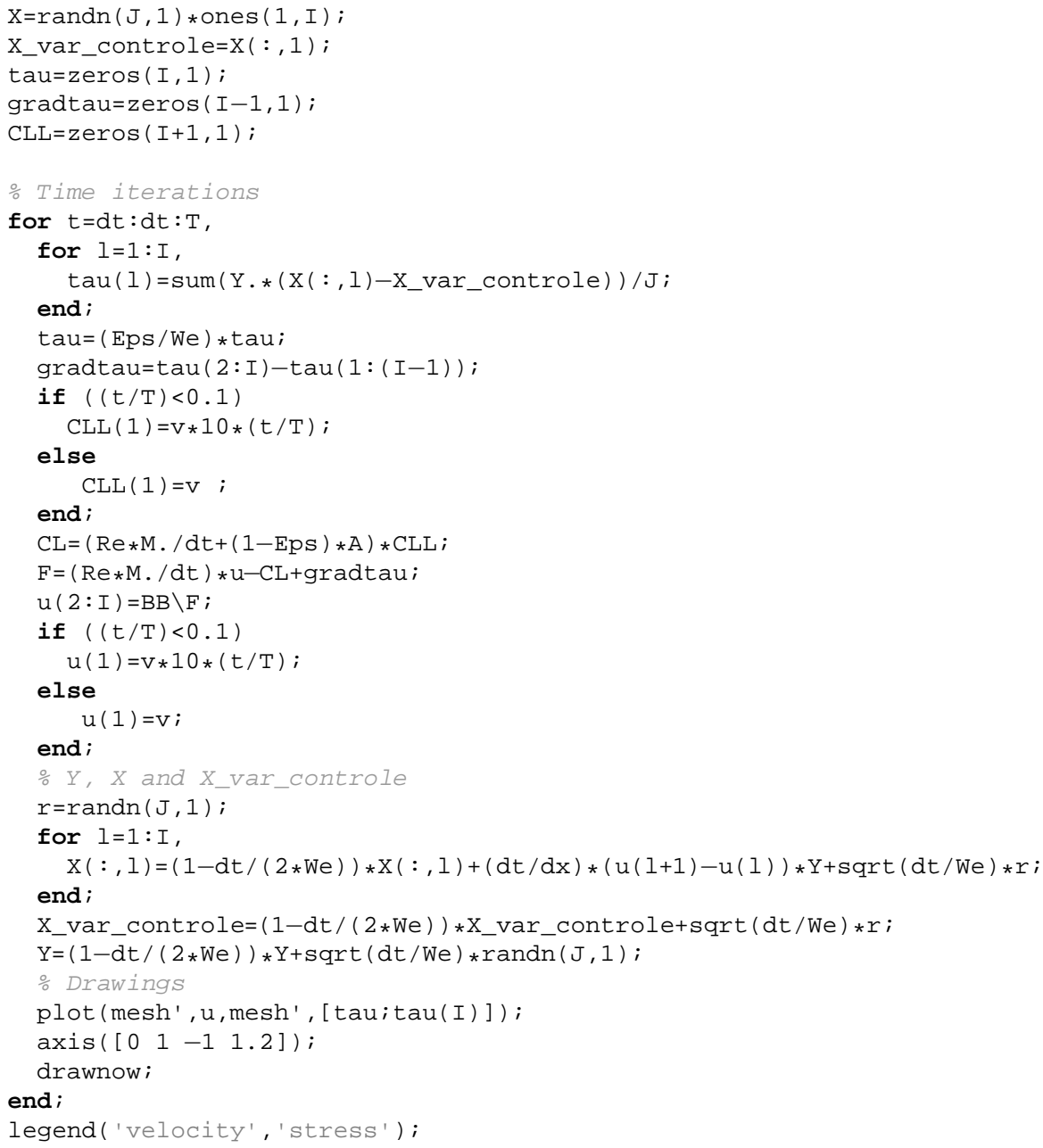

Exercise 2 Investigate numerically the influence of the number of dumbbells in each cell. Compare the results with and without variance reduction. Modify the program to use Brownian motions $V_{t}$ for $X_{t}$ which are independent from one cell to another (again with and without variance reduction). Discuss the results (see Section [6.3.3 below).

Exercise 3 Modify the program to treat FENE dumbbells. You can use either an Euler scheme to discretize the SDE and a rejection step, or the scheme (5.37). Program a variance reduction using the FENE-P model for the control variate.

The Matlab source Couette_FP.m for the discretization of (5.40) follows. 


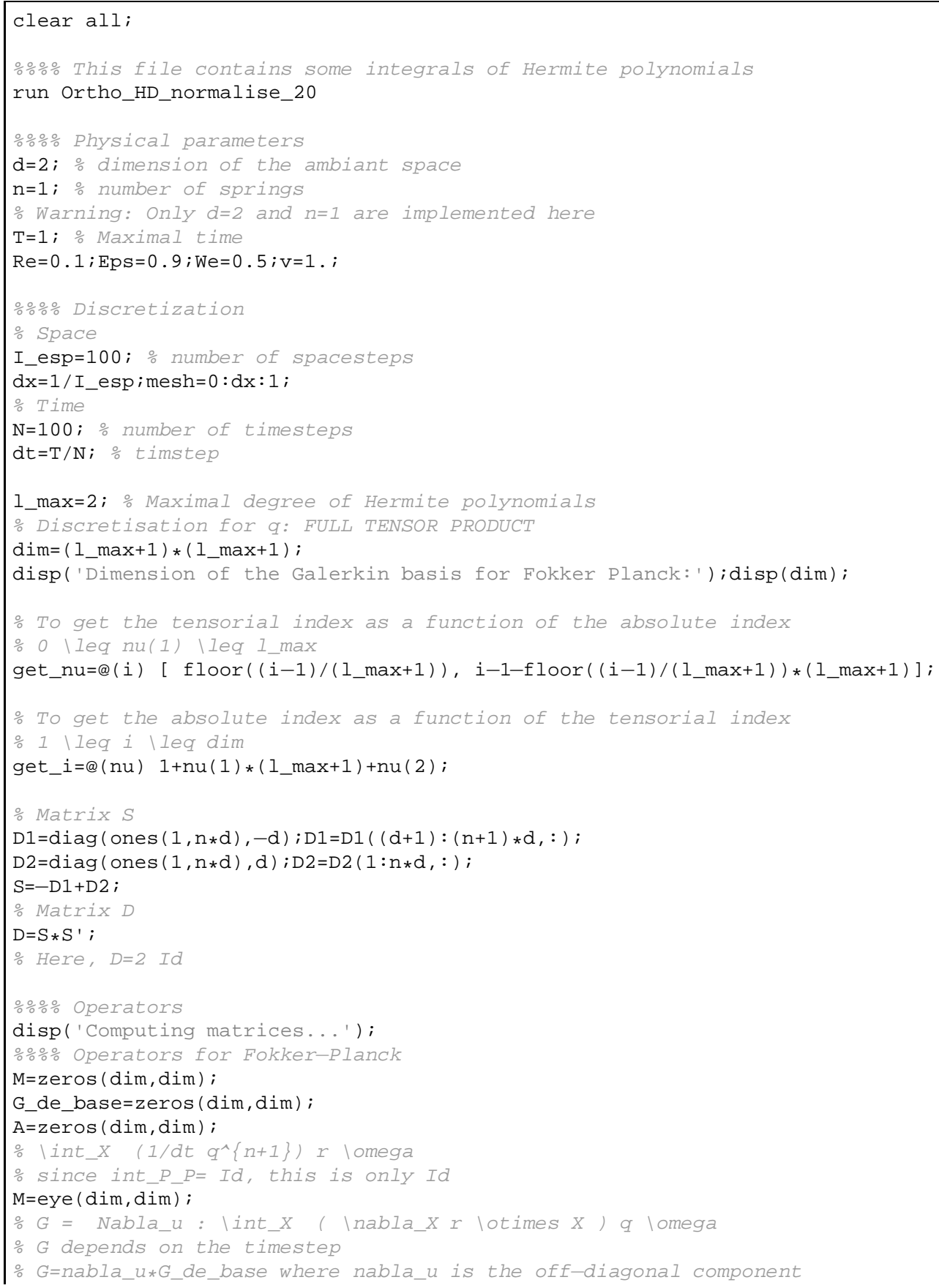




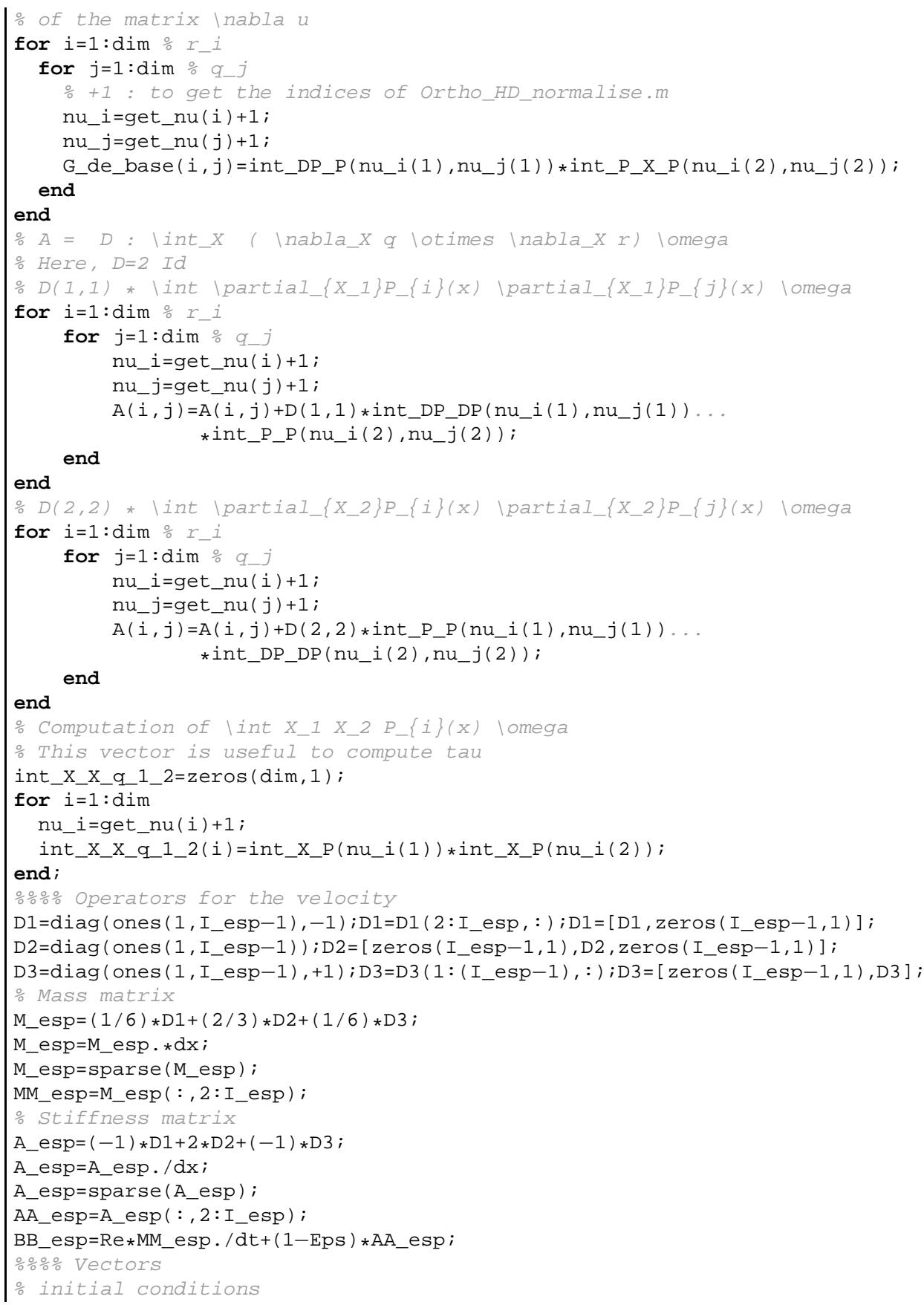




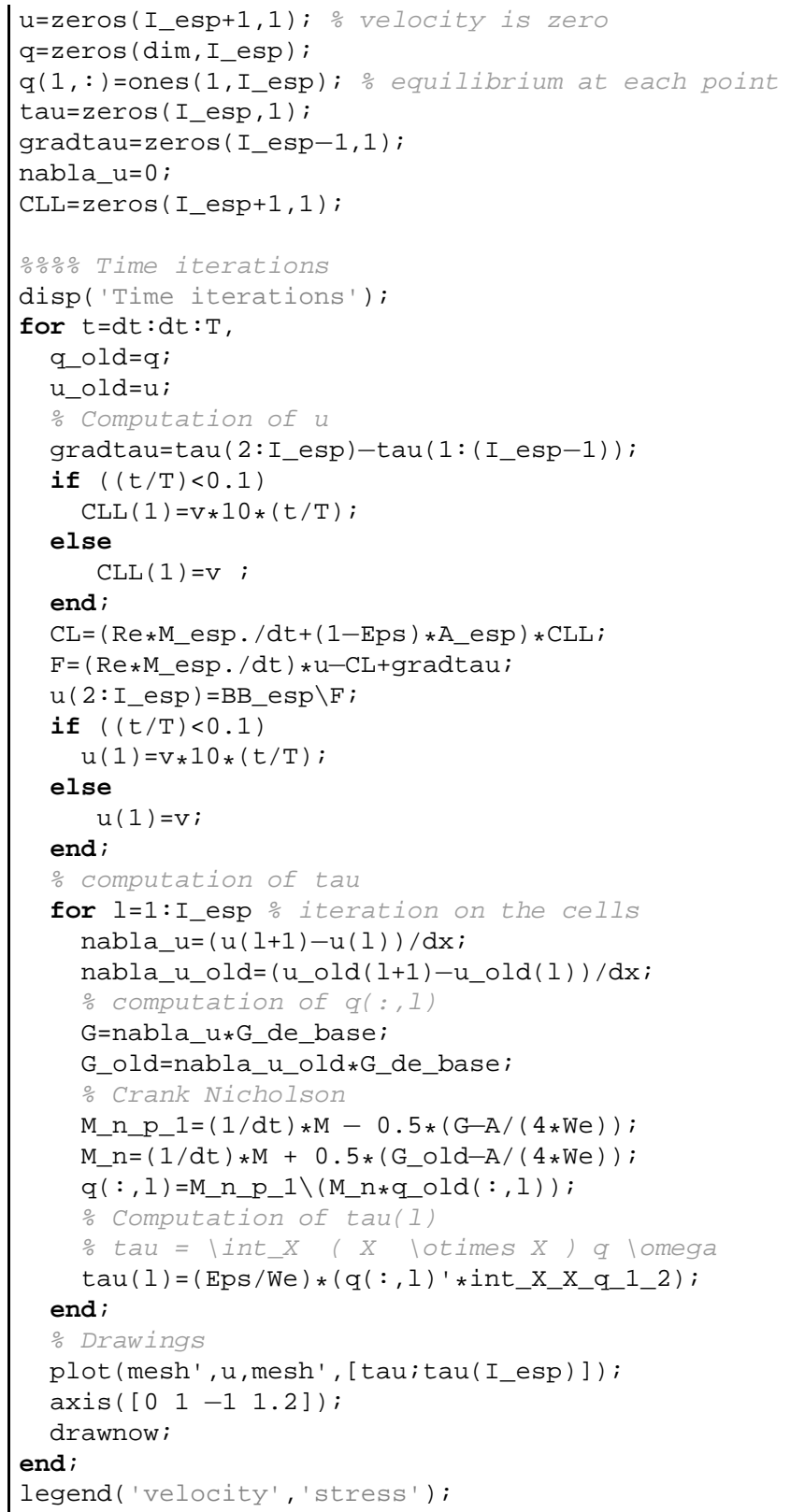

Exercise 4 Compare the results obtained with the three formulations. Which formulation is the most efficient computationally? Discuss the applicability of these three formulations to 
the following two more general settings: chain of $N>2$ beads linked with Hookean springs, FENE dumbbell model.

\section{$6 \quad$ Mathematical and numerical issues}

As mentioned earlier, the present section is much more elaborate mathematically than the preceeding sections.

\subsection{Overview of the main difficulties}

Let us first formally summarize the difficulties raised by the mathematical analysis of systems such as (3.14) and (4.46) (for micro-macro models) or (2.23) (for macro-macro models).

These systems of equations include the Navier-Stokes equations, with the additional term $\operatorname{div} \boldsymbol{\tau}_{p}$ in the right-hand side. The equation on $\boldsymbol{\tau}_{p}$ is essentially a transport equation and, formally, $\boldsymbol{\tau}_{p}$ has at most the regularity of $\nabla \boldsymbol{u}$ (this fact will be clear in the choice of appropriate functional spaces for existence results, and of the discretization spaces for numerical methods). The term $\operatorname{div} \tau_{p}$ in the right-hand side in the momentum equation is not likely to bring more regularity on $\boldsymbol{u}$. It is thus expected that the study of these coupled systems contains at least the well-known difficulties of the Navier-Stokes equations. Recall that for the (3-dimensional) Navier-Stokes equations, it is known that global-in-time weak solutions exist but the regularity, and thus the uniqueness, of such solutions is an open problem. Only local-in-time existence and uniqueness results of strong solutions are available.

In addition to the difficulties already contained in the Navier-Stokes equations (which essentially originate from the Navier term $\boldsymbol{u} \cdot \nabla \boldsymbol{u}$ ), the coupling with the equation on $\boldsymbol{\tau}_{p}$ raises other problems. First, these equations (both for macro-macro and micro-macro models) contain a transport term $\left(\boldsymbol{u} \cdot \nabla \boldsymbol{\tau}_{p}, \boldsymbol{u} \cdot \nabla \psi\right.$ or $\left.\boldsymbol{u} \cdot \nabla \boldsymbol{X}_{t}\right)$ without diffusion terms (in the space variable). They are hyperbolic in nature. The regularity on the velocity $\boldsymbol{u}$ is typically not sufficient to treat this transport term by a characteristic method. Moreover, these equations involve a nonlinear multiplicative term $\left(\nabla \boldsymbol{u} \nabla \boldsymbol{\tau}_{p}\right.$, div $\boldsymbol{X}(\nabla \boldsymbol{u} \boldsymbol{X} \nabla \psi)$ or $\left.\nabla \boldsymbol{u} \boldsymbol{X}_{t}\right)$. Finally, except for very simple models (Oldroyd-B or Hookean dumbbell), the equations defining $\boldsymbol{\tau}_{p}$ generally contain additional non-linearities (for micro-macro model, the force $\boldsymbol{F}$ is generally non-linear and typically blows up when the length of the polymer reaches a critical value).

To summarize, the difficulties raised by mathematical analysis of these models are related to:

- transport terms

- nonlinear terms coming either from the coupling between the equations and $(\boldsymbol{u}, p)$ and $\tau_{p}$, or inherently contained in the equations defining $\tau_{p}$.

These difficulties limit the state-of-the-art mathematical well-posedness analysis to local-intime existence and uniqueness results. They also have many implications on the numericals 
methods (choice of the discretization spaces, stability of the numerical schemes, ...). Actually, the problems raised by the discretization we mentioned in Section 4.4 .2 can be seen as counterparts of the difficulties raised by the mathematical analysis. Many questions are still open, and the mathematical analysis and the numerical analysis for viscoelastic fluids are very lively fields.

In the following, we provide more detailed results for macro-macro models, and, next, micro-macro models. Considering the focus of the present article, more emphasis is laid on the latter.

\subsection{Macroscopic models}

We refer to M. Renardy [111] or E. Fernandez-Cara, F. Guillen and R.R. Ortega [44] for a review of the mathematical analysis of macroscopic models. For the numerical methods, we refer to R. Keunings [70] F.P.T. Baaijens [6] R. Owens and T. Phillips [103]. We recall the prototypical macroscopic model, namely the Oldroyd-B model:

$$
\left\{\begin{array}{l}
\operatorname{Re}\left(\frac{\partial \boldsymbol{u}}{\partial t}+\boldsymbol{u} \cdot \nabla \boldsymbol{u}\right)-(1-\epsilon) \Delta \boldsymbol{u}+\nabla p=\operatorname{div} \boldsymbol{\tau}_{p}+\boldsymbol{f} \\
\operatorname{div} \boldsymbol{u}=0 \\
\operatorname{We}\left(\frac{\partial \boldsymbol{\tau}_{p}}{\partial t}+\boldsymbol{u} \cdot \nabla \boldsymbol{\tau}_{p}-\nabla \boldsymbol{u} \boldsymbol{\tau}_{p}-\boldsymbol{\tau}_{p}(\nabla \boldsymbol{u})^{T}\right)+\boldsymbol{\tau}_{p}=\epsilon\left(\nabla \boldsymbol{u}+\nabla \boldsymbol{u}^{T}\right)
\end{array}\right.
$$

\subsubsection{Mathematical results}

Concerning existence results for macroscopic models, four types of results can be found in the litterature:

- local-in-time results (perturbation of the initial condition),

- global-in-time results for small data (perturbation of the stationary solution),

- existence results for stationary solutions close to equilibrium solutions,

- existence results for stationary solutions close to Navier-Stokes stationary solutions.

For illustration, let us only mention the result obtained by M. Renardy in [109. The author considers the following coupled problem, in a bounded domain $\mathcal{D}$ of $\mathbb{R}^{3}$ :

$$
\left\{\begin{array}{l}
\rho\left(\frac{\partial \boldsymbol{u}}{\partial t}+\boldsymbol{u} . \nabla \boldsymbol{u}\right)=\operatorname{div} \boldsymbol{\tau}_{p}-\nabla p+\boldsymbol{f} \\
\operatorname{div} \boldsymbol{u}=0 \\
\left(\frac{\partial}{\partial t}+\boldsymbol{u} . \nabla\right)\left(\boldsymbol{\tau}_{p}\right)_{i, j}=\boldsymbol{A}_{i, j, k, l}\left(\boldsymbol{\tau}_{p}\right) \frac{\partial \boldsymbol{u}_{k}}{\partial \boldsymbol{x}_{l}}+\boldsymbol{g}_{i, j}\left(\boldsymbol{\tau}_{p}\right)
\end{array}\right.
$$

with summation convention on repeated indices. The fluid is inviscid $(\eta=0)$. This system is supplied with homogeneous Dirichlet boundary condition on the velocity $\boldsymbol{u}$, and initial 
conditions. The differential models introduced in Section 2.3.1 indeed enter this framework. Introduce the fourth order tensor:

$$
\boldsymbol{C}_{i, j, k, l}=\boldsymbol{A}_{i, j, k, l}-\left(\boldsymbol{\tau}_{p}\right)_{i, l} \delta_{k, j},
$$

where $\delta$ is the Kronecker symbol. Assume the following strong ellipticity property on $C$ : $\forall \zeta, \eta \in \mathbb{R}^{3}$

$$
\boldsymbol{C}_{i, j, k, l}\left(\boldsymbol{\tau}_{p}\right) \zeta_{i} \zeta_{k} \eta_{j} \eta_{l} \geq \kappa|\zeta|^{2}|\eta|^{2}
$$

where $\kappa>0$ is a constant not depending on $\boldsymbol{\tau}_{p}$. Under additional assumptions of symmetry on the tensor $\boldsymbol{A}$, of regularity and compatibility on the initial conditions, it is shown by M. Renardy in [109] that:

Theorem 1 There exists a time $T^{\prime}>0$, such that the system (6.2) admits a unique solution with regularity:

$$
\boldsymbol{u} \in \bigcap_{k=0}^{4} \mathcal{C}^{k}\left(\left[0, T^{\prime}\right], H^{4-k}(\mathcal{D}), \boldsymbol{\tau}_{p} \in \bigcap_{k=0}^{3} \mathcal{C}^{k}\left(\left[0, T^{\prime}\right], H^{3-k}(\mathcal{D}) .\right.\right.
$$

The works of C. Guillopé and J.C. Saut [53, 54] are also to be mentioned. Existence results for less regular solutions are obtained there for non-zero viscosity of the solvent $\eta>0$. In a series of works, E. Fernandez-Cara, F. Guillen and R.R. Ortega study the local well-posedness in Sobolev spaces (see [44] and references therein).

We also mention the work of F. Lin, C. Liu and P.W. Zhang [86] where local-in-time existence and uniqueness results and global-in-time existence and uniqueness results for small data are proven for Oldroyd-like models.

The only global-in-time existence result we are aware of is the work of P.-L. Lions and N. Masmoudi [88] where an Oldroyd-like model is studied, but with the corotational convective derivative on the stress tensor rather than the upper convected derivative.

Besides, there exists many studies on the stability of viscoelastic flows, and the change of mathematical nature of the equations (transition from parabolic to hyperbolic). We refer to M. Renardy [111], R. Owens and T. Phillips [103] and references therein.

\subsubsection{Numerical methods}

Most of the numerical methods employed in practice to simulate such models are based upon a finite element discretization in space (see however R. Owens and T. Phillips [103] for spectral methods) and a finite difference discretization in time (usually Euler schemes), with a decoupled computation of $(\boldsymbol{u}, p)$ and $\boldsymbol{\tau}_{p}$. More precisely, at each timestep, the equation for $(\boldsymbol{u}, p)$ is first solved, given the current stress tensor $\boldsymbol{\tau}_{p}$. This allows to update the velocity. Next, the equation for $\tau_{p}$ is solved, and the stress is updated.

We have already mentioned in Section 4.4.2 the main three difficulties raised by the discretization: (i) a compatibility condition is needed between the discretization spaces for $\boldsymbol{u}$ and for $\boldsymbol{\tau}_{p}$, (ii) the transport terms need to be correctly discretized, (iii) the discretization 
of the nonlinear terms require special attention. Let us now briefly describe how to deal with these difficulties for macroscopic models. Notice that, as observed in Section 4.4.2, the three difficulties mentioned above are also present for the discretization of micro-macro models. Most of the methods described below are thus also useful for the discretization of micro-macro models.

Concerning difficulty (i), it actually appears that an inf-sup condition is required for the three discretization spaces for respectively the pressure, the velocity and the stress tensor. More precisely, in addition to the usual inf-sup condition required for the discretization spaces for the velocity and the pressure, a compatibility between the discretization space for the velocity and that for the stress tensor is required to obtain stable schemes when $\eta$ is small as compared to $\eta_{p}$ (i.e. when $\epsilon$ is close to 1 ). These compatibility conditions have been analyzed by J.C. Bonvin M. Picasso and R. Sternberg in [18, 17] on the three-field Stokes system:

$$
\left\{\begin{array}{l}
-\eta \Delta \boldsymbol{u}+\nabla p-\operatorname{div} \boldsymbol{\tau}_{p}=\boldsymbol{f} \\
\operatorname{div} \boldsymbol{u}=0 \\
\boldsymbol{\tau}_{p}-\eta_{p} \dot{\boldsymbol{\gamma}}=\boldsymbol{g} .
\end{array}\right.
$$

Many methods have been proposed in the literature to treat the problem:

- Use discretization spaces that satisfy an inf-sup condition. These are usually difficult to implement (see for example J.M. Marchal and M.J. Crochet [95]),

- Introduce an additional unknown to avoid this compatibility condition (see the EVSS method in R. Guénette and M. Fortin [52]),

- Use stabilization methods, like the Galerkin Least Square (GLS) method, which enables to use the same discretization space for the three unknown fields (see J.C. Bonvin M. Picasso and R. Sternberg in [18, 17]).

The second difficulty (ii) is raised by the discretization of the advection terms both in the equation for $\boldsymbol{u}$ and for $\boldsymbol{\tau}_{p}$. It is well known that naïve discretization by a finite element method leads to unstable schemes. Many techniques have been used to circumvent this problem: stabilization techniques like Streamline Upwind Petrov-Galerkin (SUPG) or GLS, Discontinuous Galerkin methods (see M. Fortin and A. Fortin [46]), or numerical characteristic method (see J.C. Bonvin [18] or the Backward-tracking Lagrangian Particle Method of P. Wapperom, R. Keunings and V. Legat [120]). We refer to R. Owens and T. Phillips [103, Chapter 7] or to R. Keunings [71] for references about these methods in the context of viscoelastic fluid simulations (see also T. Min, J.Y. Yoo and H. Choi [97] for a comparison between various numerical schemes). These difficulties are prominent for high Reynolds number (which is not practically relevant in the context of viscoelastic fluid simulations) or for high Weissenberg number (which is relevant).

The third difficulty (iii) we mentioned concerns the discretization of the nonlinear terms. Consider the term $\nabla \boldsymbol{u} \boldsymbol{\tau}_{p}+\boldsymbol{\tau}_{p}(\nabla \boldsymbol{u})^{T}$ in the convective derivative of $\boldsymbol{\tau}_{p}$. In most of the numerical methods, this term is treated explicitly by taking its value at the former timestep. 
Linearizing this term by treating the velocity explicitly and the stress implicitly leads to an ill-posed problem if the Weissenberg problem is too high.

We mentioned that two of these difficulties are prominent for large Weissenberg number. It indeed appears that numerical methods become unstable in this latter regime. This is the so-called High Weissenberg Number Problem (HWNP) we already mentioned in Section 4.4.2 Many works are related to the HWNP (we refer for example to R. Owens and T. Phillips [103, Chapter 7]). The HWNP is certainly not only related to the discretization scheme. It has indeed been observed that for some geometries, the critical Weissenberg number (above which the scheme is unstable) decreases with the mesh step size (see R. Keunings [71]), which could indicate a loss of regularity for the continuous solution itself (see D. Sandri [115]). It is still an open problem to precisely characterize the HWNP, and to distinguish between instability coming from the model itself, or its discretization. For the theoretical study of the limit We $\rightarrow \infty$, we refer to M. Renardy [111, Chapter 6].

We would like to mention the recent works [42, 43, 60] where R. Fattal, R. Kupferman and M.A. Hulsen propose a new formulation for macroscopic models based on a change of variable: instead of using $\left(\boldsymbol{u}, p, \boldsymbol{\tau}_{p}\right)$ as unknowns, they set the problem in terms of $(\boldsymbol{u}, p, \boldsymbol{\phi})$, where

$$
\phi=\ln A
$$

and $\boldsymbol{A}$ is the conformation tensor defined by:

$$
\boldsymbol{A}=\frac{\mathrm{We}}{\epsilon} \boldsymbol{\tau}_{p}+\mathrm{Id}
$$

This new formulation was implemented in R. Fattal, R. Kupferman and M.A. Hulsen [43, 60 and Y. Kwon [74] for various models, various geometric settings, and various numerical methods. In this alternate formulation, the numerical instability arises only for much higher a Weissenberg number. It thus seems to be a promising method to better understand the problem.

\subsection{Multiscale models}

Let us recall the micro-macro model we are interested in:

$$
\left\{\begin{array}{l}
\operatorname{Re}\left(\frac{\partial \boldsymbol{u}}{\partial t}+\boldsymbol{u} \cdot \nabla \boldsymbol{u}\right)-(1-\epsilon) \Delta \boldsymbol{u}+\nabla p=\operatorname{div} \boldsymbol{\tau}_{p}+\boldsymbol{f} \\
\operatorname{div} \boldsymbol{u}=0 \\
\boldsymbol{\tau}_{p}=\frac{\epsilon}{\mathrm{We}}\left(\mathbb{E}\left(\boldsymbol{X}_{t} \otimes \boldsymbol{F}\left(\boldsymbol{X}_{t}\right)\right)-\mathrm{Id}\right) \\
d \boldsymbol{X}_{t}+\boldsymbol{u} \cdot \nabla \boldsymbol{X}_{t} d t=\nabla \boldsymbol{u} \boldsymbol{X}_{t} d t-\frac{1}{2 \mathrm{We}} \boldsymbol{F}\left(\boldsymbol{X}_{t}\right) d t+\frac{1}{\sqrt{\mathrm{We}}} d \boldsymbol{W}_{t} .
\end{array}\right.
$$

with $\boldsymbol{F}\left(\boldsymbol{X}_{t}\right)=\boldsymbol{X}_{t}$ for Hookean dumbbells, $\boldsymbol{F}\left(\boldsymbol{X}_{t}\right)=\frac{\boldsymbol{X}_{t}}{1-\left\|\boldsymbol{X}_{t}\right\|^{2} / b}$ for FENE dumbbells, or $\boldsymbol{F}\left(\boldsymbol{X}_{t}\right)=\frac{\boldsymbol{X}_{t}}{1-\mathbb{E}\left(\left\|\boldsymbol{X}_{t}\right\|^{2}\right) / b}$ for FENE-P dumbbells. The space variable $\boldsymbol{x}$ varies in a bounded domain $\mathcal{D} \subset \mathbb{R}^{d}$. This system is supplied with boundary conditions on the velocity, and 
initial conditions on the velocity and the stochastic processes. In the following, we suppose $\epsilon \in(0,1)$.

We recall the Fokker-Planck version of (6.7):

$$
\left\{\begin{array}{l}
\operatorname{Re}\left(\frac{\partial \boldsymbol{u}}{\partial t}(t, \boldsymbol{x})+\boldsymbol{u}(t, \boldsymbol{x}) \cdot \nabla \boldsymbol{u}(t, \boldsymbol{x})\right)-(1-\epsilon) \Delta \boldsymbol{u}(t, \boldsymbol{x})+\nabla p(t, \boldsymbol{x}) \\
\quad=\operatorname{div}\left(\boldsymbol{\tau}_{p}(t, \boldsymbol{x})\right) \\
\operatorname{div}(\boldsymbol{u}(t, \boldsymbol{x}))=0 \\
\boldsymbol{\tau}_{p}(t, \boldsymbol{x})=\frac{\epsilon}{\mathrm{We}}\left(\int_{\boldsymbol{X}}(\boldsymbol{X} \otimes \boldsymbol{F}(\boldsymbol{X})) \psi(t, \boldsymbol{x}, \boldsymbol{X}) d \boldsymbol{X}-\mathrm{Id}\right) \\
\frac{\partial \psi}{\partial t}(t, \boldsymbol{x}, \boldsymbol{X})+\boldsymbol{u} \cdot \nabla_{\boldsymbol{x}} \psi(t, \boldsymbol{x}, \boldsymbol{X}) \\
\quad=-\operatorname{div}_{\boldsymbol{X}}\left(\left(\nabla \boldsymbol{u}(t, \boldsymbol{x}) \boldsymbol{X}-\frac{1}{2 \mathrm{We}} \boldsymbol{F}(\boldsymbol{X})\right) \psi(t, \boldsymbol{x}, \boldsymbol{X})\right)+\frac{1}{2 \mathrm{We}} \Delta_{\boldsymbol{X}} \psi(t, \boldsymbol{x}, \boldsymbol{X}) .
\end{array}\right.
$$

There is a growing literature on the analysis of micro-macro models for polymeric fluids. The first work we are aware of is M. Renardy [110, where the micro-macro model in its Fokker-Planck formulation (3.14) is studied. Since this early work, many groups have studied these models, perhaps because they are prototypical for a class of multiscale models, where some parameters needed in the macroscopic equations are computed by some microscopic models (see the general formulation (3.16)).

Let us recall the two main difficulties we already mentioned in Section 6.1,

- transport terms $\left(\boldsymbol{u} \cdot \nabla \boldsymbol{u}, \boldsymbol{u} \cdot \nabla \boldsymbol{X}_{t}\right.$ and $\left.\boldsymbol{u} \cdot \nabla \psi\right)$,

- nonlinear terms coming either from the coupling between the equations and $(\boldsymbol{u}, p)$ and $\boldsymbol{\tau}_{p}\left(\nabla \boldsymbol{u} \boldsymbol{X}_{t}\right.$ or $\left.\operatorname{div}_{\boldsymbol{X}}(\nabla \boldsymbol{u} \boldsymbol{X} \psi)\right)$, or inherently contained in the equations defining $\boldsymbol{\tau}_{p}$ (due to the non-linear entropic force $\boldsymbol{F}$ ).

In the next sections, we explain how these difficulties have been addressed both from the mathematical viewpoint and the numerical viewpoint (see also T. Lelièvre [82, and T. Li and P.W. Zhang [85]).

\subsubsection{Simplifications of the equations}

The system (6.7) is quite difficult to study as such. Two simplifications of this general setting are usually considered for preliminary arguments: homogeneous flows and shear flows.

To specifically study the microscopic equations, one can consider homogeneous flows. We recall that in such flows, $\nabla \boldsymbol{u}$ does not depend on the space variable, and therefore $\boldsymbol{X}_{t}$ (and thus $\boldsymbol{\tau}_{p}$ ) does not depend on the space variable either. A solution to (6.7) is then obtained by solving the SDE without the advective term. For a velocity field $\boldsymbol{u}(t, x)=\boldsymbol{\kappa}(t) \boldsymbol{x}, 6.7$ becomes:

$$
\left\{\begin{array}{l}
\boldsymbol{\tau}_{p}=\frac{\epsilon}{\mathrm{We}}\left(\mathbb{E}\left(\boldsymbol{X}_{t} \otimes \boldsymbol{F}\left(\boldsymbol{X}_{t}\right)\right)-\mathrm{Id}\right), \\
d \boldsymbol{X}_{t}=\boldsymbol{\kappa}(t) \boldsymbol{X}_{t} d t-\frac{1}{2 \mathrm{We}} \boldsymbol{F}\left(\boldsymbol{X}_{t}\right) d t+\frac{1}{\sqrt{\mathrm{We}}} d \boldsymbol{W}_{t} .
\end{array}\right.
$$


To keep the difficulty related to the coupling between the macroscopic equation and the microscopic equations but to eliminate the difficulties related to transport terms, many authors (see M. Laso and H.C. Öttinger [75, J.C. Bonvin and M. Picasso [16, C. Guillopé and J.C. Saut [54, B. Jourdain, C. Le Bris and T. Lelièvre 68 or W. E, T. Li and P.W. Zhang [38]) consider shear flows (see Fig. 11. In this geometry, 6.7 writes:

$$
\left\{\begin{array}{l}
\operatorname{Re} \frac{\partial u}{\partial t}(t, y)-(1-\epsilon) \frac{\partial^{2} u}{\partial y^{2}}(t, y)=\frac{\partial \tau}{\partial y}(t, y)+f(t, y) \\
\tau(t, y)=\frac{\epsilon}{\mathrm{We}} \mathbb{E}\left(X_{t}(y) F_{Y}\left(\boldsymbol{X}_{t}(y)\right)\right) \\
d X_{t}(y)=\frac{\partial u}{\partial y}(t, y) Y_{t}(y) d t-\frac{1}{2 \mathrm{We}} F_{X}\left(\boldsymbol{X}_{t}(y)\right) d t+\frac{1}{\sqrt{\mathrm{We}}} d V_{t} \\
d Y_{t}(y)=-\frac{1}{2 \mathrm{We}} F_{Y}\left(\boldsymbol{X}_{t}(y)\right) d t+\frac{1}{\sqrt{\mathrm{We}}} d W_{t}
\end{array}\right.
$$

where $\left(X_{t}(y), Y_{t}(y)\right)$ are the two components of the stochastic process $\boldsymbol{X}_{t}(y),\left(V_{t}, W_{t}\right)$ are two independent Brownian motions and $\left(F_{X}\left(\boldsymbol{X}_{t}\right), F_{Y}\left(\boldsymbol{X}_{t}\right)\right)$ are the two components of the force $\boldsymbol{F}\left(\boldsymbol{X}_{t}\right)$. In this case, $y \in(0,1)$, and Dirichlet boundary conditions are assumed on the velocity at $y=0$ and $y=1$. The initial conditions $\left(X_{0}, Y_{0}\right)$ are assumed to be independent from one another and independent from the Brownian motions.

\subsubsection{Mathematical Analysis}

A fundamental energy estimate In order to understand the mathematical structure of the system (6.7), we first derive an energy estimate. Such an estimate is called an a priori estimate, since it is formally derived assuming sufficient regularity on the solutions for all the manipulations to hold true. These estimates are then used to prove existence and uniqueness results, and, possibly, study longtime properties of the solutions.

Multiplying the momentum equation by $\boldsymbol{u}$ and integrating in space and time, one obtains on the one hand

$$
\begin{aligned}
& \frac{\operatorname{Re}}{2} \int_{\mathcal{D}}|\boldsymbol{u}|^{2}(t, \boldsymbol{x})+(1-\epsilon) \int_{0}^{t} \int_{\mathcal{D}}|\nabla \boldsymbol{u}|^{2}(s, \boldsymbol{x}) \\
& =\frac{\operatorname{Re}}{2} \int_{\mathcal{D}}|\boldsymbol{u}|^{2}(0, \boldsymbol{x})-\frac{\epsilon}{\mathrm{We}} \int_{0}^{t} \int_{\mathcal{D}} \mathbb{E}\left(\boldsymbol{X}_{s}(\boldsymbol{x}) \otimes \boldsymbol{F}\left(\boldsymbol{X}_{s}(\boldsymbol{x})\right)\right): \nabla \boldsymbol{u}(s, \boldsymbol{x}),
\end{aligned}
$$

assuming homogeneous Dirichlet boundary conditions on $\boldsymbol{u}$.

On the other hand, using Itô calculus on $\Pi\left(\boldsymbol{X}_{t}\right)$ (where $\Pi$ is the potential of the force $\boldsymbol{F}$ of the spring), integrating in space, time and taking the expectation value, it is seen that

$$
\begin{array}{rl}
\int_{\mathcal{D}} & \mathbb{E}\left(\Pi\left(\boldsymbol{X}_{t}(\boldsymbol{x})\right)\right)+\frac{1}{2 \mathrm{We}} \int_{0}^{t} \int_{\mathcal{D}} \mathbb{E}\left(\left\|\boldsymbol{F}\left(\boldsymbol{X}_{s}(\boldsymbol{x})\right)\right\|^{2}\right) \\
= & \int_{\mathcal{D}} \mathbb{E}\left(\Pi\left(\boldsymbol{X}_{0}(\boldsymbol{x})\right)\right)+\int_{0}^{t} \int_{\mathcal{D}} \mathbb{E}\left(\boldsymbol{F}\left(\boldsymbol{X}_{s}(\boldsymbol{x})\right) \cdot \nabla \boldsymbol{u}(s, \boldsymbol{x}) \boldsymbol{X}_{s}(\boldsymbol{x})\right) \\
& +\frac{1}{2 \mathrm{We}} \int_{0}^{t} \int_{\mathcal{D}} \Delta \Pi\left(\boldsymbol{X}_{s}(\boldsymbol{x})\right) .
\end{array}
$$


Summing up the two equalities 6.11) and 6.12, and using

$$
\mathbb{E}\left(\boldsymbol{X}_{s}(\boldsymbol{x}) \otimes \boldsymbol{F}\left(\boldsymbol{X}_{s}(\boldsymbol{x})\right)\right): \nabla \boldsymbol{u}(s, \boldsymbol{x})=\mathbb{E}\left(\boldsymbol{F}\left(\boldsymbol{X}_{s}(\boldsymbol{x})\right) \cdot \nabla \boldsymbol{u}(s, \boldsymbol{x}) \boldsymbol{X}_{s}(\boldsymbol{x})\right),
$$

the following energy estimate is obtained:

$$
\begin{aligned}
\frac{\operatorname{Re}}{2} & \frac{d}{d t} \int_{\mathcal{D}}|\boldsymbol{u}|^{2}(t, \boldsymbol{x})+(1-\epsilon) \int_{\mathcal{D}}|\nabla \boldsymbol{u}|^{2}(t, \boldsymbol{x})+\frac{\epsilon}{\mathrm{We}} \frac{d}{d t} \int_{\mathcal{D}} \mathbb{E}\left(\Pi\left(\boldsymbol{X}_{t}(\boldsymbol{x})\right)\right) \\
& +\frac{\epsilon}{2 \mathrm{We}^{2}} \int_{\mathcal{D}} \mathbb{E}\left(\left\|\boldsymbol{F}\left(\boldsymbol{X}_{t}(\boldsymbol{x})\right)\right\|^{2}\right)=\frac{\epsilon}{2 \mathrm{We}^{2}} \int_{\mathcal{D}} \Delta \Pi\left(\boldsymbol{X}_{t}(\boldsymbol{x})\right) .
\end{aligned}
$$

Notice that this energy estimate does not help in the study of the longtime behavior since the term in the right-hand side (which comes form Itô calculus and is non-negative since $\Pi$ is convex) brings energy to the system. We will return to this question below.

As said above, this energy estimate is a first step towards an existence and uniqueness result. For example, in the case of Hookean dumbbells in a shear flow, it allows to prove the following global-in-time existence and uniqueness result (see B. Jourdain, C. Le Bris and T. Lelièvre [67]):

Theorem 2 Assuming $u_{0} \in L_{y}^{2}$ and $f_{\text {ext }} \in L_{t}^{1}\left(L_{y}^{2}\right)$, the system (6.10) for Hookean dumbbells admits a unique solution $(u, X)$ on $(0, T), \forall T>0$. In addition, the following estimate holds:

$$
\begin{aligned}
\|u\|_{L_{t}^{\infty}\left(L_{y}^{2}\right)}^{2} & +\|u\|_{L_{t}^{2}\left(H_{0, y}^{1}\right)}^{2}+\left\|X_{t}\right\|_{L_{t}^{\infty}\left(L_{y}^{2}\left(L_{\omega}^{2}\right)\right)}^{2}+\left\|X_{t}\right\|_{L_{t}^{2}\left(L_{y}^{2}\left(L_{\omega}^{2}\right)\right)}^{2} \\
& \leq C\left(\left\|X_{0}\right\|_{L_{y}^{2}\left(L_{\omega}^{2}\right)}^{2}+\left\|u_{0}\right\|_{L_{y}^{2}}^{2}+T+\left\|f_{e x t}\right\|_{L_{t}^{1}\left(L_{y}^{2}\right)}^{2}\right) .
\end{aligned}
$$

Notice that in this case, $Y_{t}=Y_{0} e^{-t / 2}+\int_{0}^{t} e^{\frac{s-t}{2}} d W_{s}$ is analytically known, so that the existence and uniqueness result only concerns $(u, X)$. The notion of solution employed is: the equation on $u$ is satisfied in the distribution sense and the SDE holds for almost every $(y, \omega)$. The proof relies on a variational formulation of the PDE, and follows a very classical line. It consists in (i) building a sequence of approximate solutions (by a Galerkin procedure), (ii) using the energy estimate (which indeed has then a rigorous, better than formal, meaning) to derive some bounds on this sequence from which one deduces the existence of a limit (up to the extraction of a subsequence), (iii) passing to the limit in the variational formulation of the PDE. This approach is interesting since, as is well known, it is also useful to prove the convergence of numerical methods based on variational formulations (such as finite element methods).

This setting (Hookean dumbbell in a shear flow) is actually extremely specific. A globalin-time existence and uniqueness result is obtained since the coupling term $\nabla \boldsymbol{u} \boldsymbol{X}_{t}$ of the original problem (6.7) simplifies to $\frac{\partial u}{\partial y} Y_{t}$ in (6.10), where $Y_{t}$ is known independently of $(u, X)$. In other words, this coupling term is no more nonlinear.

For FENE dumbbell, two new difficulties have to be addressed: first, the SDE contains an explosive drift term and second, even in a shear flow, the coupling term $\nabla \boldsymbol{u} \boldsymbol{X}_{t}$ is genuinely nonlinear. 
The FENE SDE In this paragraph, we consider the FENE SDE in a given homogeneous flow. As we mentioned earlier, the FENE force has been introduced to prevent the length of the dumbbell from exceeding the maximal length of the polymer. What can be actually proven is the following (see B. Jourdain and T. Lelièvre [66]):

Proposition 1 Let us consider the SDE in 6.9 for FENE force: $\boldsymbol{F}(\boldsymbol{X})=\frac{\boldsymbol{X}}{1-\|\boldsymbol{X}\|^{2} / b}$.

- For $\kappa \in L_{\mathrm{loc}}^{1}\left(\mathbb{R}_{+}\right)$and $b>0$, this $S D E$ admits a strong solution with values in $B=$ $\mathcal{B}(0, \sqrt{b})$, which is unique in the class of solutions with values in $B=\mathcal{B}(0, \sqrt{b})$.

- Assume $\kappa \in L^{2}\left(\mathbb{R}_{+}\right)$. If $b \geq 2$, then the solution does not touch the boundary of $B$ in finite time. If $0<b<2$, The solution touches (a.s.) the boundary of $B$ in finite time.

- Take $\kappa \equiv 0$ (for simplicity) and $0<b<2$. It is possible to build two different stochastic processes satisfying the SDE.

In practice, $b$ is typically larger than 10 , so that the SDE has indeed a unique strong solution.

The FENE model in a Couette flow As mentioned above, for the FENE model in the Couette flow, the coupling term $\frac{\partial u}{\partial y} Y_{t}$ is indeed nonlinear since $Y_{t}$ depends on $X_{t}$ (through the force term $\left.F_{Y}\left(\boldsymbol{X}_{t}\right)\right)$ and thus on $u$. This nonlinearity implies additional difficulties in the existence result, and the a priori estimate we derived above does not provide enough regularity on the velocity to pass to the limit in the nonlinear term $\frac{\partial u}{\partial y}(t, y) Y_{t}$.

The question is then: for a given regularity of $u$ (say $u \in L_{t}^{\infty}\left(L_{y}^{2}\right) \cap L_{t}^{2}\left(H_{0, y}^{1}\right)$ if we consider the first energy estimate), what is the regularity of $\tau$ ? Formally, owing to the presence of the nonlinear term $\nabla \boldsymbol{u} \boldsymbol{X}_{t}$ in the SDE, $\tau$ has the regularity of $\exp \left(\int_{0}^{t} \frac{\partial u}{\partial y}\right)$ which may be very irregular if one only assumes $u \in \in L_{t}^{\infty}\left(L_{y}^{2}\right) \cap L_{t}^{2}\left(H_{0, y}^{1}\right)$.

One way to address this difficulty is to derive additional a priori regularity on the velocity. This can be performed by multiplying the equation on $u$ in (6.10) by $-\frac{\partial^{2} u}{\partial y^{2}}$ and using Girsanov theorem to explicitly obtain the dependency of $\tau$ in terms of $u$ :

$$
\begin{aligned}
\tau(t, y) & =\mathbb{E}\left(\frac{X_{t}(y) Y_{t}(y)}{1-\frac{\left(X_{t}(y)\right)^{2}+\left(Y_{t}(y)\right)^{2}}{b}}\right) \\
& =\mathbb{E}\left(\left(\frac{\tilde{X}_{t} \tilde{Y}_{t}}{1-\frac{\tilde{X}_{t}^{2}+\tilde{Y}_{t}^{2}}{b}}\right) \mathcal{E}\left(\frac{1}{\sqrt{\mathrm{We}}} \int_{0}^{\bullet} \frac{\partial u}{\partial y}(y) \tilde{Y}_{s} d V_{s}\right)_{T}\right)
\end{aligned}
$$

where $\widetilde{\boldsymbol{X}}_{t}=\left(\tilde{X}_{t}, \tilde{Y}_{t}\right)$ is the stochastic process satisfying the FENE SDE with $\frac{\partial u}{\partial y}=0$ :

$$
d \widetilde{\boldsymbol{X}}_{t}=-\frac{1}{2 \mathrm{We}} \frac{\widetilde{\boldsymbol{X}}_{t}}{1-\left\|\widetilde{\boldsymbol{X}}_{t}\right\|^{2} / b} d t+\frac{1}{\sqrt{\mathrm{We}}} d \boldsymbol{W}_{t},
$$


and $\mathcal{E}$ is the exponential martingale:

$$
\mathcal{E}\left(\frac{1}{\sqrt{\mathrm{We}}} \int_{0}^{\bullet} \frac{\partial u}{\partial y} \tilde{Y}_{s} d V_{s}\right)_{t}=\exp \left(\frac{1}{\sqrt{\mathrm{We}}} \int_{0}^{t} \frac{\partial u}{\partial y} \tilde{Y}_{s} d V_{s}-\frac{1}{2 \mathrm{We}} \int_{0}^{t}\left(\frac{\partial u}{\partial y} \tilde{Y}_{s}\right)^{2} d s\right) .
$$

Owing to the exponential dependency of $\tau$ on $u$ in (6.15), this additional a priori estimate yields bounds on $u$ in $L_{t}^{\infty}\left(H_{0, y}^{1}\right) \cap L_{t}^{2}\left(H_{y}^{2}\right)$-norm but only locally in time.

The following local-in-time existence and uniqueness result can then be proven (see B. Jourdain, C. Le Bris and T. Lelièvre [68]):

Theorem 3 Under the assumptions $b>6, f_{\text {ext }} \in L_{t}^{2}\left(L_{y}^{2}\right)$ and $u_{0} \in H_{y}^{1}, \exists T>0$ (depending on the data) s.t. the system admits a unique solution $(u, X, Y)$ on $[0, T)$. This solution is such that $u \in L_{t}^{\infty}\left(H_{0, y}^{1}\right) \cap L_{t}^{2}\left(H_{y}^{2}\right)$. In addition, we have:

- $\mathbb{P}\left(\exists t>0,\left(\left(X_{t}^{y}\right)^{2}+\left(Y_{t}^{y}\right)^{2}\right)=b\right)=0$,

- $\left(X_{t}^{y}, Y_{t}^{y}\right)$ is adapted with respect to the filtration $\mathcal{F}_{t}^{V, W}$ associated with the Brownian motions.

For a similar result in a more general setting (3-dimensional flow) and forces with polynomial growth, we refer to W. E, T. Li and P.W. Zhang [39. The authors prove a localin-time existence and uniqueness result in high Sobolev spaces. We also refer to A. Bonito, $\mathrm{Ph}$. Clément and M. Picasso [15] for existence results for Hookean dumbbells, neglecting the advection terms. When the velocity field is not regular enough, it is difficult to give a sense to the transport term in the SDE (which is actually a Stochastic Partial Differential Equation). We refer to C. Le Bris and P.-L. Lions [78, 79].

Longtime behavior As we mentioned above, the a priori estimate 6.14 cannot be used to understand the longtime behavior of the system because of the non-negative term $\frac{\epsilon}{2 \mathrm{We}^{2}} \int_{\mathcal{D}} \Delta \Pi\left(\boldsymbol{X}_{t}(\boldsymbol{x})\right)$ in the right-hand side. It actually appears that eliminating this term requires to add an entropy term to the energy. To study the longtime behavior, the appropriate viewpoint is to consider the free energy rather than the energy.

To introduce the entropy, one needs to consider the probability density functional of the stochastic process $\boldsymbol{X}_{t}$, and thus the system (6.8) coupling the momentum equation with the Fokker-Planck equation introduced in Section 3.2. Let us assume zero Dirichlet boundary condition on the velocity $\boldsymbol{u}$. The expected stationary state (equilibrium) is

$$
\begin{aligned}
\boldsymbol{u}(\infty, \boldsymbol{x}) & =0, \\
\psi(\infty, \boldsymbol{x}, \boldsymbol{X})=\psi_{\mathrm{eq}}(\boldsymbol{X}) & =C \exp (-\Pi(\boldsymbol{X})),
\end{aligned}
$$

where $C$ is a normalization factor. Using entropy estimates (see C. Ané et al. [4, F. Malrieu [94, A. Arnold, P. Markowich, G. Toscani and A. Unterreiter [5]), exponential convergence to equilibrium may be shown (see B. Jourdain, C. Le Bris, T. Lelièvre and F. Otto 64, 65]). Let us explain this with more details. 
The first derivative of the kinetic energy

$$
E(t)=\frac{\operatorname{Re}}{2} \int_{\mathcal{D}}|\boldsymbol{u}|^{2}(t, \boldsymbol{x})
$$

writes (as in 6.11)

$$
\frac{d E}{d t}=-(1-\epsilon) \int_{\mathcal{D}}|\nabla \boldsymbol{u}|^{2}(t, \boldsymbol{x})-\frac{\epsilon}{\mathrm{We}} \int_{\mathcal{D}} \int_{\mathbb{R}^{d}}(\boldsymbol{X} \otimes \nabla \Pi(\boldsymbol{X})): \nabla \boldsymbol{u}(t, \boldsymbol{x}) \psi(t, \boldsymbol{x}, \boldsymbol{X}) .
$$

The entropy

$$
\begin{aligned}
H(t) & =\int_{\mathcal{D}} \int_{\mathbb{R}^{d}} \Pi(\boldsymbol{X}) \psi(t, \boldsymbol{x}, \boldsymbol{X})+\int_{\mathcal{D}} \int_{\mathbb{R}^{d}} \psi(t, \boldsymbol{x}, \boldsymbol{X}) \ln (\psi(t, \boldsymbol{x}, \boldsymbol{X}))-|\mathcal{D}| \ln C, \\
& =\int_{\mathcal{D}} \int_{\mathbb{R}^{d}} \psi(t, \boldsymbol{x}, \boldsymbol{X}) \ln \left(\frac{\psi(t, \boldsymbol{x}, \boldsymbol{X})}{\psi_{\mathrm{eq}}(\boldsymbol{X})}\right)
\end{aligned}
$$

is next introduced. Notice that $H(t) \geq 0($ since $x \ln (x) \geq x-1)$. Using (6.13) and $\operatorname{div} \boldsymbol{u}=0$, a simple computation shows:

$$
\begin{aligned}
\frac{d H}{d t}= & -\frac{1}{2 \mathrm{We}} \int_{\mathcal{D}} \int_{\mathbb{R}^{d}} \psi(t, \boldsymbol{x}, \boldsymbol{X})\left|\nabla_{\boldsymbol{X}} \ln \left(\frac{\psi(t, \boldsymbol{x}, \boldsymbol{X})}{\psi_{\mathrm{eq}}(\boldsymbol{X})}\right)\right|^{2} \\
& +\int_{\mathcal{D}} \int_{\mathbb{R}^{d}}(\boldsymbol{X} \otimes \nabla \Pi(\boldsymbol{X})): \nabla \boldsymbol{u}(t, \boldsymbol{x}) \psi(t, \boldsymbol{x}, \boldsymbol{X}) .
\end{aligned}
$$

Thus, the free energy $F(t)=E(t)+\frac{\epsilon}{\mathrm{We}} H(t)$ (a non-negative quantity) satisfies:

$$
\frac{d F}{d t}=-(1-\epsilon) \int_{\mathcal{D}}|\nabla \boldsymbol{u}|^{2}(t, \boldsymbol{x})-\frac{\epsilon}{2 \mathrm{We}^{2}} \int_{\mathcal{D}} \int_{\mathbb{R}^{d}} \psi(t, \boldsymbol{x}, \boldsymbol{X})\left|\nabla \boldsymbol{X} \ln \left(\frac{\psi(t, \boldsymbol{x}, \boldsymbol{X})}{\psi_{\mathrm{eq}}(\boldsymbol{X})}\right)\right|^{2} .
$$

Comparing with (6.14), we observe that the introduction of the entropy allows to eliminate the right-hand side. In particular, (6.18) shows that the only stationary state is $\boldsymbol{u}=0$ et $\psi=\psi_{\text {eq. }}$. Moreover, using a Poincaré inequality: for all $\boldsymbol{u} \in H_{0}^{1}(\mathcal{D})$,

$$
\int|\boldsymbol{u}|^{2} \leq C \int|\nabla \boldsymbol{u}|^{2}
$$

and the Logarithmic Sobolev inequality: for all probability density functional $\psi$,

$$
\int \psi \ln \left(\frac{\psi}{\psi_{\text {eq }}}\right) \leq C \int \psi\left|\nabla \ln \left(\frac{\psi}{\psi_{\text {eq }}}\right)\right|^{2}
$$

exponential convergence to zero for $F$ (and thus for $\boldsymbol{u}$ in $L_{\boldsymbol{x}}^{2}$-norm) is obtained from (6.18). The Logarithmic Sobolev inequality (6.19) holds for $\psi_{\mathrm{eq}}(\boldsymbol{X})=C \exp (-\Pi(\boldsymbol{X}))$ if $\Pi$ is $\alpha$ convex for example (which is the case for Hookean and FENE dumbbells). The CsiszarKullback inequality (see C. Ané et al. [4]) then shows that $\psi$ converges to $\psi_{\text {eq }}$ exponentially fast in $L_{\boldsymbol{x}}^{2}\left(L_{\boldsymbol{X}}^{1}\right)$-norm. 
For generalizations of these computations to non-homogeneous boundary conditions on $\boldsymbol{u}$ (and thus $\boldsymbol{u}(\infty, \boldsymbol{x}) \neq 0$ ), we refer to B. Jourdain, C. Le Bris, T. Lelièvre and F. Otto [65].

We would like also to mention that these estimates on the micro-macro system can be used as a guideline to derive new estimates on related macro-macro models (see D. Hu and T. Lelièvre [59]).

Remark 6.1 (On the choice of the entropy) If one considers the Fokker-Planck equation with $\boldsymbol{u}=0$, it is well-known (see A. Arnold, P. Markowich, G. Toscani and A. Unterreiter [5]) that exponential convergence to equilibrium can be obtained using more general entropy functions of the form

$$
H(t)=\int_{\mathcal{D}} \int_{\mathbb{R}^{d}} h\left(\frac{\psi}{\psi_{\mathrm{eq}}}\right) \psi_{\mathrm{eq}}
$$

where $h: \mathbb{R} \rightarrow \mathbb{R}_{+}^{*}$ is a convex $\mathcal{C}^{2}$ function, such that $h(1)=0$. However, it seems that to derive the entropy estimate [6.18 on the coupled system [6.10), it is necessary to choose the "physical entropy" corresponding to the choice $h(x)=x \ln (x)-(x-1)$.

Remark 6.2 (On the assumptions on the force $\boldsymbol{F}$ ) Recall that we assumed that $\boldsymbol{F}=$ $\nabla \Pi$, where $\Pi$ is a radial convex function. Let us briefly discuss the assumptions on $\boldsymbol{F}$ we used so far.

- The fact that $\boldsymbol{F}$ can be written as the gradient of a potential $\Pi$ is important to obtain a simple analytical expression for $\psi_{\mathrm{eq}}$.

- The fact that $\Pi$ is radial is a very important assumption to ensure the symmetry of the stress tensor.

- The convexity assumption on $\Pi$ is important in the analysis of the SDEs (in particular for uniqueness of strong solutions).

- The $\alpha$-convexity of the potential $\Pi$ has been used to obtain the Logarithmic Sobolev inequality (6.19).

Existence results on the coupled problem with the Fokker-Planck PDE Many authors have obtained existence and uniqueness results for the micro-macro problem in its coupled form (6.8) with the Fokker-Planck equation.

For local existence and uniqueness results, we refer to M. Renardy [110], T. Li, H. Zhang and P.W. Zhang [83] (polynomial forces) and to H. Zhang and P.W. Zhang 122] (FENE force with $b>76$ ). In a recent work by N. Masmoudi [96, a local in time existence result is obtained for the FENE model without any assumption on $b$ (using reflecting boundary conditions). The author also shows global in time existence result for initial data close to equilibrium (see also F. Lin, C. Liu and P.W. Zhang [87] for a similar result under the assumption $b>12)$.

Global existence results have also been obtained for closely related problems: 
- In J.W. Barrett, C. Schwab and E. Süli [7, 8, a global existence result is obtained for (6.8) (Hookean and FENE force) using a regularization of some differential operators present in these equations, so as to bootstrap more regularity on the solution. Se also L. Zhang, H. Zhang and P.W. Zhang [123].

- In J.W. Barrett, C. Schwab and E. Süli [7, 8] (again with some regularizations) and P.-L. Lions and N. Masmoudi [89, 96] (without any regularizations), the authors obtain global-in-time existence results by changing $\nabla \boldsymbol{u}$ in the Fokker-Planck equation to $\frac{\nabla \boldsymbol{u}-\nabla \boldsymbol{u}^{T}}{2}$ (which is similar to considering the corotational derivative rather than the upper convected derivative on the PDE on $\tau_{p}$ in macro-macro models).

We would like also to mention the following related works (existence results for coupled Navier-Stokes Fokker-Planck micro-macro models) by P. Constantin, C. Fefferman, N. Masmoudi and E.S. Titi: [27, 28, 31], and also the work of C. Le Bris and P.-L. Lions [78, 79] about existence and uniqueness of solutions to Fokker-Planck type equations with irregular coefficients.

\subsubsection{Numerical methods}

In this section, we review the literature for the numerical analysis of methods to discretize (4.46). For the discretization of the micro-macro problem in the Fokker-Planck version, we refer to Section 4.4

The idea of coupling a Finite Element Method for discretization in space and a stochastic method (Monte Carlo to approximate the expectation and Euler scheme on the SDE) has been first proposed by M. Laso and H.C. Öttinger [75]. Such a method is called Calculation Of Non-Newtonian Flow: Finite Elements and Stochastic SImulation Technique (CONNFFESSIT). At first, Lagrangian methods were used on the SDE, and independent Brownian motions on each trajectories (see M. Laso and H.C. Öttinger [76]). The algorithm then consists in: (i) computing $(\boldsymbol{u}, p)$, (ii) computing the trajectories of the fluid particles carrying the dumbbells (characteristic method), (iii) integrating the SDEs along these trajectories and (iv) computing the stress tensor $\tau_{p}$ by local empirical means in each finite element. This Lagrangian approach is the most natural one since it is naturally obtained from the derivation of the model (see Section 4.2.1). However, owing to the term $\operatorname{div} \tau_{p}$, numerical results are very noisy in space when using independent Brownian motions on each trajectory. Moreover, such an approach requires to maintain a sufficiently large number of dumbbells per cell of of the mesh, which is not easy to satisfy (there is a need to add some dumbbells and to destroy others during the simulation).

The idea then came up to use the Eulerian version of the SDE, and introducing fields of end-to-end vectors: $\boldsymbol{X}_{t}(\boldsymbol{x})$. This is the concept of Brownian Configuration Field introduced by M.A. Hulsen, A.P.G. van Heel and B.H.A.A. van den Brule in 61. In this Eulerian description, the most natural and simple choice is to use the same Brownian motion at each position in space. This reduces the noise in space and the variance on the velocity (but not the variance on the stress, see below and the work [63] by B. Jourdain, C. Le Bris and T. Lelièvre). The discretization of the transport term can then be done using a Discontinuous 
Galerkin methods (see M.A. Hulsen, A.P.G. van Heel and B.H.A.A. van den Brule [61]), the characteristic methods (see J.C. Bonvin [18] or the Backward-Tracking Lagrangian Particle Method of P. Wapperom, R. Keunings and V. Legat [120]), or classical finite element methods with stabilization.

Let us recall how the CONNFFESSIT method writes in a shear flow (see Section [5.4). In this special case, both the Lagrangian and the Eulerian approaches lead to the same discretization: for given $u_{h}^{n}, X_{h, n}^{k}$ and $Y_{h, n}^{k}$, compute $u_{h}^{n+1} \in V_{h}$ such that for all $v \in V_{h}$,

$$
\left\{\begin{aligned}
& \frac{\operatorname{Re}}{\delta t} \int_{y}\left(u_{h}^{n+1}-u_{h}^{n}\right) v=-(1-\epsilon) \int_{y} \frac{\partial u_{h}^{n+1}}{\partial y} \frac{\partial v}{\partial y}-\int_{y} \tau_{h, n} \frac{\partial v}{\partial y}+\int_{y} f v \\
& \tau_{h, n}=\frac{\epsilon}{\mathrm{We}} \frac{1}{K} \sum_{k=1}^{K} X_{h, n}^{k} F_{Y}\left(X_{h, n}^{k}, Y_{h, n}^{k}\right) \\
& X_{h, n+1}^{k}-X_{h, n}^{k}=\left(\frac{\partial u_{h}^{n+1}}{\partial y} Y_{h, n}^{k}-\frac{1}{2 \mathrm{We}} F_{X}\left(X_{h, n}^{k}, Y_{h, n}^{k}\right)\right) \delta t \\
&+\frac{1}{\sqrt{\mathrm{We}}}\left(V_{h, t_{n+1}}^{k}-V_{h, t_{n}}^{k}\right) \\
& Y_{h, n+1}^{k}-Y_{h, n}^{k}=-\frac{1}{2 \mathrm{We}} F_{Y}\left(X_{h, n}^{k}, Y_{h, n}^{k}\right) \delta t+\frac{1}{\sqrt{\mathrm{We}}}\left(W_{h, t_{n+1}}^{k}-W_{h, t_{n}}^{k}\right) .
\end{aligned}\right.
$$

The index $n$ is the timestep and the index $k$ is the realization number in the $\operatorname{SDE}(1 \leq k \leq K$ where $K$ is the number of dumbbells in each cell). Finally, $V_{h}$ is a finite element space. We suppose in the following that $V_{h}=\mathrm{P} 1$ is the finite element space of continuous piecewise linear functions so that $X_{h, n}, Y_{h, n}$ and $\tau_{h, n}$ are piecewise constant functions in space (they belong to the functional space P0). We refer to Figure 13.

Convergence of the CONNFFESSIT method In the CONNFFESSIT method, three numerical parameters are to be chosen: the timestep $\delta t$, the spacestep $h$ and the number of dumbbells (or realizations) $K$. It is expected that the method converges in the limit $\delta t \rightarrow 0$, $h \rightarrow 0$ and $K \rightarrow \infty$.

This has been proven in B. Jourdain, C. Le Bris and T. Lelièvre [67] and W. E, T. Li and P.W. Zhang [38] for Hookean dumbbells in a shear flow.

Theorem 4 Assuming $u_{0} \in H_{y}^{2}, f_{\text {ext }} \in L_{t}^{1}\left(H_{y}^{1}\right)$, $\frac{\partial f_{\text {ext }}}{\partial t} \in L_{t}^{1}\left(L_{y}^{2}\right)$ and $\delta t<\frac{1}{2}$, we have (for $\left.V_{h}=\mathrm{P} 1\right): \forall n<\frac{T}{\delta t}$,

$$
\begin{aligned}
\left\|u\left(t_{n}\right)-\bar{u}_{h}^{n}\right\|_{L_{y}^{2}\left(L_{\omega}^{2}\right)}+\| \mathbb{E}\left(X_{t_{n}} Y_{t_{n}}\right)- & \frac{1}{K} \sum_{k=1}^{K} \bar{X}_{h, n}^{k} \bar{Y}_{n}^{k} \|_{L_{y}^{1}\left(L_{\omega}^{1}\right)} \\
& \leq C\left(h+\delta t+\frac{1}{\sqrt{K}}\right) .
\end{aligned}
$$

Remark 6.3 It can be shown that the convergence in space is optimal (see T. Lelièvre [81]):

$$
\left\|u\left(t_{n}\right)-\bar{u}_{h}^{n}\right\|_{L_{y}^{2}\left(L_{\omega}^{2}\right)} \leq C\left(h^{2}+\delta t+\frac{1}{\sqrt{K}}\right) .
$$




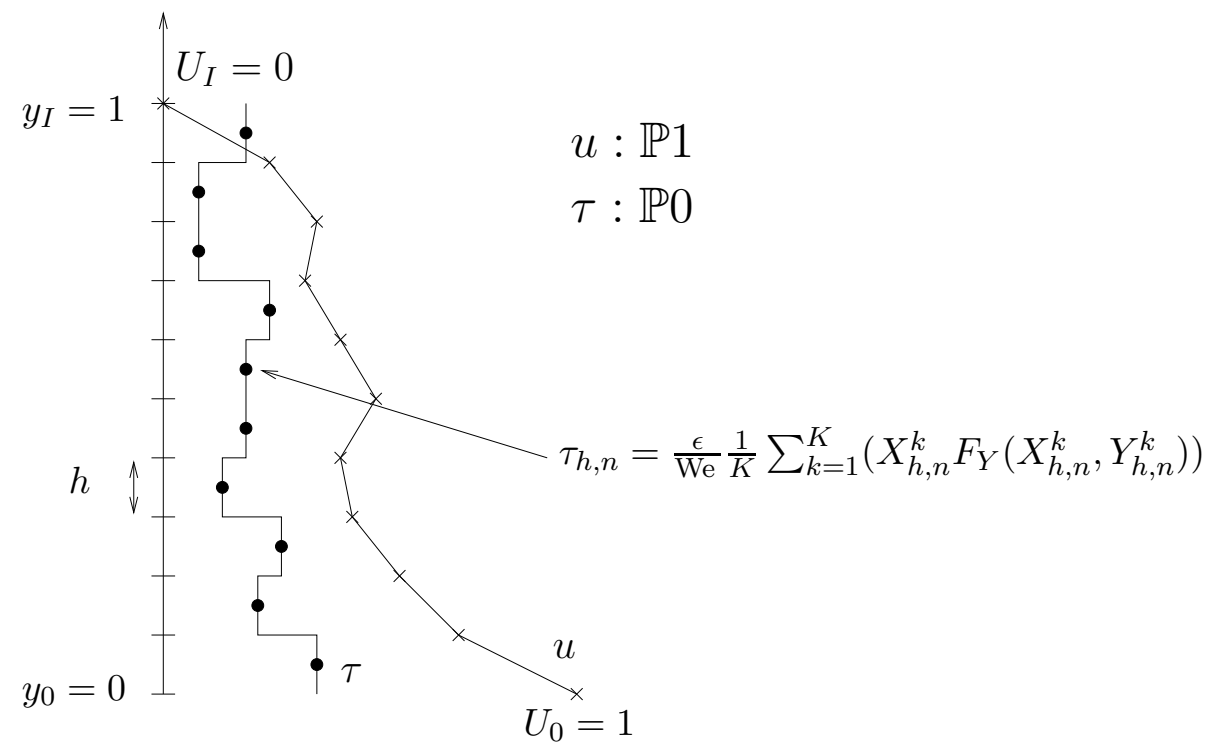

Figure 13: The CONNFFESSIT method in a shear flow.

The main difficulties in the proof of Theorem 4 originate from the following facts:

- The velocity $u_{h}^{n}$ is a random variable. The energy estimate at the continuous level cannot be directly translated into an energy estimate at the discrete level (which would yield the stability of the scheme).

- The end-to-end vectors $\left(\bar{X}_{h, n}^{k}, \bar{Y}_{n}^{k}\right)_{1 \leq k \leq K}$ are coupled random variables (even though the driving Brownian motions $\left(V_{h, t}^{k}, W_{h, t}^{k}\right)_{1 \leq k \leq K}$ are independent).

- The stability of the numerical scheme requires an almost sure bound on the $Y_{n}^{k}$ :

$$
\delta t \frac{1}{K} \sum_{k=1}^{K}\left(Y_{n}^{k}\right)^{2}<1 .
$$

To prove convergence, a cut-off procedure on $Y_{n}^{k}$ is employed:

$$
\bar{Y}_{n+1}^{k}=\max \left(-A, \min \left(A, Y_{n+1}^{k}\right)\right)
$$

with $0<A<\sqrt{\frac{3}{5 \delta t}}$. In Theorem $4, \bar{u}_{h}^{n}, \bar{X}_{n}^{k} \bar{Y}_{n}^{k}$ denotes random variables obtained by the CONNFFESSIT scheme [6.20) with the cutoff procedure 6.21). It can be checked that for sufficiently small $\delta t$ or sufficiently large $K$, this cut-off procedure is not used. 
For a result without cut-off, we refer to B. Jourdain, C. Le Bris and T. Lelièvre [67. For an extension of these results to a more general geometry and discretization by a finite difference scheme, we refer to T. Li and P.W. Zhang [84]. For a convergence results in space and time, we refer to A. Bonito, Ph. Clément and M. Picasso [14.

Variance of the results and dependency of the Brownian motions in space One important practical quantity when using Monte Carlo methods is the variance of the result. If the variance is too large, the numerical method is basically useless. We already mentioned above (see Section 5.5) variance reduction methods. It is also interesting to investigate how the variance of the results depends upon the numerical parameters. In the framework of the CONNFFESSIT method, this variance is particularly sensitive to the dependency of the Brownian motion on the space variable.

One can check (at least for regular solutions) that the dependency of the Brownian motion on the space variable does not influence the macroscopic quantities $\left(\boldsymbol{u}, p, \boldsymbol{\tau}_{p}\right)$ at the continuous level. This can be rigorously proved for Hookean dumbbells in a shear flow. It can also be checked that the convergence result of Theorem 4 is insensitive to the dependency of the Brownian motion on the space variable. However, at the discrete level, this dependency strongly influences the variance of the results. It is observed that using Brownian motions independent from one cell of the mesh to another rather than Brownian motions not depending on space increases the variance of the velocity, but reduces the variance on the stress (see P. Halin, G. Lielens, R. Keunings, and V. Legat [57, J.C. Bonvin and M. Picasso [16] and B. Jourdain, C. Le Bris and T. Lelièvre [63]).

This can be precisely analyzed for the case of Hookean dumbbells in a shear flow. It can be shown that (see B. Jourdain, C. Le Bris and T. Lelièvre [63]):

a) The variance on the velocity is minimum for a Brownian motion not depending on space.

b) Using Brownian motions independent from one cell to another is not the best method to reduce the variance on $\tau$.

c) It is possible to reduce the variance on $\tau$ with the same computational cost as when using a Brownian motion not depending on space. It consists in using a Brownian motion alternatively multiplied by +1 or -1 on nearest-neighbour cells.

\section{Other types of complex fluids}

\subsection{Liquid crystals}

So far, we have only considered dilute solutions of flexible polymers. Some other polymers behave more like rigid rods. This introduces anisotropy in the system. Solutions of such rigid polymers are called polymeric liquid crystals. One of the major aspect to account for 
in the modelling of solutions of rod-like polymers is that the interaction of the polymers becomes important at much a lower concentration than with flexible polymers.

Modelling of liquid crystals, along with mathematical and numerical studies, is today a very lively and active field of research. The present short section does not reflect the variety of scientific enterprises dealing with liquid crystals. It is just a brief incursion in this world to see, once, the basic models. One adequate model is the Doi model (see M. Doi and S.F. Edwards [36] and H.C. Öttinger [101]). It describes the evolution for a configuration vector $\boldsymbol{R}_{t}$ by a stochastic differential equation:

$$
\begin{aligned}
d \boldsymbol{R}_{t}+ & \boldsymbol{u} \cdot \nabla \boldsymbol{R}_{t} d t \\
= & \left(\mathrm{Id}-\frac{\boldsymbol{R}_{t} \otimes \boldsymbol{R}_{t}}{\left\|\boldsymbol{R}_{t}\right\|^{2}}\right)\left(\left(\nabla \boldsymbol{u} \boldsymbol{R}_{t}-\frac{1}{2} B^{2} \nabla V\left(\boldsymbol{R}_{t}\right)\right) d t+B d \boldsymbol{W}_{t}\right) \\
& -\frac{d-1}{2} B^{2} \frac{\boldsymbol{R}_{t}}{\left\|\boldsymbol{R}_{t}\right\|^{2}} d t,
\end{aligned}
$$

where $B$ is a positive constant and $d=2$ or 3 is the dimension of the ambient space. Notice that $B$ may also be a function $B\left(\boldsymbol{R}_{t}\right)$ in some models (with then an additional term involving $\nabla\left(B^{2}\right)$ in the drift term). Notice also that we assume that all the initial conditions $\boldsymbol{R}_{0}(\boldsymbol{x})$ have a fixed length $L$ so that $\forall(t, \boldsymbol{x}),\left\|\boldsymbol{R}_{t}(\boldsymbol{x})\right\|=\left\|\boldsymbol{R}_{0}(\boldsymbol{x})\right\|=L$. The potential $V$ accounts for the mean-field interaction between the polymers. For example, the Maier-Saupe potential is:

$$
V(\boldsymbol{R})=-\frac{1}{L^{4}} \mathbb{E}\left(\boldsymbol{R}_{t} \otimes \boldsymbol{R}_{t}\right): \boldsymbol{R} \otimes \boldsymbol{R} .
$$

The stress tensor is then given by:

$$
\boldsymbol{\tau}_{p}(t)=\mathbb{E}\left(\boldsymbol{u}_{t} \otimes \boldsymbol{u}_{t}\right)+\mathbb{E}\left(\boldsymbol{u}_{t} \otimes\left(\left(\mathrm{Id}-\boldsymbol{u}_{t} \otimes \boldsymbol{u}_{t}\right) \nabla V\left(\boldsymbol{u}_{t}\right)\right)\right)-\mathrm{Id}
$$

where $\boldsymbol{u}_{t}=\frac{\boldsymbol{R}_{t}}{L}$ is the rod orientation. We have neglected the viscous contribution in (7.3). The fully coupled system then consists in the first two equations of (3.13) with (7.1)-(7.3). Notice that the main differences with the equations seen so far in this article are the nonlinearity in the sense of MacKean due to the presence of the expectation value in the potential $V$ and the fact that the diffusion term depends on the process $\boldsymbol{R}_{t}$.

For an analysis of the coupled system with the Fokker-Planck version of (7.1)-(7.3) in the special case of shear flow, we refer to H. Zhang and P.W. Zhang [121]. The longtime behavior of the Fokker-Planck equation has been studied by P. Constantin, I. Kevrekidis and E.S. Titi in [30 (see also [29]). A thorough analysis of the variety of possible steady states and their stability is studied by G. Forest, Q. Wang and R. Zhou in [45]. Some numerical methods to solve the stochastic differential equation (7.1) are proposed by H.C. Öttinger in [101]. On the other hand, we are not aware of any rigorous numerical analysis of numerical methods to solve this system without closure approximation. 


\subsection{Suspensions}

We now slightly change the context. Multiscale modelling of complex fluids is very advanced for polymer flows. It is a well established scientific activity. However, it is also a growing activity for some other types of fluids, far from polymer flows. We give here the illustrative example of civil engineering fluids, with muds and clays. It is not forbidden to believe that other materials of civil engineering, like cement, will benefit a lot from multiscale modelling approaches in a near future.

For concentrated suspensions (such as muds or clays), one model available in the literature is the Hebraud-Lequeux model [62]. This model describes the rheology of the fluid in terms of a Fokker-Planck equation ruling the evolution in time of the probability of finding, at each point, the fluid in a given state of stress. To date, although current research is directed toward constructing multidimensional variants, the model is restricted to the onedimensional setting, that is, the Couette flow. The stress at the point $y$ and at time $t$ is thus determined by one scalar variable $\sigma$ :

$$
\left\{\begin{aligned}
\frac{\partial p}{\partial t}(t, y, \sigma) & =-\frac{\partial u}{\partial y}(t, y) \frac{\partial p}{\partial \sigma}(t, y, \sigma)+D(p) \frac{\partial^{2} p}{\partial \sigma^{2}}(t, y, \sigma) \\
& -H(|\sigma|-1) p(t, y, \sigma)+D(p) \delta_{0}, \\
D(p) & =\int_{|\sigma| \geq 1} p(t, y, \sigma) d \sigma .
\end{aligned}\right.
$$

In the above system, where we have on purpose omitted all physical constants, the function $H$ denotes the Heaviside function. It aims at modelling the presence of a threshold constraint (here set to one): when the constraint is above the threshold, the stress relaxes to zero, which translates into the two last terms of the Fokker-Planck equation. The diffusion in the stress space is also influenced nonlinearly by the complete state of stress, as indicated by the definition of $D(p)$. On the other hand, the function $\frac{\partial u}{\partial y}(t, y)$ accounts for a shear rate term, here provided by the macroscopic flow. The contribution to the stress at the point $y$ under consideration is then given by the average

$$
\tau(t, y)=\int_{\mathbb{R}} \sigma p(t, y, \sigma) d \sigma .
$$

The fully coupled system consisting of the Fokker-Planck equation (7.4), the expression (7.5) of the stress tensor, and the macroscopic equation for the Couette flow (first line of (5.6)) has been studied mathematically in a series of work by E. Cancès, I. Catto, Y. Gati and C. Le Bris [21, 22, 23].

Alternately to a direct attack of the Fokker-Planck equation (7.4), one might wish to simulate the associated stochastic differential equation with jumps that reads

$$
d \sigma_{t}=\frac{\partial u}{\partial y} d t+\sqrt{2 \mathbb{P}\left(\left|\sigma_{t}\right| \geq 1\right)} d W_{t}-1_{\left\{\left|\sigma_{t^{-}}\right| \geq 1\right\}} \sigma_{t^{-}} d N_{t}
$$


where $W_{t}$ is a Brownian motion and $N_{t}$ is an independent Poisson process with unit intensity. Note that, in addition to the jumps, equation (17.6) is nonlinear in the sense of MacKean, as the diffusion coefficient depends on the marginal law of the solution at time $t$.

The coupled system to simulate then reads

$$
\left\{\begin{array}{l}
\frac{\partial u}{\partial t}(t, u)-\frac{\partial^{2} u}{\partial y^{2}}(t, y)=\frac{\partial \tau}{\partial y}(t, y) \\
\forall y,\left\{\begin{array}{l}
\tau(t, y)=\mathbb{E}\left(\sigma_{t}(y)\right) \\
d \sigma_{t}(y)=\frac{\partial u}{\partial y} d t+\sqrt{2 \mathbb{P}\left(\left|\sigma_{t}(y)\right| \geq 1\right)} d W_{t}-1_{\left\{\left|\sigma_{t^{-}}(y)\right| \geq 1\right\}} \sigma_{t^{-}}(y) d N_{t}
\end{array}\right.
\end{array}\right.
$$

where one should note that the stochastic differential equation has jumps.

Numerical simulations of this system have been carried out successfully (see Y. Gati [49]). For the numerical analysis of the particle approximation, we refer to M. Ben Alaya and B. Jourdain 9 .

\subsection{Blood flows}

Blood is a complex fluid consisting of a suspension of cells in plasma. These cells are mainly red blood cells or erythrocytes, white blood cells or leucocytes, and platelets. Red blood cells constitute $98 \%$ of the cells in suspension. These microstructures are mostly responsible for the non-Newtonian behavior of blood. A red blood cell is a biconcave disk of diameter $8.5 \mu \mathrm{m}$ and thickness $2.5 \mu \mathrm{m}$. It consists of a highly flexible membrane which is filled with a solution (haemoglobin). The ambient flow modifies the shape of the membrane. This phenomenon allows storage and release of energy in the microstructures, like for polymeric fluids. At low shear rates, red blood cells agglomerate into long structures called rouleaux.

It is observed that at high shear rates (like for pulsatile flow in healthy arteries, see for example J.F. Gerbeau, M. Vidrascu and P. Frey [50] or A. Quarteroni and L. Formaggia [106]), blood behaves essentially as a Newtonian fluid. At low shear rates (in arterioles, venules, recirculatory regions in aneurysms and stenoses), blood is a non-Newtonian fluid: it exhibits shear-thining, viscoelastic and thixotropic effects. This can be interpreted as follows: in flows with high shear rates, red blood cells cannot agglomerate, and the rheology is not influenced by the microstructures, while in flows with low shear rates, red blood cells agglomerate and this influences the rheology. Notice that we here discuss simple mechanical properties, neglecting important biochemical factors (like in clot formation for example).

In [102, 41, R.G. Owens and J. Fang propose a micro-macro model for blood, which is very similar to the model presented in Section 4 . This model applies in some sufficiently large flow domains, so that statistics on the configurations of red blood cells at each macroscopic point make sense. In other context, it may be important to consider each red blood cell as a separated entity like in the work [80] by A. Lefebvre and B. Maury.

Let us first suppose that the velocity field is given and homogeneous. The microscopic variables used to describe the microstructure (namely the red blood cells) are a vector $\boldsymbol{X}$ (similar to the end-to-end vector for polymeric fluids) and an integer $k \geq 1$ which measures 
the size of the aggregate the red blood cell belongs to. Consider then the non-negative function $\psi_{k}(t, \boldsymbol{X})$ such that $\psi_{k}(t, \boldsymbol{X}) d \boldsymbol{X}$ is the number of red blood cells (per unit volume of fluid) belonging to an aggregate of size $k$ having end-to-end vector between $\boldsymbol{X}$ and $\boldsymbol{X}+d \boldsymbol{X}$. We denote by $N_{j}=\frac{1}{j} \int \psi_{j}(t, \boldsymbol{X}) d \boldsymbol{X}$ the number of aggregates of $k$ red blood cells per unit volume.

The following Fokker-Planck equation rules the evolution of $\left(\psi_{k}(t, \boldsymbol{X})\right)_{k \geq 1}$ :

$$
\begin{aligned}
\frac{\partial \psi_{k}}{\partial t}= & -\operatorname{div} \boldsymbol{X}\left(\left(\nabla \boldsymbol{u} \boldsymbol{X}-\frac{2}{\zeta_{k}} \boldsymbol{F}(\boldsymbol{X})\right) \psi_{k}\right)+\frac{2 k T}{\zeta_{k}} \Delta_{\boldsymbol{X}} \psi_{k} \\
& +h_{k}(\dot{\gamma}) \psi_{k}^{e q}-g_{k}(\dot{\gamma}) \psi_{k} .
\end{aligned}
$$

In Equation (7.8,

$$
h_{k}(\dot{\gamma})=\frac{a(\dot{\gamma})}{2 N_{k}^{e q}} \sum_{i=1}^{k-1} N_{i} N_{k-i}+\frac{b(\dot{\gamma})}{N_{k}^{e q}} \sum_{j=1}^{\infty} N_{k+j}
$$

is an aggregation rate coefficient and

$$
g_{k}(\dot{\gamma})=\frac{b(\dot{\gamma})}{2}(k-1)+a(\dot{\gamma}) \sum_{j=1}^{\infty} N_{j}
$$

is a fragmentation rate coefficient. Both depend on the shear rate $\dot{\gamma}=\sqrt{\frac{1}{2} \dot{\gamma}: \dot{\gamma}}$ with $\dot{\gamma}=\nabla \boldsymbol{u}+\nabla \boldsymbol{u}^{T}$. At equilibrium (namely for zero shear rate: $\dot{\gamma}=0$ ), the number of aggregates of $k$ red blood cells per unit volume is $N_{k}^{e q}$. An analytical expression for $N_{k}^{e q}$ can be derived, in terms of $a(0), b(0)$ and the total number of red blood cells per unit volume $N_{0}$ (which is a conserved quantity). The function $\psi_{k}^{e q}=Z^{-1} \exp (-\Pi) k N_{k}^{e q}$ describes the statistics of the red blood cells at equilibrium ( $\Pi$ is the potential of the force $\boldsymbol{F}$ ). Notice that by integrating (7.8) with respect to $\boldsymbol{X}$ (and dividing by $k$ ), the following Smoluchowski equation on $\left(N_{k}(t)\right)_{k \geq 1}$ is obtained:

$$
\frac{d N_{k}}{d t}=h_{k}(\dot{\gamma}) N_{k}^{e q}-g_{k}(\dot{\gamma}) N_{k} .
$$

The parameters of the model are $N_{0}$, the friction coefficient $\zeta_{k}$ (which is typically chosen as $\zeta_{k}=k \zeta_{1}$ ) and the functions $a$ and $b$ which can be calibrated using experiments (see R.G. Owens and J. Fang [102, 41]).

In complex flows (for which $\nabla \boldsymbol{u}$ depends on the space variable $\boldsymbol{x}$ ), the functions $\psi_{k}$ also depend on $\boldsymbol{x}$ and the derivative $\frac{\partial}{\partial t}$ in $(\mathbf{7 . 8})$ is replaced by a convective derivative $\frac{\partial}{\partial t}+\boldsymbol{u} \cdot \nabla$. The micro model is coupled to the momentum equations through the Kramers expression for the extra stress tensor:

$$
\begin{gathered}
\boldsymbol{\tau}=\sum_{k=1}^{\infty} \boldsymbol{\tau}_{k} \\
\boldsymbol{\tau}_{k}(t, \boldsymbol{x})=\int \boldsymbol{F}(\boldsymbol{X}) \otimes \boldsymbol{X} \psi_{k}(t, \boldsymbol{x}, \boldsymbol{X}) d \boldsymbol{X}-k N_{k}(t, \boldsymbol{x}) k_{B} T \mathrm{Id} .
\end{gathered}
$$


Let us mention one modelling challenge: it is observed that the distribution of red blood cells is not uniform across a vessel (cell-depleted region near the vessel walls), and it is not clear how to account for this phenomenon in the micro-macro model. In the case of a Hookean force, it is possible to derive a macro-macro version of this model, which can then be further simplified (see R.G. Owens and J. Fang [102, 41]). Only this macro-macro version has been used so far in simulations for comparisons with experimental datas (see again R.G. Owens and J. Fang [102, 41]).

\section{References}

[1] M.P. Allen and D.J. Tildesley. Computer simulation of liquids. Oxford Science Publications, 1987.

[2] A. Ammar, B. Mokdad, F. Chinesta, and R. Keunings. A new family of solvers for some classes of multidimensional partial differential equations encountered in kinetic theory modeling of complex fluids. J. Non-Newtonian Fluid Mech., 139:153-176, 2006.

[3] A. Ammar, B. Mokdad, F. Chinesta, and R. Keunings. A new family of solvers for some classes of multidimensional partial differential equations encountered in kinetic theory modeling of complex, part II: Transient simulation using space-time separated representations. J. Non-Newtonian Fluid Mech., 144:98-121, 2007.

[4] C. Ané, S. Blachère, D. Chafaï, P. Fougères, I. Gentil, F. Malrieu, C. Roberto, and G. Scheffer. Sur les inégalités de Sobolev logarithmiques. SMF, 2000. In French.

[5] A. Arnold, P. Markowich, G. Toscani, and A. Unterreiter. On convex Sobolev inequalities and the rate of convergence to equilibrium for Fokker-Planck type equations. Comm. Part. Diff. Eq., 26:43-100, 2001.

[6] F.P.T. Baaijens. Mixed finite element methods for viscoelastic flow analysis: a review. J. Non-Newtonian Fluid Mech., 79:361-385, 1998.

[7] J.W. Barrett, C. Schwab, and E. Suli. Existence of global weak solutions for some polymeric flow models. Math. Models and Methods in Applied Sciences, 15(6):939-983, 2005.

[8] J.W. Barrett and E. Suli. Existence of global weak solutions to kinetic models for dilute polymers. Multiscale Model. Simul., 6(2):506-546, 2007.

[9] M. Ben Alaya and B. Jourdain. Probabilistic approximation of a nonlinear parabolic equation occuring in rheology. Journal of Applied Probability, 2007. To appear.

[10] D. Bernardin. Introduction à la rhéologie des fluides : approche macroscopique, 2003. Ecole de printemps, G.D.R. Matériaux vitreux, disponible à http://www.Imcp.jussieu.fr/lmcp/GDR-verres/html/Rheologi_1.pdf. In French. 
[11] R.B. Bird, R.C. Armstrong, and O. Hassager. Dynamics of polymeric liquids, volume 1. Wiley Interscience, 1987.

[12] R.B. Bird, C.F. Curtiss, R.C. Armstrong, and O. Hassager. Dynamics of polymeric liquids, volume 2. Wiley Interscience, 1987.

[13] R.B. Bird, P.J. Dotson, and N.L. Johnson. Polymer solution rheology based on a finitely extensible bead-spring chain model. J. Non-Newtonian Fluid Mech., 7:213235, 1980. Errata: J. Non-Newtonian Fluid Mech., 8:193 (1981).

[14] A. Bonito, Ph. Clément, and M. Picasso. Finite element analysis of a simplified stochastic Hookean dumbbells model arising from viscoelastic flows. M2AN Math. Model. Numer. Anal., 40(4):785-814, 2006.

[15] A. Bonito, Ph. Clément, and M. Picasso. Mathematical analysis of a stochastic simplified Hookean dumbbells model arising from viscoelastic flow. J. Evol. Equ., 6(3):381$398,2006$.

[16] J. Bonvin and M. Picasso. Variance reduction methods for CONNFFESSIT-like simulations. J. Non-Newtonian Fluid Mech., 84:191-215, 1999.

[17] J. Bonvin, M. Picasso, and R. Sternberg. GLS and EVSS methods for a three fields stokes problem arising from viscoelastic flows. Comp. Meth. Appl. Mech. Eng., 190(3893-3914), 2001.

[18] J.C. Bonvin. Numerical simulation of viscoelastic fluids with mesoscopic models. PhD thesis, Ecole Polytechnique Fédérale de Lausanne, 2000. Available at http://library.epfl.ch/theses/?nr=2249.

[19] M. Braack and A. Ern. A posteriori control of modeling errors and discretization errors. Multiscale Model. Simul., 1(2):221-238, 2003.

[20] H.-J. Bungartz and M. Griebel. Sparse grids. Acta Numer., 13:147-269, 2004.

[21] E. Cancès, I. Catto, and Y. Gati. Mathematical analysis of a nonlinear parabolic equation arising in the modelling of non-newtonian flows. SIAM J. Math. Anal., $37: 60-82,2005$.

[22] E. Cancès, I. Catto, Y. Gati, and C. Le Bris. A micro-macro model describing couette flows of concentrated suspensions. SIAM J. Multiscale Modeling and Simulation, 4:1041-1058, 2005.

[23] E. Cancès and C. Le Bris. Convergence to equilibrium of a multiscale model for suspensions. $D C D S-B, 6: 449-470,2006$.

[24] C. Chauvière. A new method for micro-macro simulations of viscoelastic flows. SIAM J. Sci. Comput., 23(6):2123-2140, 2002. 
[25] C. Chauvière and A. Lozinski. Simulation of dilute polymer solutions using a FokkerPlanck equation. Computers and fluids, 33(5-6):687-696, 2004.

[26] F. Comets and T. Meyre. Calcul stochastique et modèles de diffusion. Dunod, 2006. In French.

[27] P. Constantin. Nonlinear Fokker-Planck Navier-Stokes systems. Commun. Math. Sci., $3(4): 531-544,2005$.

[28] P. Constantin, C. Fefferman, A. Titi, and A. Zarnescu. Regularity of coupled twodimensional nonlinear Fokker-Planck and Navier-Stokes systems, 2006. Preprint available at http://jp.arxiv.org/abs/math/0605245.

[29] P. Constantin, I. Kevrekidis, and E.S. Titi. Asymptotic states of a Smoluchowski equation. Archive Rational Mech. Analysis, 174(3):365-384, 2004.

[30] P. Constantin, I. Kevrekidis, and E.S. Titi. Remarks on a Smoluchowski equation. Disc. and Cont. Dyn. Syst., 11(1):101-112, 2004.

[31] P. Constantin and N. Masmoudi. Global well posedness for a Smoluchowski equation coupled with Navier-Stokes equations in $2 \mathrm{~d}$. Technical report, New York University, 2007. Preprint available at http://www . math. nyu.edu/faculty/masmoudi/index.html.

[32] P. Degond, M. Lemou, and M. Picasso. Viscoelastic fluid models derived from kinetic equations for polymers. SIAM J. Appl. Math., 62(5):1501-1519, 2002.

[33] P. Delaunay, A. Lozinski, and R.G. Owens. Sparse tensor-product Fokker-Planckbased methods for nonlinear bead-spring chain models of dilute polymer solutions. CRM Proceedings and Lecture Notes, Volume 41, 2007.

[34] F. Devreux. Matière et désordre : polymères, gels, verres. Cours de l'Ecole Polytechnique, 2000. In French.

[35] M. Doi. Introduction to Polymer Physics. International Series of Monographs on Physics. Oxford University Press, 1996.

[36] M. Doi and S.F. Edwards. The Theory of Polymer Dynamics. International Series of Monographs on Physics. Oxford University Press, 1988.

[37] Q. Du, C. Liu, and P. Yu. FENE dumbbell model and its several linear and nonlinear closure approximations. SIAM Journal on Multiscale Modeling and Simulation, $4(3): 709-731,2005$.

[38] W. E, T. Li, and P.W. Zhang. Convergence of a stochastic method for the modeling of polymeric fluids. Acta Mathematicae Applicatae Sinica, English Series, 18(4):529-536, 2002. 
[39] W. E, T. Li, and P.W. Zhang. Well-posedness for the dumbbell model of polymeric fluids. Commun. Math. Phys., 248:409-427, 2004.

[40] A. Ern and T. Lelièvre. Adaptive models for polymeric fluid flow simulation. C. $R$. Acad. Sci. Paris, Ser. I, 344(7):473-476, 2007.

[41] J. Fang and R.G. Owens. Numerical simulations of pulsatile blood flow using a new constitutive model. Biorheology, 43:637-770, 2006.

[42] R. Fattal and R. Kupferman. Constitutive laws for the matrix-logarithm of the conformation tensor. J. Non-Newtonian Fluid Mech., 123:281-285, 2004.

[43] R. Fattal and R. Kupferman. Time-dependent simulation of viscoelastic flows at high Weissenberg number using the log-conformation representation. J. Non-Newtonian Fluid Mech., 126:23-37, 2005.

[44] E. Fernández-Cara, F. Guillén, and R.R. Ortega. Handbook of numerical analysis. Vol. 8: Solution of equations in $\mathbb{R}^{n}$ (Part 4). Techniques of scientific computing (Part 4). Numerical methods of fluids (Part 2)., chapter Mathematical modeling and analysis of viscoelastic fluids of the Oldroyd kind, pages 543-661. Amsterdam: North Holland/ Elsevier, 2002.

[45] G. Forest, Q. Wang, and R. Zhou. The flow-phase diagram of Doi-Hess theory for sheared nematic polymers II: finite shear rates. Rheol. Acta, 44(1):80-93, 2004.

[46] M. Fortin and A. Fortin. A new approach for the FEM simulation of viscoelastic flows. J. Non-Newtonian Fluid Mech., 32:295-310, 1989.

[47] D. Frenkel and B. Smit. Understanding molecular dynamics: from algorithms to applications. Academic Press, London, 2002.

[48] H. Gao and P. Klein. Numerical simulation of crack growth in an isotropic solid with randomized internal cohesive bonds. J. Mech. Phys. Solids, 46(2):187-218, 1998.

[49] Y. Gati. Modélisation mathématique et simulations numériques de fluides non newtoniens. PhD thesis, Ecole Nationale des Ponts et Chaussées, 2004. Available at http://pastel.paristech.org/883/01/these.pdf . In French.

[50] J.-F. Gerbeau, M. Vidrascu, and P. Frey. Fluid-structure interaction in blood flows on geometries coming from medical imaging. Computers and Structure, 83(2-3):155-165, 2005 .

[51] H. Giesekus. A simple constitutive equation for polymeric fluids based on the concept of deformation-dependent tensorial mobility. J. Non-Newtonian Fluid Mech., 11:69$109,1982$.

[52] R. Guénette and M. Fortin. A new mixed finite element method for computing viscoelastic flows. J. Non-Newtonian Fluid Mech., 60:27-52, 1999. 
[53] C. Guillopé and J.C. Saut. Existence results for the flow of viscoelastic fluids with a differential constitutive law. Nonlinear Analysis, Theory, Methods \& Appl., 15(9):849$869,1990$.

[54] C. Guillopé and J.C. Saut. Global existence and one-dimensional nonlinear stability of shearing motions of viscoelastic fluids of Oldroyd type. RAIRO Math. Model. Num. Anal., 24(3):369-401, 1990.

[55] E. Hairer, S.P. Norsett, and G. Wanner. Solving ordinary differential equations I. Springer, 1992.

[56] E. Hairer and G. Wanner. Solving ordinary differential equations II. Springer, 2002.

[57] P. Halin, G. Lielens, R. Keunings, and V. Legat. The Lagrangian particle method for macroscopic and micro-macro viscoelastic flow computations. J. Non-Newtonian Fluid Mech., 79:387-403, 1998.

[58] D.J. Higham. An algorithmic introduction to numerical simulation of stochastic differential equations. SIAM Review, 43(3):525-546, 2001.

[59] D. Hu and T. Lelièvre. New entropy estimates for the Oldroyd-B model, and related models, 2007. Available at http://arxiv.org/abs/math.NA/0703198, submitted to Commun. Math. Sci.

[60] M.A. Hulsen, R. Fattal, and R. Kupferman. Flow of viscoelastic fluids past a cylinder at high weissenberg number: stabilized simulations using matrix logarithms. Journal of Non-Newtonian Fluid Mechanics, 127(1):27-39, 2005.

[61] M.A. Hulsen, A.P.G. van Heel, and B.H.A.A. van den Brule. Simulation of viscoelastic flows using Brownian configuration fields. J. Non-Newtonian Fluid Mech., 70:79-101, 1997.

[62] P. Hébraud and F. Lequeux. Mode-coupling theory for the pasty rheology of soft glassy materials. Phys. Rev. Lett., 81:2934-2937, 1998.

[63] B. Jourdain, C. Le Bris, and T. Lelièvre. On a variance reduction technique for micromacro simulations of polymeric fluids. J. Non-Newtonian Fluid Mech., 122:91-106, 2004.

[64] B. Jourdain, C. Le Bris, and T. Lelièvre. An elementary argument regarding the longtime behaviour of the solution to a stochastic differential equation. Annals of Craiova University, Mathematics and Computer Science series, 32:1-9, 2005.

[65] B. Jourdain, C. Le Bris, T. Lelièvre, and F. Otto. Long-time asymptotics of a multiscale model for polymeric fluid flows. Archive for Rational Mechanics and Analysis, 181(1):97-148, 2006. 
[66] B. Jourdain and T. Lelièvre. Mathematical analysis of a stochastic differential equation arising in the micro-macro modelling of polymeric fluids. In I.M. Davies, N. Jacob, A. Truman, O. Hassan, K. Morgan, and N.P. Weatherill, editors, Probabilistic Methods in Fluids Proceedings of the Swansea 2002 Workshop, pages 205-223. World Scientific, 2003.

[67] B. Jourdain, T. Lelièvre, and C. Le Bris. Numerical analysis of micro-macro simulations of polymeric fluid flows: a simple case. Math. Models and Methods in Applied Sciences, 12(9):1205-1243, 2002.

[68] B. Jourdain, T. Lelièvre, and C. Le Bris. Existence of solution for a micro-macro model of polymeric fluid: the FENE model. Journal of Functional Analysis, 209:162-193, 2004 .

[69] I. Karatzas and S.E. Shreve. Brownian motion and stochastic calculus. Springer-Verlag, 1988.

[70] R. Keunings. Fundamentals of Computer Modeling for Polymer Processing, chapter Simulation of viscoelastic fluid flow, pages 402-470. Hanser, 1989.

[71] R. Keunings. A survey of computational rheology. In D.M. Binding et al., editor, Proc. 13th Int. Congr. on Rheology, pages 7-14. British Society of Rheology, 2000.

[72] R. Keunings. Micro-macro methods for the multiscale simulation of viscoelastic flows using molecular models of kinetic theory. In D.M. Binding and K. Walters, editors, Rheology Reviews 2004. British Society of Rheology, 2004.

[73] P.E. Kloeden and E. Platen. Numerical Solution of Stochastic Differential Equations, volume 23 of Applications of Mathematics. Springer, 1992.

[74] Y. Kwon. Finite element analysis of planar 4:1 contraction flow with the tensorlogarithmic formulation of differential constitutive equations. Korea-Australia Rheology Journal, 16(4):183-191, 2004.

[75] M. Laso and H.C. Öttinger. Calculation of viscoelastic flow using molecular models : The CONNFFESSIT approach. J. Non-Newtonian Fluid Mech., 47:1-20, 1993.

[76] M. Laso, M. Picasso, and H.C. Öttinger. Two-dimensional, time-dependent viscoelastic flow calculations using CONNFFESSIT. AIChE J., 43:877-892, 1997.

[77] C. Le Bris. Systèmes multiéchelles: modélisation et simulation, volume 47 of Mathématiques et Applications. Springer, 2005. In French.

[78] C. Le Bris and P.L. Lions. Renormalized solutions to some transport equations with partially $W^{1,1}$ velocities and applications. Annali di Matematica pura ed applicata, 183:97-130, 2004. 
[79] C. Le Bris and P.L. Lions. Existence and uniqueness of solutions to Fokker-Planck type equations with irregular coefficients. Comm. Part. Diff. Eq., 2007. To appear.

[80] A. Lefebvre and B. Maury. Apparent viscosity of a mixture of a newtonian fluid and interacting particles. Comptes Rendus Académie des sciences, Mécanique, 333(12):923933, 2005 .

[81] T. Lelièvre. Optimal error estimate for the CONNFFESSIT approach in a simple case. Computers and Fluids, 33:815-820, 2004.

[82] T. Lelièvre. Problèmes mathématiques et numériques posés par la simulation d'écoulement de fluides polymériques. $\mathrm{PhD}$ thesis, Ecole Nationale des Ponts et Chaussées, 2004. Available at http://cermics.enpc.fr/ lelievre/rapports/these.pdf. In French.

[83] T. Li, H. Zhang, and P.W. Zhang. Local existence for the dumbbell model of polymeric fluids. Comm. Part. Diff. Eq., 29(5-6):903-923, 2004.

[84] T. Li and P.W. Zhang. Convergence analysis of BCF method for Hookean dumbbell model with finite difference scheme. SIAM MMS, 5(1):205-234, 2006.

[85] T. Li and P.W. Zhang. Mathematical analysis of multi-scale models of complex fluid. Comm. Math. Sci., 5(1):1-51, 2007.

[86] F. Lin, C. Liu, and P.W. Zhang. On hydrodynamics of viscoelastic fluids. Comm. Pure Appl. Math., 58(11):1437-1471, 2005.

[87] F. Lin, C. Liu, and P.W. Zhang. On a micro-macro model for polymeric fluids near equilibrium. Comm. Pure Appl. Math., 60(6):838-866, 2007.

[88] P.L. Lions and N. Masmoudi. Global solutions for some Oldroyd models of nonnewtonian flows. Chin. Ann. Math., Ser. B, 21(2):131-146, 2000.

[89] P.L. Lions and N. Masmoudi. Global existence of weak solutions to micro-macro models. C. R. Math. Acad. Sci., 345(1):15-20, 2007.

[90] A.S. Lodge. Elastic Liquids. Academic Press, 1964.

[91] A. Lozinski. Spectral methods for kinetic theory models of viscoelastic fluids. PhD thesis, Ecole Polytechnique Fédérale de Lausanne, 2003. Available at http://library.epfl.ch/theses/?nr=2860.

[92] A. Lozinski and C. Chauvière. A fast solver for Fokker-Planck equation applied to viscoelastic flows calculations. J. Comp. Phys., 189(2):607-625, 2003.

[93] L. Machiels, Y. Maday, and A.T. Patera. Output bounds for reduced-order approximations of elliptic partial differential equations. Comput. Methods Appl. Mech. Engrg., 190(26-27):3413-3426, 2001. 
[94] F. Malrieu. Inégalités de Sobolev logarithmiques pour des problèmes d'évolution non linéaires. PhD thesis, Université Paul Sabatier, 2001.

[95] J.M. Marchal and M.J. Crochet. A new mixed finite element for calculating viscoelastic flows. J. Non-Newtonian Fluid Mech., 26:77-114, 1987.

[96] N. Masmoudi. Well posedness for the FENE dumbbell model of polymeric flows. Technical report, New York University, 2007. Preprint available at http://www . math .nyu.edu/faculty/masmoudi/index.html.

[97] T. Min, J.Y. Yoo, and H. Choi. Effect of spatial discretization schemes on numerical solutions of viscoelastic fluid flows. J. Non-Newtonian Fluid Mech., 100:27-47, 2001.

[98] J.T. Oden and S. Prudhomme. Estimation of modeling error in computational mechanics. J. Comput. Phys., 182:496-515, 2002.

[99] J.T. Oden and K.S. Vemaganti. Estimation of local modeling error and goal-oriented adaptive modeling of heterogeneous materials. i. error estimates and adaptive algorithms. J. Comput. Phys., 164:22-47, 2000.

[100] B. Oksendal. Stochastic differential equations. An introduction with applications. Springer, 2003.

[101] H.C. Öttinger. Stochastic Processes in Polymeric Fluids. Springer, 1995.

[102] R.G. Owens. A new microstructure-based constitutive model for human blood. $J$. Non-Newtonian Fluid Mech., 140:57-70, 2006.

[103] R.G. Owens and T.N. Phillips. Computational rheology. Imperial College Press / World Scientific, 2002.

[104] A. Peterlin. Hydrodynamics of macromolecules in a velocity field with longitudinal gradient. J. Polym. Sci. B, 4:287-291, 1966.

[105] N. Phan-Thien and R.I. Tanner. A new constitutive equation derived from network theory. J. Non-Newtonian Fluid Mech., 2:353-365, 1977.

[106] A. Quarteroni and L. Formaggia. Mathematical modelling and numerical simulation of the cardiovascular system., volume 12 of Handbook of Numerical Analysis, chapter 1, pages 3-127. Elsevier, 2004. G. Ciarlet Ed. N. Ayache guest Ed.

[107] S. Reese. Meso-macro modelling of fibre-reinforced rubber-like composites exhibiting large elastoplastic deformation. International Journal of Solids and Structures, 40(4):951-980, 2003.

[108] S. Reese. A micromechanically motivated material model for the thermo-viscoelastic material behaviour of rubber-like polymers. International Journal of Plasticity, 19(7):909-940, 2003. 
[109] M. Renardy. Local existence of solutions of the Dirichlet initial-boundary value problem for incompressible hypoelastic materials. SIAM J. Math. Anal., 21(6):1369-1385, 1990.

[110] M. Renardy. An existence theorem for model equations resulting from kinetic theories of polymer solutions. SIAM J. Math. Anal., 22:313-327, 1991.

[111] M. Renardy. Mathematical analysis of viscoelastic flows. SIAM, 2000.

[112] D. Revuz and M. Yor. Continuous martingales and Brownian motion. Springer-Verlag, 1994.

[113] L.C.G. Rogers and D. Williams. Diffusions, Markov Processes, and Martingales, Volume 1: Foundations. Cambridge University Press, 2000.

[114] L.C.G. Rogers and D. Williams. Diffusions, Markov Processes, and Martingales, Volume 2: Itô calculus. Cambridge University Press, 2000.

[115] D. Sandri. Non integrable extra stress tensor solution for a flow in a bounded domain of an Oldroyd fluid. Acta Mech., 135(1-2):95-99, 1999.

[116] D. Stroock and S.R.S. Varadhan. Multidimensional diffusion processes. Springer, 1979.

[117] J.K.C. Suen, Y.L. Joo, and R.C. Armstrong. Molecular orientation effects in viscoelasticity. Annu. Rev. Fluid Mech., 34:417-444, 2002.

[118] A.P.G. Van Heel. Simulation of viscoelastic fluids: from microscopic models to macroscopic complex flows. PhD thesis, Delft University of Technology, 2000.

[119] T. von Petersdorff and C. Schwab. Numerical solution of parabolic equations in high dimensions. M2AN Math. Model. Numer. Anal., 38(1):93-127, 2004.

[120] P. Wapperom, R. Keunings, and V. Legat. The backward-tracking lagrangian particle method for transient viscoelastic flows. J. Non-Newtonian Fluid Mech., 91:273-295, 2000 .

[121] H. Zhang and P.W. Zhang. A theoretical and numerical study for the rod-like model of a polymeric fluid. Journal of Computational Mathematics, 22(2):319-330, 2004.

[122] H. Zhang and P.W. Zhang. Local existence for the FENE-dumbbell model of polymeric fluids. Archive for Rational Mechanics and Analysis, 2:373-400, 2006.

[123] L. Zhang, H. Zhang, and P.W. Zhang. Global weak solutions for Hookean-dumbbell model of dilute polymers. Preprint, 2007. 


\section{Index}

blood, 87

Brownian Configuration Field, 81

Brownian motion, 30

Cauchy deformation tensor, 16

closure approximation, 43,61

complex flow, 8

CONNFFESSIT, 46, 57, 81

constitutive law, 6

control variate, 58,61

Deborah number, 45

differential model, [1]

Doi model, 85 ]

drift, 30

elasticity, [7]

elongational flow, 8

end-to-end vector, 20

energy estimate, 75 ]

entropy, 79

Euler-Maruyama scheme, 37

Euler-Milstein scheme, 37

explicit Euler scheme, 36

FENE model, 42,60

FENE-P model, 15, 43, 61]

Feynman-Kac Formula, 33

filtration, 28

Finger tensor, 16

Fokker-Planck equation, 24

forward Euler scheme, 36

free energy, 78

Galerkin Least Square, 72

Giesekus model, 14,

Hebraud-Lequeux model, 86

High Weissenberg Number Problem, 47.

[73]

homogeneous flow, 8,74

homogeneous fluid, 7

inf-sup condition, $7 \mathbf{7 2}$

integral model, 11

Itô integral, 31

Kramers chain model, 20

Kramers formula, 25

Lamé coefficients, 6

Langevin equation, 37

liquid crystals, 84

material functions, $\mathbf{7}$

Maxwell model, 12

memory function, 16

monomers, 17

Navier-Stokes equation, 7

Nernst-Einstein relation, 38

Newtonian fluid, [6]

normal stress, 8

Oldroyd-B model, 11, 13

open syphon effects, 9

Ornstein-Uhlenbeck process, 37

overshoot phenomenon, 9

Phan-Thien Tanner model, 15

polymer, 17

probability space, 28

red blood cell, 87

Reynolds number, 14, 45]

rheology, $\mathbf{7}$

rod-climbing effect, 9

shear flow, 75

shear viscosity, 8

shear-thining, 9

simple flow, 7

INRIA 
simple shear flow, 8

stochastic differential equation, 30

stochastic process, 29

Streamline Upwind Petrov-Galerkin, 72

strong convergence, 35

three-field system, 11

Upper Convected Maxwell model, 13

upper-convected derivative, 14

variance reduction, 29, 58, 61

viscoelasticity, 7

viscosity, [7]

weak convergence, 36

Weissenberg effect, 9

Weissenberg number, 14, 45]

Young modulus, 12

zero-shear rate viscosity, 9 


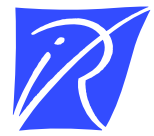

Unité de recherche INRIA Rocquencourt

Domaine de Voluceau - Rocquencourt - BP 105 - 78153 Le Chesnay Cedex (France)

Unité de recherche INRIA Futurs : Parc Club Orsay Université - ZAC des Vignes

4, rue Jacques Monod - 91893 ORSAY Cedex (France)

Unité de recherche INRIA Lorraine : LORIA, Technopôle de Nancy-Brabois - Campus scientifique

615, rue du Jardin Botanique - BP 101 - 54602 Villers-lès-Nancy Cedex (France)

Unité de recherche INRIA Rennes : IRISA, Campus universitaire de Beaulieu - 35042 Rennes Cedex (France)

Unité de recherche INRIA Rhône-Alpes : 655, avenue de l'Europe - 38334 Montbonnot Saint-Ismier (France)

Unité de recherche INRIA Sophia Antipolis : 2004, route des Lucioles - BP 93 - 06902 Sophia Antipolis Cedex (France)

INRIA - Domaine de Voluceau - Rocquencourt, BP 105 - 78153 Le Chesnay Cedex (France)

http://www.inria.fr

ISSN 0249-6399 